\title{
Random directed forest and the Brownian web
}

\author{
Rahul Roy, Kumarjit Saha and Anish Sarkar * \\ Indian Statistical Institute, New Delhi
}

\begin{abstract}
Consider the $d$ dimensional lattice $\mathbb{Z}^{d}$ where each vertex is open or closed with probability $p$ or $1-p$ respectively. An open vertex $\mathbf{u}:=$ $(\mathbf{u}(1), \mathbf{u}(2), \ldots, \mathbf{u}(d))$ is connected by an edge to another open vertex which has the minimum $L_{1}$ distance among all the open vertices $\mathbf{x}$ with $\mathbf{x}(d)>$ $\mathbf{u}(d)$. It is shown that this random graph is a tree almost surely for $d=2$ and 3 and it is an infinite collection of disjoint trees for $d \geq 4$. In addition, for $d=2$, we show that when properly scaled, the family of its paths converge in distribution to the Brownian web.
\end{abstract}

Key words: Markov chain, Random walk, Directed spanning forest, Brownian web.

AMS 2000 Subject Classification: 60D05.

\section{Introduction}

Let $\mathcal{P}$ be the points of a Poisson point process on $\mathbb{R}^{d}$ of intensity 1 . For each $\mathbf{x} \in \mathcal{P}$ let $h(\mathbf{x}) \in \mathcal{P}$ be the Poisson point in the half-space $\{\mathbf{u}: \mathbf{u}(d)>\mathbf{x}(d)\}$ which has the minimum Euclidean distance from $\mathbf{x}$, where $\mathbf{v}(j)$ denotes the $j$ th co-ordinate of $\mathbf{v} \in \mathbb{R}^{d}$. The directed spanning forest (DSF) is the random graph with vertex set $\mathcal{P}$ and edge set $\{\langle\mathbf{x}, h(\mathbf{x})\rangle: \mathbf{x} \in \mathcal{P}\}$. The study of the directed spanning forest (DSF) was initiated by Baccelli et al. [5]. Coupier et al. [8] proved that for $d=2$ the DSF is a tree almost surely. Ferrari et al. [12] also studied a directed random graph on a Poisson point process, however, the mechanism used to construct edges in that model incorporates more independence than is available in the DSF. They proved that their random graph is a connected tree in dimensions 2 and 3, and a forest in dimensions 4 and more.

*E-Mail: rahul, kumarjit10r, anish@isid.ac.in 
A similar construction, like the DSF arising from a Poisson point process, can be made from vertices of the integer lattice. Let $\left\{U_{\mathbf{v}}: \mathbf{v} \in \mathbb{Z}^{d}\right\}$ be a collection of i.i.d. uniform $(0,1)$ random variables. Fix $0<p<1$ and let $V:=\left\{\mathbf{v} \in \mathbb{Z}^{d}: U_{\mathbf{v}}<p\right\}$ be the set of open vertices of $\mathbb{Z}^{d}$. Given $\mathbf{u} \in \mathbb{Z}^{d}$, let $\mathbf{v} \in V$ be such that

1. $\mathbf{u}(d)<\mathbf{v}(d)$,

2. there does not exist any $\mathbf{w} \in V$ with $\mathbf{w}(d)>\mathbf{u}(d)$ such that $\|\mathbf{u}-\mathbf{w}\|_{1}<$ $\|\mathbf{u}-\mathbf{v}\|_{1}$, and

3. for all $\mathbf{w} \in V$ with $\mathbf{w}(d)>\mathbf{u}(d)$ and $\|\mathbf{u}-\mathbf{w}\|_{1}=\|\mathbf{u}-\mathbf{v}\|_{1}$ we have $U_{\mathbf{v}} \leq U_{\mathbf{w}}$.

Here and henceforth $\|\mathbf{u}\|_{1}$ denotes the $L_{1}$ norm of $\mathbf{u}$ on $\mathbb{R}^{d}$. Such a $\mathbf{v}$ is almost surely unique and clearly, is a function of $\mathbf{u}$ and $\mathbf{W}:=\left\{U_{\mathbf{w}}: \mathbf{w} \in \mathbb{Z}^{d}, \mathbf{w}(d)>\mathbf{u}(d)\right\}$. We denote it by $h(\mathbf{u}, \mathbf{W})$. We will drop the second argument in $h$ for the time being. Let $\langle\mathbf{u}, h(\mathbf{u})\rangle$ be the edge joining $\mathbf{u}$ and $h(\mathbf{u})$ and let $E$ denote the edge set given by,

$$
E:=\{\langle\mathbf{u}, h(\mathbf{u})\rangle: \mathbf{u} \in V\} .
$$

In this paper, we study the undirected random graph $G:=(V, E)$, which we will refer to as the discrete $D S F$ henceforth.

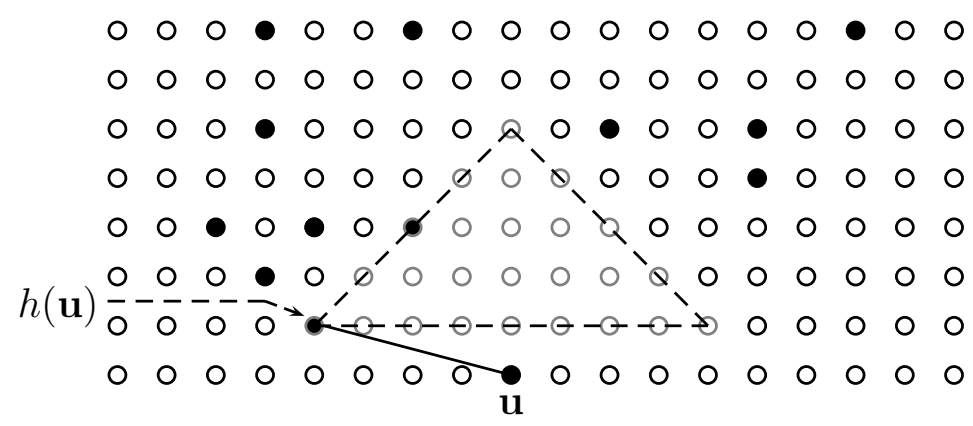

Figure 1: The construction of $h(\mathbf{u})$ from $\mathbf{u}$ on $\mathbb{Z}^{2}$. The shaded points are open, while the others are closed. Note that in order to get $h(\mathbf{u})$ from $\mathbf{u}$, we require information on the values of the uniform random variables of the gray vertices.

Similar models of random graphs are known in the physics literature as drainage networks (see Scheidegger [19]) and have been studied extensively (see RodríguezIturbe et al. [18]). Mathematically, for similar discrete processes but with a condition for constructing edges which allows more independence, the dichotomy in dimensions of having a single connected tree vis-a-vis a forest has been studied (see Gangopadhyay et al. [13, Athreya et al. [4]). The graph studied in [13] connected an open vertex $\mathbf{u}$ to the vertex $h(\mathbf{u})$ with $h(\mathbf{u})$ being the nearest open vertex in $\{\mathbf{w}: \mathbf{w}(d)=\mathbf{u}(d)+1\}$, with the vertex being chosen with uniform 
probability in case there are more than one nearest open vertex. This construction immediately leads to a Markovian analysis which is exploited in [13] to obtain the tree/forest dichotomy. However the DSF model considered here has to take care of a 'history' set arising from the paths constructed in the past. The Markovian structure is thus obtained through regeneration times. Moreover to obtain the dichotomy requires information on the tail of the distribution of the regeneration time which we do here through coupling and auxiliary results on renewal processes.

Our paper may also be viewed as an extension, albeit in the discrete setting, of the result of Coupier et al. [8] to any dimension. Our proof is different from that of [8]; while their argument is percolation theoretic and crucially depends on the planarity of $\mathbb{R}^{2}$, our argument exploits the Markovian structure of the DSF, thereby allowing us to extend the result to any dimension. The difficulty of carrying our analysis in the continuous model studied in [8] is that there is no obvious extension of regeneration time as considered here.

Theorem 1.1. For $d=2$ and $d=3$ the random graph $G$ is connected almost surely and consists of a single tree while for $d \geq 4$, it is a disconnected forest containing infinitely many distinct connected components, each connected component being an infinite tree almost surely.

Our second result in this paper is the convergence of the random graph $G$ for $d=2$, under a suitable diffusive scaling, to the Brownian web. The standard Brownian web originated in the work of Arratia [1], [2] as the scaling limit of the voter model on $\mathbb{Z}$. It arises naturally as the diffusive scaling limit of the coalescing simple random walk paths starting from every point on the space-time lattice. We can thus think of the Brownian web as a collection of one-dimensional coalescing Brownian motions starting from every point in the space time plane $\mathbb{R}^{2}$. Detailed analysis of the Brownian web was carried out in Tóth et al. [20]. Later Fontes et al. [11] introduced a framework in which the Brownian web is realized as a random variable taking values in a Polish space. We recall relevant details from Fontes et al. [11].

Let $\mathbb{R}_{c}^{2}$ denote the completion of the space time plane $\mathbb{R}^{2}$ with respect to the metric

$$
\rho\left(\left(x_{1}, t_{1}\right),\left(x_{2}, t_{2}\right)\right):=\left|\tanh \left(t_{1}\right)-\tanh \left(t_{2}\right)\right| \vee\left|\frac{\tanh \left(x_{1}\right)}{1+\left|t_{1}\right|}-\frac{\tanh \left(x_{2}\right)}{1+\left|t_{2}\right|}\right| .
$$

As a topological space $\mathbb{R}_{c}^{2}$ can be identified with the continuous image of $[-\infty, \infty]^{2}$ under a map that identifies the line $[-\infty, \infty] \times\{\infty\}$ with the point $(*, \infty)$, and the line $[-\infty, \infty] \times\{-\infty\}$ with the point $(*,-\infty)$. A path $\pi$ in $\mathbb{R}_{c}^{2}$ with starting time $\sigma_{\pi} \in[-\infty, \infty]$ is a mapping $\pi:\left[\sigma_{\pi}, \infty\right] \rightarrow[-\infty, \infty]$ such that $\pi(\infty)=*$ and, when $\sigma_{\pi}=-\infty, \pi(-\infty)=*$. Also $t \rightarrow(\pi(t), t)$ is a continuous map from $\left[\sigma_{\pi}, \infty\right]$ 
to $\left(\mathbb{R}_{c}^{2}, \rho\right)$. We then define $\Pi$ to be the space of all paths in $\mathbb{R}_{c}^{2}$ with all possible starting times in $[-\infty, \infty]$. The following metric, for $\pi_{1}, \pi_{2} \in \Pi$

$d_{\Pi}\left(\pi_{1}, \pi_{2}\right):=\left|\tanh \left(\sigma_{\pi_{1}}\right)-\tanh \left(\sigma_{\pi_{2}}\right)\right| \vee \sup _{t \geq \sigma_{\pi_{1}} \wedge \sigma_{\pi_{2}}}\left|\frac{\tanh \left(\pi_{1}\left(t \vee \sigma_{\pi_{1}}\right)\right)}{1+|t|}-\frac{\tanh \left(\pi_{2}\left(t \vee \sigma_{\pi_{2}}\right)\right)}{1+|t|}\right|$

makes $\Pi$ a complete, separable metric space. Convergence in this metric can be described as locally uniform convergence of paths as well as convergence of starting times. Let $\mathcal{H}$ be the space of compact subsets of $\left(\Pi, d_{\Pi}\right)$ equipped with the Hausdorff metric $d_{\mathcal{H}}$ given by,

$$
d_{\mathcal{H}}\left(K_{1}, K_{2}\right):=\sup _{\pi_{1} \in K_{1}} \inf _{\pi_{2} \in K_{2}} d_{\Pi}\left(\pi_{1}, \pi_{2}\right) \vee \sup _{\pi_{2} \in K_{2}} \inf _{\pi_{1} \in K_{1}} d_{\Pi}\left(\pi_{1}, \pi_{2}\right) .
$$

The space $\left(\mathcal{H}, d_{\mathcal{H}}\right)$ is a complete separable metric space. Let $B_{\mathcal{H}}$ be the Borel $\sigma$-algebra on the metric space $\left(\mathcal{H}, d_{\mathcal{H}}\right)$. The Brownian web $\mathcal{W}$ is an $\left(\mathcal{H}, B_{\mathcal{H}}\right)$ valued random variable.

Ferrari et al. [10] have shown that, for $d=2$, the random graph on the Poisson points introduced by [12], converges to a Brownian web under a suitable diffusive scaling. Coletti et al. [7] have a similar result for the discrete random graph studied in Gangopadhyay et al. [13]. Baccelli et al. [5] have shown that scaled paths of the successive ancestors in the DSF converges weakly to the Brownian motion and also conjectured that the scaling limit of the DSF is the Brownian web.

Our work here differs from that of [10] and [7] in that we need to obtain the Brownian web as a limit of a Markov process defined through regeneration times, while in the earlier work correlation inequalities like the FKG inequality could be used because every step of the paths constructing their model had i.i.d. increments. The method we employ requires us to control the size of the region surveyed to obtain the regeneration time of a process starting from a single vertex. Also using a martingale constructed via the joint regeneration times of processes starting from two distinct starting points we estimate the tail probability of the coalescing time. This method which we present here can be used in both [10] and [7] to obtain their results without invoking correlation inequalities. Also for the model considered by [5] and [8], if a suitable 'pseudo-regeneration time' of joint processes is defined and there is a control on the size of the region explored to obtain such pseudo-regeneration times, then our approach should yield the convergence to the Brownian web. In addition such pseudo-regeneration times should also yield the geometric structure of the DSF in dimensions 3 or more.

From a vertex $\mathbf{u} \in \mathbb{Z}^{2}$, taking the edges $\left.\left\{\left\langle h^{k-1}(\mathbf{u}), h^{k}(\mathbf{u})\right\rangle: k \geq 1\right)\right\}$ (with $h^{0}(\mathbf{u}):=\mathbf{u}$ and $\left.h^{k}(\mathbf{u}):=h\left(h^{k-1}(\mathbf{u})\right)\right)$ to be straight line segments we parametrize the path formed by these edges as the piecewise linear function $\pi^{\mathbf{u}}:[\mathbf{u}(2), \infty) \rightarrow \mathbb{R}$ 
such that $\pi^{\mathbf{u}}\left(h^{k}(\mathbf{u})(2)\right):=h^{k}(\mathbf{u})(1)$ for every $k \geq 0$ and $\pi^{\mathbf{u}}(t)$ is linear in the interval $\left[h^{k}(\mathbf{u})(2), h^{k+1}(\mathbf{u})(2)\right]$. Define $\mathcal{X}:=\left\{\pi^{\mathbf{u}}: \mathbf{u} \in V\right\}$. For given $\gamma, \sigma>0$, a path $\pi$ with starting time $\sigma_{\pi}$ and for each $n \geq 1$, the scaled path $\pi_{n}(\gamma, \sigma)$ : $\left[\sigma_{\pi} / n^{2} \gamma, \infty\right] \rightarrow[-\infty, \infty]$ is given by $\pi_{n}(\gamma, \sigma)(t):=\pi\left(n^{2} \gamma t\right) / n \sigma$. Thus, the scaled path $\pi_{n}(\gamma, \sigma)$ has the starting time $\sigma_{\pi_{n}(\gamma, \sigma)}=\sigma_{\pi} / n^{2} \gamma$. For each $n \geq 1$, let $\mathcal{X}_{n}(\gamma, \sigma):=\left\{\pi_{n}^{\mathbf{u}}(\gamma, \sigma): \mathbf{u} \in V\right\}$ be the collection of the scaled paths. The closure $\overline{\mathcal{X}}_{n}(\gamma, \sigma)$ of $\mathcal{X}_{n}(\gamma, \sigma)$ in $\left(\Pi, d_{\Pi}\right)$ is a $\left(\mathcal{H}, \mathcal{B}_{\mathcal{H}}\right)$ valued random variable. We have

Theorem 1.2. There exist $\sigma:=\sigma(p)$ and $\gamma:=\gamma(p)$ such that as $n \rightarrow \infty, \overline{\mathcal{X}}_{n}(\gamma, \sigma)$ converges weakly to the standard Brownian $W e b \mathcal{W}$ as $\left(\mathcal{H}, \mathcal{B}_{\mathcal{H}}\right)$ valued random variables.

Remark 1.3. The scaling property of the Brownian web yields $\overline{\mathcal{X}}_{n}\left(1, \sigma^{\prime}\right) \Rightarrow \mathcal{W}$ as $n \rightarrow \infty$ for $\sigma^{\prime}:=\sigma / \sqrt{\gamma}$.

For the proof of Theorem 1.1 we obtain a Markovian structure in our model and define suitable stopping times for this Markov process. From these stopping times the process regenerates which allows us to phrase the problem as a question of recurrence or transience of the Markov chain. This we do by obtaining a martingale for $d=2$, using a Lyapunov function technique for $d=3$ and a suitable coupling with a random walk with independent steps for $d=4$.

The martingale obtained for $d=2$ and the fact that the distributions of the stopping times have exponentially decaying tails are used to prove Theorem 1.2 ,

Finally, although our results are obtained for the random graph constructed by connecting edges between $L_{1}$ nearest open vertices, they should also hold for the model constructed with the $L_{2}$ metric (see Remark 2.5 for more details).

The paper is structured as follows - in the next section we construct the paths of the graph $G$ starting from $k$ distinct vertices and obtain some properties of these paths. In Section 3, we derive the martingale (for $d=2$ ) and also provide a method of approximation of the paths by independent processes, which is used later to prove Theorem 1.1 and Theorem 1.2, In Section 4 we prove Theorem 1.1 and in Section 5, we prove Theorem 1.2.

\section{Construction of the process}

We first detail a construction of the graph $G$ which is needed to bring out a Markovian structure. Later we obtain a martingale for $d=2$ which is used in the next two sections. Before proceeding further we fix some notation: for $\mathbf{u} \in \mathbb{Z}^{d}$ and $r \in \mathbb{Z}$, let $\mathbb{H}(r):=\left\{\mathbf{w} \in \mathbb{Z}^{d}: \mathbf{w}(d) \leq r\right\}$ be the half-space and, for $r>0$, let $S^{+}(\mathbf{u}, r):=\left\{\mathbf{w} \in \mathbb{Z}^{d}:\|\mathbf{u}-\mathbf{w}\|_{1} \leq r, \mathbf{w}(d)>\mathbf{u}(d)\right\}$ be the upper part of closed $L_{1}$ ball at $\mathbf{u}$ having radius $r$. As a convention we take $S^{+}(\mathbf{u}, 0):=\emptyset$. 
From $k(k \geq 1)$ vertices $\mathbf{u}^{1}, \ldots, \mathbf{u}^{k} \in \mathbb{Z}^{d}$ with $\mathbf{u}^{1}(d)=\cdots=\mathbf{u}^{k}(d)$, we obtain the vertices $\left\{h^{n}\left(\mathbf{u}^{i}\right): n \geq 0,1 \leq i \leq k\right\}$ as a stochastic process. Note here that the construction described below does not require the vertices $\mathbf{u}^{1}, \ldots, \mathbf{u}^{k}$ to be open. The vertices with the smallest $d$ th co-ordinate are allowed to move, while the others stay put (see Figure 2 and 3). Each of these vertices explores a region in the half space 'above' it to obtain the vertex to which it moves. During this exploration a vertex may encounter regions which have been already explored by other vertices earlier. While the information for the region explored earlier is known, the information about the freshly explored region is new and is obtained during the exploration process of the vertices which are moving at that time. The region which has been explored till the $n$th move of the entire process and which is needed for the $n+1$ th move is called the history region and the information of the uniform random variables in the history region constitutes the history. Formally, let

(i) $g_{0}\left(\mathbf{u}^{i}\right):=\mathbf{u}^{i}$ for all $1 \leq i \leq k$ and $r_{0}:=\mathbf{u}^{1}(d)$;

(ii) $W_{0}^{\text {move }}:=\left\{\mathbf{u}^{1}, \ldots, \mathbf{u}^{k}\right\}$ and $W_{0}^{\text {stay }}:=\emptyset$;

(iii) $\Delta_{0}=\Delta_{0}\left(\mathbf{u}^{1}, \ldots, \mathbf{u}^{k}\right):=\emptyset$ and $\Psi_{0}: \emptyset \rightarrow[0,1]$ the empty function (see [14]).

Having obtained $g_{n}\left(\mathbf{u}^{i}\right), r_{n}, W_{n}^{\text {move }}, W_{n}^{\text {stay }}, \Delta_{n}$ and $\Psi_{n}$, for $1 \leq i \leq k$, we set

(i) $g_{n+1}(\mathbf{u}):=h\left(g_{n}(\mathbf{u})\right)$ for all $g_{n}(\mathbf{u}) \in W_{n}^{\text {move }}$ and $g_{n+1}(\mathbf{v}):=g_{n}(\mathbf{v})$ for all $g_{n}(\mathbf{v}) \in W_{n}^{\text {stay }}, r_{n+1}:=\min \left\{g_{n+1}\left(\mathbf{u}^{i}\right)(d): 1 \leq i \leq k\right\} ;$

(ii) $W_{n+1}^{\text {move }}:=\left\{g_{n+1}(\mathbf{w}): \mathbf{w} \in\left\{\mathbf{u}^{1}, \ldots, \mathbf{u}^{k}\right\}, g_{n+1}(\mathbf{w})(d)=r_{n+1}\right\}$ and $W_{n+1}^{\text {stay }}:=$ $\left\{g_{n+1}\left(\mathbf{u}^{1}\right), \ldots, g_{n+1}\left(\mathbf{u}^{k}\right)\right\} \backslash W_{n+1}^{\text {move }}$

(iii) $\Delta_{n+1}=\Delta_{n+1}\left(\mathbf{u}^{1}, \ldots, \mathbf{u}^{k}\right):=\left(\Delta_{n} \cup \cup_{\mathbf{u} \in W_{n}^{\text {move }}} S^{+}\left(\mathbf{u},\|h(\mathbf{u})-\mathbf{u}\|_{1}\right)\right) \backslash \mathbb{H}\left(r_{n+1}\right)$ and $\Psi_{n+1}: \Delta_{n+1} \rightarrow[0,1]$ is a map given by $\Psi_{n+1}(\mathbf{w}):=U_{\mathbf{w}}$ for $\mathbf{w} \in \Delta_{n+1}$, with $\Psi_{n+1}:=\Psi_{0}$, the empty function, when $\Delta_{n+1}=\emptyset$.

Let $\Delta \subseteq \mathbb{Z}^{d}$ be a finite union of $d$-dimensional tetrahedrons, with each tetrahedron in $\Delta$ having a $(d-1)$-dimensional tetrahedron as a base on the hyperplane $Q_{r}:=\left\{\mathbf{w} \in \mathbb{Z}^{d}: \mathbf{w}(d)=r\right\}$ for some $r \in \mathbb{Z}$. In other words, we have $\Delta=$ $\cup_{i=1}^{p} S^{+}\left(\mathbf{w}^{i}, t_{i}\right)$ for some $p \geq 1$ and $\mathbf{w}^{i} \in Q_{r}, t_{i} \geq 0$ for $1 \leq i \leq p$. We denote this class of subsets by $\mathcal{D}_{r}$. Further, for any $\Delta=\cup_{i=1}^{p} S^{+}\left(\mathbf{w}^{i}, t_{i}\right) \in \mathcal{D}_{r}$, let $\Psi: \Delta \rightarrow[0,1]$ be a mapping such that $\Psi(\mathbf{w}) \geq p$ for all $\mathbf{w} \in \Delta^{0}=\cup_{i=1}^{p} S^{+}\left(\mathbf{w}^{i}, t_{i}-1\right)$, with $\Psi$ being the empty function when $\Delta=\emptyset$. Let

$\mathfrak{S}^{(k)}:=\left\{\mathfrak{s}=\left(\mathbf{v}^{1}, \ldots, \mathbf{v}^{k}, \Delta, \Psi\right): \mathbf{v}^{i} \in \mathbb{Z}^{d}\right.$ for $i=1, \ldots, k, \Delta \in \mathcal{D}_{r}$ for $r:=\min \left\{\mathbf{v}^{1}(d), \ldots, \mathbf{v}^{k}(d)\right\}, \mathbf{v}^{i} \in \Delta \backslash \Delta^{0}$ for all $\mathbf{v}^{i}$ with $\mathbf{v}^{i}(d)>r$ and $\Psi$ satisfies the conditions above $\}$. 

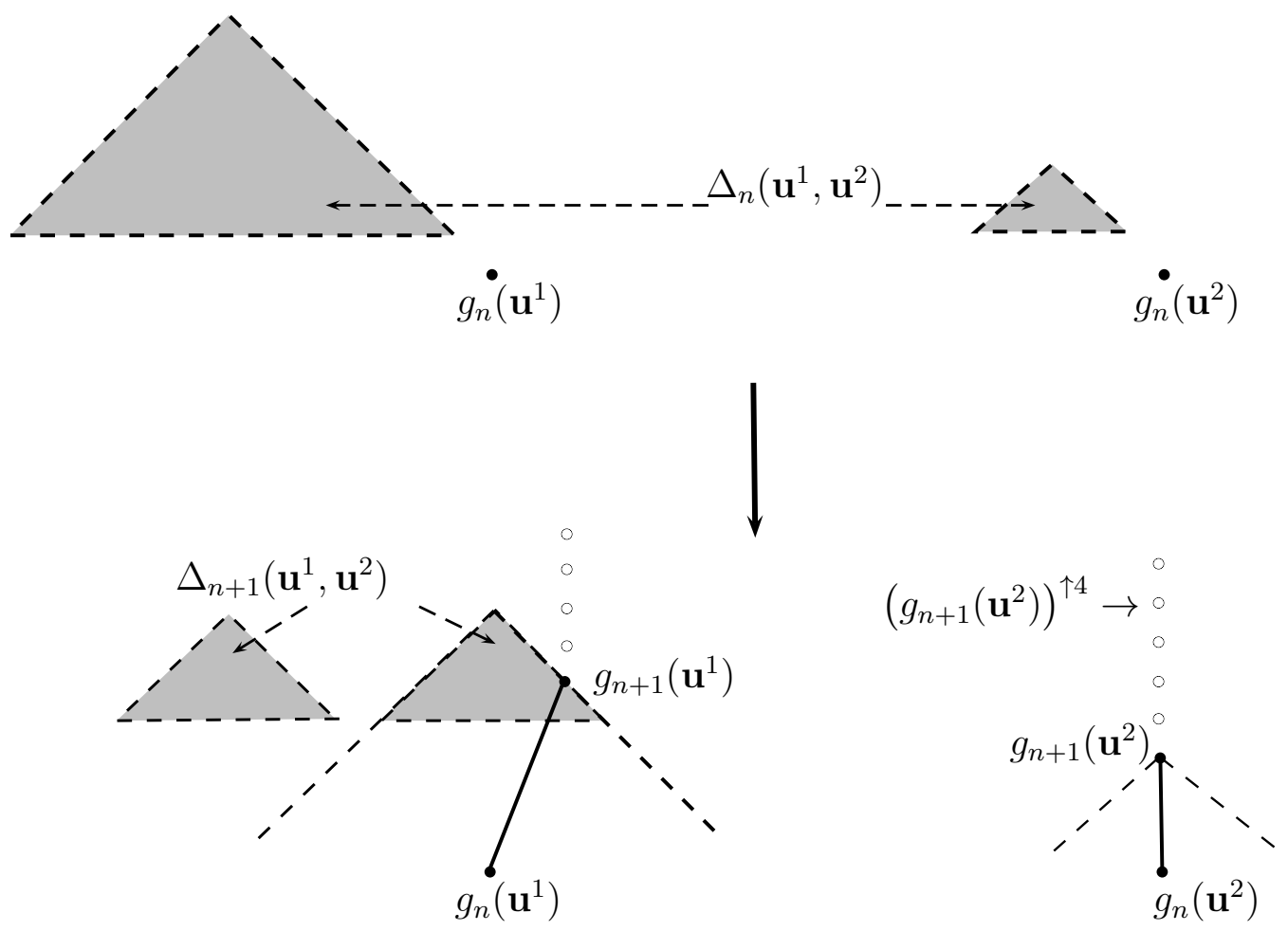

Figure 2: The vertices $g_{n+1}\left(\mathbf{u}^{1}\right), g_{n+1}\left(\mathbf{u}^{2}\right)$ and the history set $\Delta_{n+1}\left(\mathbf{u}^{1}, \mathbf{u}^{2}\right)$ when $W_{n}^{\text {move }}=\left\{g_{n}\left(\mathbf{u}^{1}\right), g_{n}\left(\mathbf{u}^{2}\right)\right\}, W_{n}^{\text {stay }}=\emptyset$. Note the vertices above $g_{n+1}\left(\mathbf{u}^{1}\right)$ and $g_{n+1}\left(\mathbf{u}^{2}\right)$ are unexplored.

Remark 2.1. We have the following observations:

(a) From the definition of the history region $\Delta_{n}$, formed at the $n$th step, is either empty or an element of $\mathcal{D}$ with the bases of tetrahedrons being contained in $Q_{r_{n}+1}$.

(b) Clearly, by definition of $r_{n}, W_{n}^{\text {move }} \subseteq Q_{r_{n}}$.

(c) From the definition of $\Delta_{n}$, all vertices in the set $\Xi_{n}:=\mathbb{Z}^{d} \backslash\left(\Delta_{n} \cup \mathbb{H}\left(r_{n}\right)\right)$ are unexplored until the $n+1$ th step, for each $n \geq 0$.

We now obtain the Markov process implicit in our construction. For each $n \geq 0$, set $\mathcal{Z}_{n}^{(k)}:=\left(g_{n}\left(\mathbf{u}^{1}\right), \ldots, g_{n}\left(\mathbf{u}^{k}\right), \Delta_{n}, \Psi_{n}\right)$. Clearly, $\mathcal{Z}_{n}^{(k)} \in \mathfrak{S}^{(k)}$. Let $\mathbf{Y}:=\left\{V_{\mathbf{w}}: \mathbf{w} \in\right.$ $\left.\mathbb{Z}^{d}, \mathbf{w}(d)>0\right\}$ be an independent collection of i.i.d. uniform $[0,1]$-valued random variables. For any $n \geq 1$, suppose $\mathcal{Z}_{n}^{(k)}=\mathfrak{s}\left(=\left(\mathbf{v}_{\tilde{Y}}^{1}, \ldots, \mathbf{v}^{k}, \Delta, \Psi\right)\right)$ for some $\mathfrak{s} \in$ $\mathfrak{S}^{(k)}$. We define the collection of random variables $\tilde{\mathbf{Y}}:=\left\{\tilde{V}_{\mathbf{w}}: \mathbf{w} \in \mathbb{Z}^{d}, \mathbf{w}(d)>r\right\}$ 


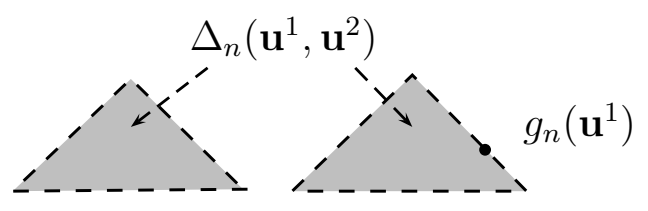

$$
. g_{n}\left(\mathbf{u}^{2}\right)
$$

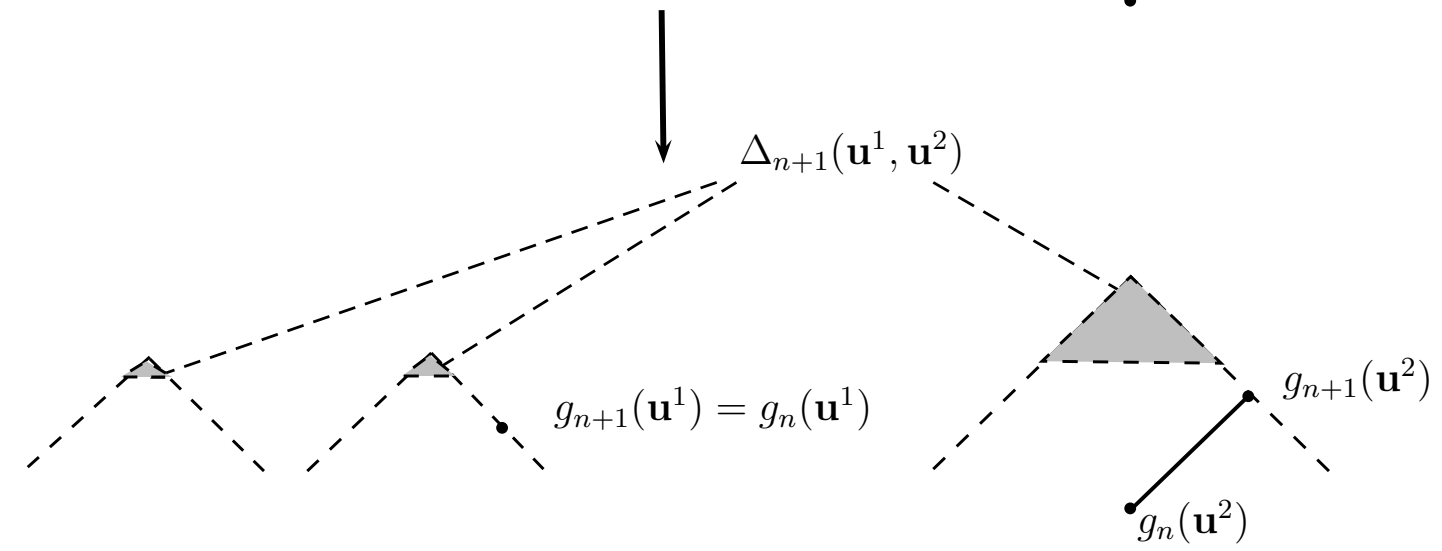

Figure 3: The vertices $g_{n+1}\left(\mathbf{u}^{1}\right), g_{n+1}\left(\mathbf{u}^{2}\right)$ and the history region $\Delta_{n+1}\left(\mathbf{u}^{1}, \mathbf{u}^{2}\right)$ when $W_{n}^{\text {move }}=\left\{g_{n}\left(\mathbf{u}^{2}\right)\right\}, W_{n}^{\text {stay }}=\left\{g_{n}\left(\mathbf{u}^{1}\right)\right\}$.

for $r=\min \left\{\mathbf{v}^{1}(d), \ldots, \mathbf{v}^{k}(d)\right\}$ as follows:

$$
\tilde{V}_{\mathbf{w}}:= \begin{cases}\Psi(\mathbf{w}) & \text { if } \mathbf{w} \in \Delta ; \\ V_{\mathbf{w}^{\prime}} & \text { if } \mathbf{w} \notin \Delta, \mathbf{w}(j)=\mathbf{w}^{\prime}(j), j \neq d \text { and } \mathbf{w}(d)=\mathbf{w}^{\prime}(d)+r .\end{cases}
$$

The above definition implies that $\tilde{\mathbf{Y}}$ is a function of $\mathbf{Y}$ and $\mathfrak{s}$, say $\tilde{\mathbf{Y}}=f(\mathbf{Y}, \mathfrak{s})$ where $f$ is a function from $[0,1]^{\mathbb{Z}^{d} \backslash \mathbb{H}(0)} \times \mathfrak{S}^{(k)}$ to $[0,1]^{\mathbb{Z}^{d} \backslash \mathbb{H}(r)}$. From the above definition and the fact that the vertices in $\Xi_{n}=\mathbb{Z}^{d} \backslash(\Delta \cup \mathbb{H}(r))$ are unexplored, and hence can be replaced by another set of i.i.d. uniform random variables, for the family $\mathbf{X}:=\left\{U_{\mathbf{w}}: \mathbf{w} \in \mathbb{Z}^{d}, \mathbf{w}(d)>r\right\}$, we have

$$
\mathbf{X} \mid \mathcal{Z}_{n}^{(k)} \stackrel{d}{=} f\left(\mathbf{Y}, \mathcal{Z}_{n}^{(k)}\right) .
$$

From the definition of the process, we obtain that $g_{n+1}\left(\mathbf{u}^{1}\right), \ldots, g_{n+1}\left(\mathbf{u}^{k}\right), \Delta_{n+1}$ and $\Psi_{n+1}$ is a function of $\mathcal{Z}_{n}^{(k)}=\left(g_{n}\left(\mathbf{u}^{1}\right), \ldots, g_{n}\left(\mathbf{u}^{k}\right), \Delta_{n}, \Psi_{n}\right)$ and $\mathbf{X}$, i.e.,

$$
\mathcal{Z}_{n+1}^{(k)}=f_{1}\left(\mathcal{Z}_{n}^{(k)}, \mathbf{X}\right)
$$

where $f_{1}$ is a function on $\mathfrak{S}^{(k)} \times[0,1]^{\mathbb{Z}^{d} \backslash \mathbb{H}\left(r_{n}\right)} \rightarrow \mathfrak{S}^{(k)}$. Therefore, from the above observation, the conditional distribution of $\mathcal{Z}_{n+1}^{(k)}$, given $\left\{\mathcal{Z}_{j}^{(k)}: 0 \leq j \leq n\right\}$, is the same as that of $f_{1}\left(\mathcal{Z}_{n}^{(k)}, f\left(\mathbf{Y}, \mathcal{Z}_{n}^{(k)}\right)\right)$. Hence, the process $\left\{\mathcal{Z}_{n}^{(k)}: n \geq 1\right\}$ admits a random mapping representation, which proves the Markov property (see, for example, Levin et al. [16]). 
Proposition 2.2. The process $\left\{\mathcal{Z}_{n}^{(k)}=\left(g_{n}\left(\mathbf{u}^{1}\right), g_{n}\left(\mathbf{u}^{2}\right), \ldots, g_{n}\left(\mathbf{u}^{k}\right), \Delta_{n}, \Psi_{n}\right): n \geq\right.$ 0\} is Markov with state space $\mathfrak{S}^{(k)}$.

For the remainder of this section we fix $\mathbf{u}^{1}, \ldots, \mathbf{u}^{k}$ with $\mathbf{u}^{1}(d)=\cdots=\mathbf{u}^{k}(d)$. Set $\tau_{0}=\tau\left(\mathbf{u}^{1}, \ldots, \mathbf{u}^{k}\right):=0$ and, for $l \geq 1$, define

$$
\begin{aligned}
\tau_{l} & =\tau_{l}\left(\mathbf{u}^{1}, \ldots, \mathbf{u}^{k}\right):=\inf \left\{n>\tau_{l-1}: \Delta_{n}=\emptyset\right\} \\
\sigma_{l} & =\sigma_{l}\left(\mathbf{u}^{1}, \ldots, \mathbf{u}^{k}\right):=\tau_{l}\left(\mathbf{u}^{1}, \ldots, \mathbf{u}^{k}\right)-\tau_{l-1}\left(\mathbf{u}^{1}, \ldots, \mathbf{u}^{k}\right) .
\end{aligned}
$$

We call $\tau_{l}$ the step at which the $l$ th simultaneous regeneration of $k$ joint paths occurs. We note here that $\tau_{l}$ denotes the number of steps (in the above construction) required for the joint process to regenerate (i.e., to reach a state of empty history for the $l$ th time) and $\sigma_{l}$ denotes the total number of steps (again in the above construction) between the $l-1$ th and $l$ th simultaneous regeneration of $k$ joint paths. This is not the same as the time (measured as the distance in the $d$ th co-ordinate) for regeneration, which we later denote by $T_{l}$ (see Figure 4). Also at each regeneration step $\tau_{l}$, the paths must be at the same level in terms of their $d$ th co-ordinate, i.e., $g_{\tau_{l}}\left(\mathbf{u}^{1}\right)(d)=\cdots=g_{\tau_{l}}\left(\mathbf{u}^{k}\right)(d)$.

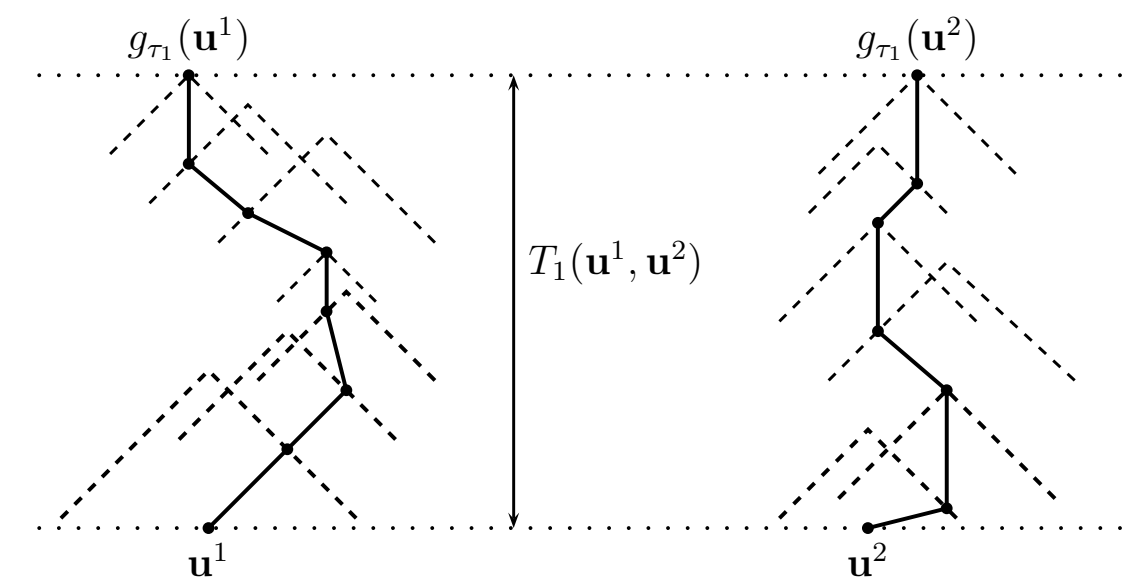

Figure 4: At regeneration step $\tau_{1}\left(\mathbf{u}^{1}, \mathbf{u}^{2}\right)$ of the process $g_{\tau_{1}}\left(\mathbf{u}^{1}\right)(d)=g_{\tau_{1}}\left(\mathbf{u}^{2}\right)(d)$ and $\Delta_{\tau_{1}}=\emptyset$

Our first task is to show that the Markov process, defined in Proposition 2.2, regenerates almost surely. In fact, we prove the much stronger statement that the number of inter-regeneration steps has exponentially decaying tail probabilities.

Proposition 2.3. For any $l \geq 1$ and $\mathbf{u}^{1}, \ldots, \mathbf{u}^{k} \in \mathbb{Z}^{d}$ with $\mathbf{u}^{1}(d)=\cdots=\mathbf{u}^{k}(d)$, we have

$$
\mathbb{P}\left(\sigma_{l} \geq n\right) \leq C_{1}^{(k)} \exp \left(-C_{2}^{(k)} n\right)
$$

for all $n \geq 1$, where $C_{1}^{(k)}$ and $C_{2}^{(k)}$ are positive constants, depending on $k$, but not on $l, n$ or $\mathbf{u}^{1}, \ldots, \mathbf{u}^{k}$. 
Since $\tau_{l}<\infty$ almost surely, we obtain that

Corollary 2.3.1. For $\mathbf{u}^{1}, \ldots, \mathbf{u}^{k}$ as above, the process

$$
\left\{\left(g_{\tau_{l}}\left(\mathbf{u}^{1}\right), \ldots, g_{\tau_{l}}\left(\mathbf{u}^{k}\right)\right): l \geq 0\right\} \text { is a Markov chain on }\left(\mathbb{Z}^{d}\right)^{k} \text {. }
$$

For $\mathbf{w} \in \mathbb{Z}^{d}$, let $(\mathbf{w})^{\uparrow m}$ be defined by

$$
(\mathbf{w})^{\uparrow m}(j):= \begin{cases}\mathbf{w}(j) & \text { for } 1 \leq j \leq d-1, \\ m+\mathbf{w}(d) & \text { for } j=d .\end{cases}
$$

the vertex w. Note

if $\mathbf{w} \in \mathbb{Z}^{d}, \mathbf{v} \in V$ are such that $\mathbf{v}(d)>\mathbf{w}(d)$, then $\|h(\mathbf{w})-\mathbf{w}\|_{1} \leq\|\mathbf{v}-\mathbf{w}\|_{1}$.

The main idea behind regeneration is contained in the above simple observation and the following Proposition 2.4.

Proposition 2.4. For $\mathbf{u}^{1}, \ldots, \mathbf{u}^{k}$ as in the construction of the process with the history region $\Delta_{n}$ and the quantity $r_{n}$ as described there, we have, for any $n \geq$ $0, m \geq 1$ and $1 \leq i \leq k$, the vertex $\left(g_{n}\left(\mathbf{u}^{i}\right)\right)^{\uparrow m} \notin \Delta_{n} \cup \mathbb{H}\left(r_{n}\right)$.

Proof : Fix $n \geq 0, m \geq 1$ and $1 \leq i \leq k$. We have $\left(g_{n}\left(\mathbf{u}^{i}\right)\right)^{\uparrow m}(d)=g_{n}\left(\mathbf{u}^{i}\right)(d)+$ $m>g_{n}\left(\mathbf{u}^{i}\right)(d) \geq r_{n}$, so that $\left(g_{n}\left(\mathbf{u}^{i}\right)\right)^{\uparrow m} \notin \mathbb{H}\left(r_{n}\right)$. It is enough to show $\left(g_{n}\left(\mathbf{u}^{i}\right)\right)^{\uparrow m} \notin$ $\cup_{j=0}^{n} \Delta_{j}$. First $\left(g_{n}\left(\mathbf{u}^{i}\right)\right)^{\uparrow m} \notin \Delta_{0}$. So take $n \geq 1$ and inductively assume that $\left(g_{n}\left(\mathbf{u}^{i}\right)\right)^{\uparrow m} \notin \Delta_{j}$ for some $0 \leq j \leq n-1$. Let $\mathbf{w} \in W_{j}^{\text {move }}$. Since the vertices with the smallest $d$ th co-ordinate are in $W_{j}^{\text {move }}$ and, in the next step their $d$ th co-ordinate increases by at least one unit, we have $\mathbf{w}(d)=r_{j}<r_{n} \leq g_{n}\left(\mathbf{u}^{i}\right)(d)$. Since $g_{n}\left(\mathbf{u}^{i}\right) \in V$, clearly $\|h(\mathbf{w})-\mathbf{w}\|_{1} \leq\left\|g_{n}\left(\mathbf{u}^{i}\right)-\mathbf{w}\right\|_{1}$. Further, we have $\left\|\left(g_{n}\left(\mathbf{u}^{i}\right)\right)^{\uparrow m}-\mathbf{w}\right\|_{1}=m+\left\|g_{n}\left(\mathbf{u}^{i}\right)-\mathbf{w}\right\|_{1}>\left\|g_{n}\left(\mathbf{u}^{i}\right)-\mathbf{w}\right\|_{1}$ and hence $\left(g_{n}\left(\mathbf{u}^{i}\right)\right)^{\uparrow m} \notin$ $S^{+}\left(\mathbf{w},\|h(\mathbf{w})-\mathbf{w}\|_{1}\right)$. Thus, $\left(g_{n}\left(\mathbf{u}^{i}\right)\right)^{\uparrow m} \notin \Delta_{j+1}$.

In order to prove Proposition 2.3, we define a random variable $L_{n}$ which represents the height of the history region $\Delta_{n}$, measured along the $d$ th co-ordinate from the lowest vertex among $g_{n}\left(\mathbf{u}^{1}\right), \ldots, g_{n}\left(\mathbf{u}^{k}\right)$. Using Proposition 2.4, for any $1 \leq i \leq k$, the set of vertices $\left\{\left(g_{n}\left(\mathbf{u}^{i}\right)\right)^{\uparrow m}: m \geq 1\right\}$ is not explored till the $n$th step (see Figure 2). This provides an upper bound on the size of the next step, and hence on the increment of the height of the history region. The height of the first vertex of $\left\{\left(g_{n}\left(\mathbf{u}^{i}\right)\right)^{\uparrow m}: m \geq 1\right\}$ which is open from $g_{n}\left(\mathbf{u}^{i}\right)$ is a geometric random variable irrespective of the history carried. Using these geometric random variables, we construct a coupling with a Markov chain $M_{n}$ which dominates the height random variable. Hence, the Markov chain's return time to 0 will dominate the return time of $L_{n}$ to 0 . The Markov chain is constructed so that it uses an independent sequence of random variables when $L_{n}$ has already returned to 0 but $M_{n}$ is positive. 
Remark 2.5. Note here that Proposition 2.4 remains valid even if we consider the $L_{2}$ norm and define regeneration in the same way, i.e., when the history set becomes empty. Though the geometric structure of the history region changes, we can still provide a bound on the increment of the height and construct a dominating Markov chain with the same properties as above. The geometry of the $L_{1}$ structure has been used very minimally, and wherever they have been used we may see that the results also hold when it is replaced with the $L_{2}$ norm.

To prove Proposition 2.3, we need an auxiliary lemma on Markov chains, whose proof is given in the Appendix. Let $\left\{\theta_{n}: n \geq 1\right\}$ be a sequence of i.i.d. positive integer valued random variables with $\mathbb{P}\left(\theta_{1}=1\right)>0$ and $\mathbb{P}\left(\theta_{1} \geq n\right) \leq C_{3} \exp \left(-C_{4} n\right)$ for all $n \geq 1$ where $C_{3}, C_{4}$ are positive constants. Define a sequence of random variables as follows: $M_{0}:=0$ and for $l \geq 0, M_{l+1}:=\max \left\{M_{l}, \theta_{l+1}\right\}-1$. Let $\tau^{M}:=\inf \left\{l \geq 1: M_{l}=0\right\}$ be the first return time of $M_{l}$ to 0 .

Lemma 2.6. For $n \geq 1$, we have

$$
\mathbb{P}\left(\tau^{M} \geq n\right) \leq C_{5} \exp \left(-C_{6} n\right)
$$

where $C_{5}$ and $C_{6}$ are positive constants.

Proof of Proposition 2.3; We first observe that by the Markov property (Proposition 2.2) it is enough to show the result for $l=1$. In order to study that, we define,

$$
L_{n}:= \begin{cases}\max \left\{\mathbf{w}(d): \mathbf{w} \in \Delta_{n}\right\}-r_{n} & \text { if } \Delta_{n} \neq \emptyset \\ 0 & \text { if } \Delta_{n}=\emptyset\end{cases}
$$

where $r_{n}=\min \left\{g_{n}\left(\mathbf{u}^{i}\right)(d): i=1, \ldots, k\right\}$. We set,

$$
\tau^{L}:=\inf \left\{n \geq 1: L_{n}=0\right\}
$$

and observe that $\tau_{1}=\tau^{L}$.

For any fixed $n \geq 0$, using Proposition 2.4, we have that $\left(g_{n}\left(\mathbf{u}^{i}\right)\right)^{\uparrow m}$ is unexplored for $m \geq 1$ and $1 \leq i \leq k$ (see Figure 2). We now define the collection of random variables

$$
\left\{J_{n+1}(\mathbf{w}):=\inf \left\{m \geq 1:(\mathbf{w})^{\uparrow m} \in V\right\}: \mathbf{w} \in W_{n}^{\text {move }}\right\},
$$

where $V$ is the set of all open points. This is a collection of i.i.d. geometric random variables with parameter $p$, i.e. each of the random variables takes the value $m$ with probability $p(1-p)^{m-1}$ for $m=1,2, \ldots$ Also,

$$
\left\|g_{n}(\mathbf{w})-g_{n+1}(\mathbf{w})\right\|_{1} \leq J_{n+1}(\mathbf{w}) \text { for all } \mathbf{w} \text { with } g_{n}(\mathbf{w}) \in W_{n}^{\text {move }} .
$$


Let $\left\{G_{n}^{i, 1}: 1 \leq i \leq k, n \geq 0\right\}$ be another family of i.i.d. geometric random variables with parameter $p$, independent of $\left\{U_{\mathbf{w}}: \mathbf{w} \in \mathbb{Z}^{d}\right\}$.

Now given $g_{n}\left(\mathbf{u}^{1}\right), \ldots, g_{n}\left(\mathbf{u}^{k}\right)$ and $H_{n}$, we define $\left\{M_{n}:=M_{n}\left(\mathbf{u}^{1}, \ldots, \mathbf{u}^{k}\right), X_{n}:=\right.$ $\left.X_{n}\left(\mathbf{u}^{1}, \ldots, \mathbf{u}^{k}\right): n \geq 0\right\}$ as follows:

$$
\text { set } M_{0}=0=X_{0} \text { and } M_{n+1}=\max \left\{M_{n}, J_{n+1}^{1}\right\}-1 \text { for } n \geq 0
$$

where

$$
J_{n+1}^{1}:= \begin{cases}\max \left\{J_{n+1}(\mathbf{u}): g_{n}(\mathbf{u}) \in W_{n}^{\text {move }}\right\} & \text { if } \# W_{n}^{\text {move }}=k \text { and } X_{n}=0, \\ \max \left\{G_{n+1}^{i, 1}, J_{n+1}(\mathbf{u}):\right. & \\ \left.g_{n}(\mathbf{u}) \in W_{n}^{\text {move }}, i=1, \ldots, k-k^{\prime}\right\} & \text { if } \# W_{n}^{\text {move }}=k^{\prime}<k \text { and } X_{n}=0, \\ \max \left\{G_{n+1}^{i, 1}: 1 \leq i \leq k\right\} & \text { if } X_{n}=1,\end{cases}
$$

and

$$
X_{n+1}:= \begin{cases}1 & \text { if } X_{n}=0, L_{n+1}=0 \\ X_{n} & \text { otherwise }\end{cases}
$$

From (66) it follows that $\left\{J_{n+1}^{1}: n \geq 0\right\}$ is a family of i.i.d. copies of $J$ where for any $m \geq 1$,

$$
\mathbb{P}(J \geq m)=1-\left(1-(1-p)^{m-1}\right)^{k} \leq k(1-p)^{m-1}
$$

and hence the sequence $\left\{J_{n}^{1}: n \geq 1\right\}$ satisfies the conditions of Lemma 2.6.

Further, we claim that $0 \leq L_{n} \leq M_{n}$ for all $0 \leq n \leq \tau^{L}$. Indeed, this holds for $n=0$, and assume that it holds for some $0 \leq n<\overline{\tau^{L}}$. If $\Delta_{n+1}=\emptyset$ then we have $0=L_{n+1} \leq M_{n+1}$. Otherwise if $\mathbf{w} \in \Delta_{n+1}$, then, from the definition of $\Delta_{n+1}$, either $\mathbf{w} \in \Delta_{n}$ or $\mathbf{w} \in S^{+}\left(\mathbf{u},\|\mathbf{u}-h(\mathbf{u})\|_{1}\right)$ for some $\mathbf{u} \in W_{n}^{\text {move }}$. Therefore, from (5) and (하), $\mathbf{w}(d) \leq \max \left\{\max \left\{\mathbf{u}(d): \mathbf{u} \in \Delta_{n}\right\}, \min \left\{g_{n}\left(\mathbf{u}^{i}\right)(d), 1 \leq i \leq k\right\}+\| \mathbf{u}-\right.$ $\left.h(\mathbf{u}) \|_{1}: \mathbf{u} \in W_{n}^{\text {move }}\right\} \leq \max \left\{L_{n}+r_{n}, r_{n}+J_{n+1}\right\}=\max \left\{L_{n}, J_{n+1}\right\}+r_{n}$. Also $r_{n+1}=\min \left\{g_{n+1}\left(\mathbf{u}^{i}\right)(d), 1 \leq i \leq k\right\} \geq \min \left\{g_{n}\left(\mathbf{u}^{i}\right)(d), 1 \leq i \leq k\right\}+1=r_{n}+1$. Thus $L_{n+1} \leq \max \left\{L_{n}, J_{n+1}\right\}-1 \leq \max \left\{M_{n}, J_{n+1}\right\}-1=M_{n+1}$.

Define,

$$
\tau^{M}=\tau^{M}\left(\mathbf{u}^{1}, \ldots, \mathbf{u}^{k}\right):=\inf \left\{n \geq 1: M_{n}=0\right\} .
$$

Note that the distribution of $\tau^{M}\left(\mathbf{u}^{1}, \ldots, \mathbf{u}^{k}\right)$ does not depend on $\mathbf{u}^{1}, \ldots, \mathbf{u}^{k}$. From the above observation that $0 \leq L_{n} \leq M_{n}$ for $0 \leq n \leq \tau_{1}$, we obtain that

$$
\tau_{1}=\tau^{L} \leq \tau^{M}
$$

Using Lemma 2.6, we obtain Proposition 2.3. 
The following lemma will be used to show that the inter-regeneration times as well as the width of the explored regions during a regeneration have exponentially decaying tail probabilities. Let $\left\{\theta_{i}: i \geq 1\right\}$ be i.i.d. random variables and $N$ be any random variable taking values in $\{0,1,2, \ldots\}$. We define the random sum $S$ as follows:

$$
S:= \begin{cases}0 & \text { if } N=0 \\ \sum_{i=1}^{n} \theta_{i} & \text { if } N=n\end{cases}
$$

Then, we have following lemma.

Lemma 2.7. Suppose that for some $\beta>0$ and $\alpha>0, \mathbb{E}\left(\exp \left(\beta \theta_{1}\right)\right)<\infty$ and $\mathbb{E}(\exp (\alpha N))<\infty$. Then, there exists $\gamma>0$ such that $\mathbb{E}(\exp (\gamma S))<\infty$.

We note here that no assumption of independence or the structure of dependence between $N$ and $\theta_{i}$ 's have been imposed here. The proof uses the CauchySchwartz inequality and has been relegated to the Appendix.

We now consider the width of the explored region between the $l-1$ and $l$ th regenerations. For the process starting from $\mathbf{u}^{1}, \ldots, \mathbf{u}^{k}$ with $\mathbf{u}^{1}(d)=\cdots=\mathbf{u}^{k}(d)$ define

$$
W_{l}=W_{l}\left(\mathbf{u}^{1}, \ldots, \mathbf{u}^{k}\right):=\sum_{n=\tau_{l-1}+1}^{\tau_{l}} \sum_{i=1}^{k}\left\|g_{n}\left(\mathbf{u}^{i}\right)-g_{n-1}\left(\mathbf{u}^{i}\right)\right\|_{1} .
$$

Using $\left\{G_{n}^{i, l+1}: \quad 1 \leq i \leq k, n \geq 0\right\}$, another family of i.i.d. geometric random variables with parameter $p$ and independent of both $\left\{U_{\mathbf{w}}: \mathbf{w} \in \mathbb{Z}^{d}\right\}$ and $\left\{G_{n}^{i, j}: 1 \leq i \leq k, 1 \leq j \leq l, n \geq 0\right\}$, our construction ensures that $\sigma_{l+1}\left(\mathbf{u}^{1}, \ldots, \mathbf{u}^{k}\right) \leq \tau^{M}\left(g_{\tau_{l}}\left(\mathbf{u}^{1}\right), \ldots, g_{\tau_{l}}\left(\mathbf{u}^{k}\right)\right)$ and $\tau^{M}\left(g_{\tau_{l}}\left(\mathbf{u}^{1}\right), \ldots, g_{\tau_{l}}\left(\mathbf{u}^{k}\right)\right)$ is an i.i.d. copy of $\tau^{M}\left(\mathbf{u}_{1}, \ldots, \mathbf{u}_{k}\right)$. Also, for $\tau_{l} \leq n<\tau_{l+1}$, we have

$$
\sum_{i=1}^{k}\left\|g_{n+1}\left(\mathbf{u}^{i}\right)-g_{n}\left(\mathbf{u}^{i}\right)\right\|_{1} \leq \sum_{g_{n}\left(\mathbf{u}^{i}\right) \in W_{\text {move }}^{n}} J_{n+1}\left(\mathbf{u}^{i}\right) \leq k J_{\left(n-\tau_{l}\right)+1}^{l+1}
$$

where the last sum is over distinct elements of $W_{n}^{\text {move }}$ to avoid double counting and $J_{i}^{l+1}$ is defined as in (6) using $\left\{G_{n}^{i, l+1}: 1 \leq i \leq k, n \geq 0\right\}$ instead of $\left\{G_{n}^{i, 1}\right.$ : $1 \leq i \leq k, n \geq 0\}$. Further it follows that $W_{l+1} \leq \sum_{i=1}^{\tau^{M}\left(g_{\tau_{l}}\left(\mathbf{u}_{1}\right), \ldots, g_{\tau_{l}}\left(\mathbf{u}_{k}\right)\right)} k J_{i}^{l+1}$

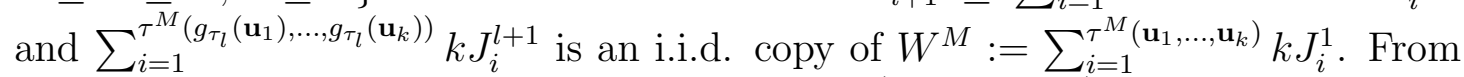
Lemma 2.7, we conclude that for some $\alpha>0, \mathbb{E}\left(\exp \left(\alpha W^{M}\right)\right)<\infty$.

The time for the $l$ th regeneration, measured by the distance travelled by process in the $d$ th co-ordinate (see Figure 44), is defined as

$$
T_{l}=T_{l}\left(\mathbf{u}^{1}, \ldots, \mathbf{u}^{k}\right):=g_{\tau_{l}}\left(\mathbf{u}^{1}\right)(d)-\mathbf{u}^{1}(d)=g_{\tau_{l}}\left(\mathbf{u}^{i}\right)(d)-\mathbf{u}^{i}(d) \text { for } 1 \leq i \leq k .
$$

Clearly $T_{l}-T_{l-1} \leq W_{l}$ and from the fact that, for some $\alpha>0, \mathbb{E}\left(\exp \left(\alpha W^{M}\right)\right)<\infty$, we conclude the following Proposition. 
Proposition 2.8. For any $l \geq 1$ and $\mathbf{u}^{1}, \ldots, \mathbf{u}^{k}$ with $\mathbf{u}^{1}(d)=\cdots=\mathbf{u}^{k}(d)$, we have

$$
\mathbb{P}\left(T_{l}-T_{l-1} \geq n\right) \leq \mathbb{P}\left(W_{l} \geq n\right) \leq \mathbb{P}\left(W^{M} \geq n\right) \leq C_{7}^{(k)} \exp \left(-C_{8}^{(k)} n\right)
$$

for all $n \geq 1$, where $C_{7}^{(k)}$ and $C_{8}^{(k)}$ are positive constants, depending on $k$ but not $l$ or $\mathbf{u}^{1}, \ldots, \mathbf{u}^{k}$.

Now we specialize to the case $k=1$, i.e., the process starting from just one vertex $\mathbf{u}$ and consider the inter-regeneration step process $\sigma_{l}(\mathbf{u})$, the width process $W_{l}(\mathbf{u})$ and the inter-regeneration time process $T_{l}(\mathbf{u})-T_{l-1}(\mathbf{u})$, defined in (1), (9) and (10) respectively. Using the Markov property of $\left\{\mathcal{Z}_{l}^{(1)}: l \geq 0\right\}$ and the translation invariance of the model, we have that $\left\{\left(\sigma_{l}(\mathbf{u}), W_{l}(\mathbf{u}),\left(T_{l}(\mathbf{u})-T_{l-1}(\mathbf{u})\right)\right)\right.$ : $l \geq 1\}$ is an i.i.d. family of random vectors taking values in $\{1,2,3, \ldots\}^{3}$. Using the translation invariance of our model, the distribution of these random variables does not depend on the choice of the starting vertex $\mathbf{u}$. Furthermore, each of the marginals of this random vector has exponentially decaying tail probability. Let

$$
\overline{\mathbf{w}}:=(\mathbf{w}(1), \ldots, \mathbf{w}(d-1)) \text { for } \mathbf{w}=(\mathbf{w}(1), \ldots, \mathbf{w}(d))
$$

denote the first $d-1$ co-ordinates of $\mathbf{w} \in \mathbb{Z}^{d}$. We observe the following:

Remark 2.9. Define, for any $l \geq 1$,

$$
Y_{l}^{(\mathbf{u})}:=\bar{g}_{\tau_{l}(\mathbf{u})}(\mathbf{u})-\bar{g}_{\tau_{l-1}(\mathbf{u})}(\mathbf{u}) .
$$

We have

(a) $\left\{Y_{l}^{(\mathbf{u})}: l \geq 1\right\}$ is a sequence of i.i.d. $\mathbb{Z}^{d-1}$-valued random vectors, whose distribution does not depend on $\mathbf{u}$. For $\mathbf{u}=\mathbf{0}$, we denote $Y_{l}^{(\mathbf{0})}$ by $Y_{l}$.

(b) Denoting the $L_{1}$ norm in $(d-1)$ dimensions by $\|\cdot\|_{1, d-1}$, we also observe that $\left\|Y_{1}\right\|_{1, d-1} \leq W_{1}(\mathbf{0})$, so that we also have

$$
\mathbb{P}\left(\left\|Y_{1}\right\|_{1, d-1} \geq n\right) \leq C_{7}^{(1)} \exp \left(-C_{8}^{(1)} n\right)
$$

for all $n \geq 1$, where $C_{7}^{(1)}$ and $C_{8}^{(1)}$ are as in (11).

(c) By reflection symmetry of the model, about any of the first $(d-1)$ coordinates, we have that each co-ordinate of $Y_{1}$ is marginally symmetric. Further, the rotational symmetry of the model in the first $(d-1)$ co-ordinates implies that the marginal distributions are same for $i=1,2, \ldots, d-1$. In other words, $\mathbb{P}\left(Y_{1}(1)=+m\right)=\mathbb{P}\left(Y_{1}(i)=+m\right)=\mathbb{P}\left(Y_{1}(i)=-m\right)$ for all $m \geq 1$ and $1 \leq i \leq d-1$ where $Y_{1}(i)$ denotes the $i$ th co-ordinate of $Y_{1}$. 
(d) For any $i, j \in\{1,2, \ldots, d-1\}, i \neq j$, let $s=\mathbb{E}\left(Y_{1}(i) Y_{1}(j)\right)$. By reflection symmetry along the $i$ th co-ordinate, with other co-ordinates being fixed, we observe that the joint distribution of $\left(Y_{1}(i), Y_{1}(j)\right)$ remains unchanged. This implies that $s=\mathbb{E}\left(\left(-Y_{1}(i)\right) Y_{1}(j)\right)$ and hence, $\mathbb{E}\left(Y_{1}(i) Y_{1}(j)\right)=0$. The same argument holds to prove $\mathbb{E}\left[\left(Y_{1}(i)\right)^{m_{1}}\left(Y_{1}(j)\right)^{m_{2}}\right]=0$ for $m_{1}, m_{2} \geq 1$ with at least one of them being odd.

\section{$3 \quad$ Martingale and independent processes}

In this section, we study the joint evolution of paths starting from two vertices $\mathbf{u}$ and $\mathbf{v}$ with $\mathbf{u}(d)=\mathbf{v}(d)$. The process $\left\{\left(g_{\tau_{l}}(\mathbf{u}), g_{\tau_{l}}(\mathbf{v})\right): l \geq 0\right\}$ is a Markov chain on $\left(\mathbb{Z}^{d}\right)^{2}$ by Corollary 2.3.1. Further, our model is translation invariant. Hence, the process $\left\{g_{\tau_{l}}(\mathbf{u})-g_{\tau_{l}}(\mathbf{v}): l \geq 0\right\}$ is also a Markov chain on $\mathbb{Z}^{d}$. However, as observed earlier, (see Figure 4),$g_{\tau_{l}}(\mathbf{u})(d)=g_{\tau_{l}}(\mathbf{v})(d)$ for every $l \geq 1$. Thus, using notation as in (12),

$$
\left\{Z_{l}=Z_{l}(\mathbf{u}, \mathbf{v}):=\bar{g}_{\tau_{l}}(\mathbf{u})-\bar{g}_{\tau_{l}}(\mathbf{v}): l \geq 0\right\}
$$

is a $\mathbb{Z}^{d-1}$ valued Markov chain. Further, we observe that $\overline{\mathbf{0}}=(\underbrace{0,0, \ldots, 0}_{d-1})$ is the only absorbing state of this Markov chain.

In Subsection 3.1. we prove that the process $Z_{l}$ is a martingale for $d=2$. Later, in Subsection 3.2 and Subsection 3.3. we study, for any $d \geq 2$, how $Z_{l}$ can be approximated by a process having i.i.d. increments when the starting point of paths are sufficiently far apart.

\subsection{Martingale}

In this subsection we restrict ourselves to $d=2$ and fix two vertices $\mathbf{u}, \mathbf{v} \in \mathbb{Z}^{2}$ with $\mathbf{u}(2)=\mathbf{v}(2)$. We first observe that, for $l \geq 0$, the regeneration time $T_{l}=T_{l}(\mathbf{u}, \mathbf{v})$ is a stopping time with respect to the filtration $\left\{\mathcal{F}_{t}: t \geq 0\right\}$ where $\mathcal{F}_{t}:=\sigma\left\{U_{\mathbf{w}}\right.$ : $\mathbf{w}(2) \leq \mathbf{u}(2)+t\}$. By our construction, $g_{\tau_{l}}(\mathbf{u})$ is $\mathcal{F}_{T_{l}}$ measurable. Therefore, $\bar{g}_{\tau_{l}}(\mathbf{u})$, given by the projection from $\mathbb{Z}^{2} \rightarrow \mathbb{Z}$, is also $\mathcal{F}_{T_{l}}$ measurable.

Proposition 3.1. For $d=2$ and $\mathbf{u}, \mathbf{v} \in \mathbb{Z}^{2}$ with $\mathbf{u}(2)=\mathbf{v}(2)$, the process $\left\{\bar{g}_{\tau_{l}}(\mathbf{u})\right.$ : $l \geq 0\}$ is a martingale with respect to the filtration $\left\{\mathcal{F}_{T_{l}}: l \geq 0\right\}$.

The above proposition also holds for $\bar{g}_{\tau_{l}}(\mathbf{v})$, so we obtain

Corollary 3.1.1. For $d=2$ and any $\mathbf{u}, \mathbf{v} \in \mathbb{Z}^{2}$ with $\mathbf{u}(2)=\mathbf{v}(2)$, the process $\left\{Z_{l}=Z_{l}(\mathbf{u}, \mathbf{v}): l \geq 0\right\}$ is a martingale with respect to the filtration $\left\{\mathcal{F}_{T_{l}}: l \geq 0\right\}$. 
Proof of Proposition 3.1. Consider the process $\left(g_{n}(\mathbf{u}), g_{n}(\mathbf{v}), \Delta_{n}(\mathbf{u}, \mathbf{v}), \Psi_{n}\right)$ starting from $\mathbf{u}, \mathbf{v}$ with $\mathbf{u}(2)=\mathbf{v}(2)$, and the process $\left(g_{n}(\mathbf{u}), \Delta_{n}(\mathbf{u}), \Psi_{n}\right)$ starting from $\mathbf{u}$ with the same set of uniform random variables $\left\{U_{\mathbf{w}}: \mathbf{w} \in \mathbb{Z}^{2}\right\}$. Observe that every joint regeneration of the paths from a pair of vertices $\mathbf{u}, \mathbf{v}$ is also a regeneration of the single path from $\mathbf{u}$, i.e., for every $l \geq 0$, we have

$$
T_{l}(\mathbf{u}, \mathbf{v})=T_{N_{l}}(\mathbf{u})
$$

for some sequence $N_{l}=N_{l}(\mathbf{u}, \mathbf{v})$ (see Figure 4 where $N_{1}=2$ for $\mathbf{u}^{1}$ and $N_{1}=3$ for $\left.\mathbf{u}^{2}\right)$. Therefore, we have,

$$
\bar{g}_{\tau_{l}(\mathbf{u}, \mathbf{v})}(\mathbf{u})=\bar{g}_{\tau_{N_{l}}(\mathbf{u})}(\mathbf{u})=\sum_{i=1}^{N_{l}} Y_{i}^{(\mathbf{u})}
$$

where $Y_{i}^{(\mathbf{u})}:=\bar{g}_{\tau_{i}(\mathbf{u})}(\mathbf{u})-\bar{g}_{\tau_{i-1}(\mathbf{u})}(\mathbf{u})$ is as in Remark 2.9. Since $N_{l} \leq T_{l}(\mathbf{u}, \mathbf{v})$, and each of $T_{i}(\mathbf{u}, \mathbf{v})-T_{i-1}(\mathbf{u}, \mathbf{v})$ and $Y_{i}^{(\mathbf{u})}$ has an exponentially decaying tail probability (see Proposition 2.8 and equation (14)), for every $l \geq 0$, we have that $\mathbb{E}\left(\left|\bar{g}_{\tau_{l}(\mathbf{u}, \mathbf{v})}(\mathbf{u})\right|\right)<\infty$.

Further we need to show that

$$
\mathbb{E}\left[\bar{g}_{\tau_{l+1}(\mathbf{u}, \mathbf{v})}(\mathbf{u})-\bar{g}_{\tau_{l}(\mathbf{u}, \mathbf{v})}(\mathbf{u}) \mid \mathcal{F}_{T_{l}(\mathbf{u}, \mathbf{v})}\right]=\mathbb{E}\left[\sum_{i=N_{l}+1}^{N_{l+1}} Y_{i}^{(\mathbf{u})} \mid \mathcal{F}_{T_{N_{l}}(\mathbf{u})}\right]=0 \text { a.s. }
$$

Denoting $\mathcal{G}_{i}:=\mathcal{F}_{T_{i}(\mathbf{u})}$, we have that $Y_{i+1}^{(\mathbf{u})}$ is independent of $\mathcal{G}_{i}$. We also observe that $N_{l}$ is $\left\{\mathcal{G}_{i}: i \geq 0\right\}$ adapted for each $l \geq 0$, i.e., $\left\{N_{l} \leq m\right\} \in \mathcal{G}_{m}$. Therefore, 
using 1 to denote the indicator function, for any $A \in \mathcal{F}_{T_{N_{l}}(\mathbf{u})}=\mathcal{G}_{N_{l}}$, we have

$$
\begin{aligned}
\mathbb{E} & {\left[\mathbf{1}(A) \sum_{i=N_{l}+1}^{N_{l+1}} Y_{i}^{(\mathbf{u})}\right] } \\
= & \mathbb{E}\left[\mathbf{1}(A) \sum_{n_{l}=1}^{\infty} \sum_{m=1}^{\infty} \mathbf{1}\left(N_{l}=n_{l}\right) \mathbf{1}\left(N_{l+1}=n_{l}+m\right) \sum_{i=1}^{m} Y_{n_{l}+i}^{(\mathbf{u})}\right] \\
= & \mathbb{E}\left[\mathbf{1}(A) \sum_{n_{l}=1}^{\infty} \sum_{m=1}^{\infty} \mathbf{1}\left(N_{l}=n_{l}\right) \mathbf{1}\left(N_{l+1} \geq n_{l}+m\right) Y_{n_{l}+m}^{(\mathbf{u})}\right] \\
= & \sum_{n_{l}=1}^{\infty} \sum_{m=1}^{\infty} \mathbb{E}\left[\mathbf{1}(A) \mathbf{1}\left(N_{l}=n_{l}\right)\left[1-\mathbf{1}\left(N_{l+1} \leq n_{l}+m-1\right)\right] Y_{n_{l}+m}^{(\mathbf{u})}\right] \\
= & \sum_{n_{l}=1}^{\infty} \sum_{m=1}^{\infty} \mathbb{E}\left[\mathbb{E}\left[\mathbf{1}(A) \mathbf{1}\left(N_{l}=n_{l}\right)\left[1-\mathbf{1}\left(N_{l+1} \leq n_{l}+m-1\right)\right] Y_{n_{l}+m}^{(\mathbf{u})} \mid \mathcal{G}_{n_{l}+m-1}\right]\right] \\
= & \sum_{n_{l}=1}^{\infty} \sum_{m=1}^{\infty} \mathbb{E}\left[\mathbf{1}(A) \mathbf{1}\left(N_{l}=n_{l}\right)\left[1-\mathbf{1}\left(N_{l+1} \leq n_{l}+m-1\right)\right] \mathbb{E}\left[Y_{n_{l}+m}^{(\mathbf{u})} \mid \mathcal{G}_{n_{l}+m-1}\right]\right] \\
= & \sum_{n_{l}=1}^{\infty} \sum_{m=1}^{\infty} \mathbb{E}\left[\mathbf{1}(A) \mathbf{1}\left(N_{l}=n_{l}\right)\left[1-\mathbf{1}\left(N_{l+1} \leq n_{l}+m-1\right)\right] \mathbb{E}\left[Y_{n_{l}+m}^{(\mathbf{u})}\right]\right] \\
= & 0
\end{aligned}
$$

In the above, we have used Fubini's theorem to interchange the expectation and summation. Observe that $\mathbf{1}(A) \sum_{i=N_{l}+1}^{N_{l+1}}\left|Y_{i}^{(\mathbf{u})}\right| \leq W_{l+1}(\mathbf{u}, \mathbf{v})$. Hence, using Proposition 2.8, $\mathbb{E}\left(\mathbf{1}(A) \sum_{i=N_{l}+1}^{N_{l+1}}\left|Y_{i}^{(\mathbf{u})}\right|\right)<\infty$. In the above we have also used property (c) of Remark 2.9 and the fact that, since $A \in \mathcal{G}_{N_{l}}, A \cap\left\{N_{l}=n_{l}\right\} \in \mathcal{G}_{n_{l}} \subseteq \mathcal{G}_{n_{l}+m-1}$ for all $m \geq 1$. Also, $\left\{N_{l+1} \leq n_{l}+m-1\right\} \in \mathcal{G}_{n_{l}+m-1}$ and $Y_{n_{l}+m}^{(\mathbf{u})}$ is independent of $\mathcal{G}_{n_{l}+m-1}$.

\subsection{Independent processes}

In this subsection, we describe simultaneous regenerations of two independent paths. This will be used to approximate the paths at simultaneous regenerations of joint paths when the starting points are far apart. We start with a result (Lemma 3.2) about renewal processes, which is proved in the Appendix.

Let $\left\{\xi_{n}^{(1)}: n \geq 1\right\}$ and $\left\{\xi_{n}^{(2)}: n \geq 1\right\}$ be two independent collections of i.i.d. inter-arrival times (positive integer valued random variables) with $\mathbb{P}\left(\xi_{1}^{(1)}=j\right)=$ $f_{j}^{(1)}$ and $\mathbb{P}\left(\xi_{1}^{(2)}=j\right)=f_{j}^{(2)}$. We assume that, for any $m \geq 1, \max \left\{\mathbb{P}\left(\xi_{1}^{(1)} \geq\right.\right.$ $\left.m), \mathbb{P}\left(\xi_{1}^{(2)} \geq m\right)\right\} \leq C_{9} \exp \left(-C_{10} m\right)$ where $C_{9}$ and $C_{10}$ are positive constants 
and $f_{1}^{(1)} f_{1}^{(2)}>0$. Let $S_{0}^{(1)}:=0=: S_{0}^{(2)}$ and, for $n \geq 1, S_{n}^{(1)}:=\sum_{j=1}^{n} \xi_{j}^{(1)}$ and $S_{n}^{(2)}:=\sum_{j=1}^{n} \xi_{j}^{(2)}$. For any $n \geq 1$ and $i=1,2$, define the residual life of the $i$ th component at time $n$ by

$$
R_{n}^{(i)}:=\inf \left\{S_{k}^{(i)}: S_{k}^{(i)} \geq n\right\}-n .
$$

We consider the joint residual process $\left(R_{n}^{(i)}: i=1,2\right)$ and define

$$
\tau^{R}:=\inf \left\{n \geq 1: R_{n}^{(1)}=R_{n}^{(2)}=0\right\} .
$$

Lemma 3.2. For any $n \geq 1$, we have

$$
\mathbb{P}\left(\tau^{R} \geq n\right) \leq C_{11} \exp \left(-C_{12} n\right)
$$

where $C_{11}$ and $C_{12}$ are positive constants, depending on the distribution of $\xi_{n}^{(i)}$ 's only.

Now we fix two vertices $\mathbf{u}$ and $\mathbf{v}$ with $\mathbf{u}(d)=\mathbf{v}(d)$ and consider two independent constructions of the marginal paths, one starting from $\mathbf{u}$ and the other from $\mathbf{v}$. More precisely, we start with two independent collections of uniform i.i.d. random variables, $\left\{U_{\mathbf{w}}^{\mathbf{u}}: \mathbf{w} \in \mathbb{Z}^{d}\right\}$ and $\left\{U_{\mathbf{w}}^{\mathbf{v}}: \mathbf{w} \in \mathbb{Z}^{d}\right\}$. Now, as in Section 2, we start two paths, one from $\mathbf{u}$, constructed using the collection $\left\{U_{\mathbf{w}}^{\mathbf{u}}: \mathbf{w} \in \mathbb{Z}^{d}\right\}$, and the other from $\mathbf{v}$, constructed using the collection $\left\{U_{\mathbf{w}}^{\mathbf{v}}: \mathbf{w} \in \mathbb{Z}^{d}\right\}$. We denote these respective paths by $\left\{g_{n}^{(\text {Ind) }}(\mathbf{u}): n \geq 0\right\}$ and $\left\{g_{n}^{(\text {Ind) }}(\mathbf{v}): n \geq 0\right\}$. The above processes being independent, we have two independent collections of regeneration times, $\left\{T_{l}^{(\text {Ind) }}(\mathbf{u}): l \geq 0\right\}$ and $\left\{T_{l}^{(\text {Ind) }}(\mathbf{v}): l \geq 0\right\}$ (see equation (10) for definition). As mentioned in Remark 2.9, for a single starting point, the distribution of the collection $\left\{T_{l}^{(\text {Ind) }}(\mathbf{u}): l \geq 0\right\}$ or $\left\{T_{l}^{(\text {Ind })}(\mathbf{v}): l \geq 0\right\}$ does not depend on the starting point, and is an independent copy of $\left\{T_{l}(\mathbf{0}): l \geq 0\right\}$.

Take $R_{n}^{(1)}=\inf \left\{T_{l}^{(\text {Ind) }}(\mathbf{u}): T_{l}^{(\text {Ind) }}(\mathbf{u}) \geq n\right\}-n$ and $R_{n}^{(2)}=\inf \left\{T_{l}^{(\text {Ind })}(\mathbf{v}):\right.$ $\left.T_{l}^{(\text {Ind) }}(\mathbf{v}) \geq n\right\}-n$. Note here that $\left\{T_{l+1}^{(\text {Ind })}(\mathbf{u})-T_{l}^{(\text {Ind) }}(\mathbf{u}): l \geq 0\right\}$ and $\left\{T_{l+1}^{(\text {Ind })}(\mathbf{v})-\right.$ $\left.T_{l}^{(\text {Ind) }}(\mathbf{v}): l \geq 0\right\}$ are two independent collections of i.i.d. random variables, which play the respective roles of $\left\{\xi_{l}^{(1)}: l \geq 1\right\}$ and $\left\{\xi_{l}^{(2)}: l \geq 1\right\}$ of Lemma 3.2. Set, $T_{0}^{(\text {Ind })}(\mathbf{u}, \mathbf{v})=0$ and, for $l \geq 0$,

$$
T_{l+1}^{(\mathrm{Ind})}(\mathbf{u}, \mathbf{v}):=\inf \left\{n>T_{l}^{(\mathrm{Ind})}(\mathbf{u}, \mathbf{v}): R_{n}^{(1)}=R_{n}^{(2)}=0\right\} .
$$

We call $T_{l}^{(\mathrm{Ind})}(\mathbf{u}, \mathbf{v})$, the time for the $l$ th simultaneous regeneration time of two independent paths.

Applying Lemma 3.2 and observing that each $T_{l}^{(\mathrm{Ind})}(\mathbf{u}, \mathbf{v})$ represents the occurrence of a renewal event, we obtain the following proposition. 
Proposition 3.3. $\left\{T_{l+1}^{(\mathrm{Ind})}(\mathbf{u}, \mathbf{v})-T_{l}^{(\mathrm{Ind})}(\mathbf{u}, \mathbf{v}): l \geq 0\right\}$ is an i.i.d. sequence of random variables taking values in $\{1,2,3, \ldots\}$ and, for all $n \geq 1$,

$$
\mathbb{P}\left(T_{1}^{(\mathrm{Ind})}(\mathbf{u}, \mathbf{v}) \geq n\right) \leq C_{13} \exp \left(-C_{14} n\right)
$$

where $C_{13}$ and $C_{14}$ are positive constants.

Any simultaneous regeneration time of two independent paths $T_{l}^{(\mathrm{Ind})}(\mathbf{u}, \mathbf{v})$ is also a regeneration time for each of the marginal processes. Therefore, as in (16), for every $l \geq 0$, we have

$$
T_{l}^{(\mathrm{Ind})}(\mathbf{u}, \mathbf{v})=T_{N_{l}^{\mathbf{u}}}^{(\mathrm{Ind})}(\mathbf{u})=T_{N_{l}^{\mathbf{v}}}^{(\mathrm{Ind})}(\mathbf{v})
$$

for some sequences $N_{l}^{\mathbf{u}}\left(=N_{l}^{\mathbf{u}}(\mathbf{u}, \mathbf{v})\right)$ and $N_{l}^{\mathbf{v}}\left(=N_{l}^{\mathbf{v}}(\mathbf{u}, \mathbf{v})\right)$ with $N_{0}^{\mathbf{u}}=N_{0}^{\mathbf{v}}=0$.

As in (9) consider the width of the explored region between the $l-1$ and $l$ th regenerations of both the independent processes,

$$
W_{l}^{(\text {Ind })}(\mathbf{u}, \mathbf{v}):=\sum_{t=N_{l-1}^{\mathbf{u}}+1}^{N_{l}^{\mathbf{u}}} W_{t}^{(\text {Ind })}(\mathbf{u})+\sum_{t=N_{l-1}^{\mathbf{v}}+1}^{N_{l}^{\mathbf{v}}} W_{t}^{(\text {Ind })}(\mathbf{v})
$$

where $\left\{W_{l}^{(\mathrm{Ind})}(\mathbf{u}): l \geq 1\right\}$ and $\left\{W_{l}^{(\mathrm{Ind})}(\mathbf{v}): l \geq 1\right\}$ are the explored width processes associated with the processes $\left\{g_{l}^{(\text {Ind) }}(\mathbf{u}): l \geq 0\right\}$ and $\left\{g_{l}^{(\text {Ind) }}(\mathbf{v}): l \geq 0\right\}$ respectively. Observe that $\left\{\left(N_{l+1}^{\mathbf{u}}-N_{l}^{\mathbf{u}}, N_{l+1}^{\mathbf{v}}-N_{l}^{\mathbf{v}}\right): l \geq 0\right\}$ is a sequence of i.i.d. random variables, and hence, $\left\{W_{l}^{(\text {Ind) }}(\mathbf{u}, \mathbf{v}): l \geq 1\right\}$ is also a sequence of i.i.d. random variables.

Since $\max \left\{N_{1}^{\mathbf{u}}, N_{1}^{\mathbf{v}}\right\} \leq T_{1}^{(\mathrm{Ind})}(\mathbf{u}, \mathbf{v})$ and $T_{1}^{(\mathrm{Ind})}(\mathbf{u}, \mathbf{v})-T_{0}^{(\mathrm{Ind})}(\mathbf{u}, \mathbf{v})=T_{1}^{(\mathrm{Ind})}(\mathbf{u}, \mathbf{v})$ satisfies (20), using Lemma 2.7, we conclude, for any $l \geq 1$

$$
\mathbb{P}\left(W_{l}^{(\text {Ind })}(\mathbf{u}, \mathbf{v}) \geq n\right) \leq C_{15} \exp \left(-C_{16} n\right)
$$

where $C_{15}$ and $C_{16}$ are positive constants.

From (13), we can write, for any $l \geq 1$,

$$
\bar{g}_{\tau_{N_{l}}^{\mathbf{u}}}^{(\text {Ind) }}(\mathbf{u})=\overline{\mathbf{u}}+\sum_{t=1}^{N_{l}^{\mathbf{u}}} Y_{t}^{(\mathbf{u})} \quad \text { and } \quad \bar{g}_{\tau_{N_{l}}^{\mathbf{v}}}^{(\text {Ind })}(\mathbf{v})=\overline{\mathbf{v}}+\sum_{t=1}^{N_{l}^{\mathbf{v}}} Y_{t}^{(\mathbf{v})} .
$$

At each simultaneous regeneration time $T_{l}^{(\mathrm{Ind})}(\mathbf{u}, \mathbf{v})$, the $d$ th co-ordinates of both the paths coincide and equal $T_{l}^{(\mathrm{Ind})}(\mathbf{u}, \mathbf{v})$. We consider the first $d-1$ co-ordinates of these paths and define

$$
\begin{aligned}
& \psi_{l}^{\mathbf{u}}:=\bar{g}_{\tau_{N_{l}}^{\mathbf{u}}}^{\text {(Ind) }}(\mathbf{u})-\bar{g}_{\tau_{N_{l-1}}^{\mathbf{u}}}^{\text {(Ind) }}(\mathbf{u})=\sum_{t=N_{l-1}^{\mathrm{u}}+1}^{N_{l}^{\mathbf{u}}} Y_{t}^{(\mathbf{u})} \\
& \psi_{l}^{\mathbf{v}}:=\bar{g}_{\tau_{N_{l}}}^{\text {(Ind) }}(\mathbf{v})-\bar{g}_{\tau_{N_{l-1}}^{\mathrm{v}}}^{\text {(Ind) }}(\mathbf{v})=\sum_{t=N_{l-1}^{\mathbf{v}}+1}^{N_{l}^{\mathbf{v}}} Y_{t}^{(\mathbf{v})} .
\end{aligned}
$$


The process $\left(\psi_{l}^{\mathbf{u}}, \psi_{l}^{\mathbf{v}}\right)$ represents the increment between the $(l-1)$ th and $l$ th simultaneous regeneration times in the first $(d-1)$ co-ordinates of the independent paths starting from $\mathbf{u}$ and $\mathbf{v}$ respectively. We list the properties of $\left(\psi_{l}^{\mathbf{u}}, \psi_{l}^{\mathbf{v}}\right)$ in the following proposition.

Proposition 3.4. The family $\left\{\left(\psi_{l}^{\mathbf{u}}, \psi_{l}^{\mathbf{v}}\right): l \geq 1\right\}$ is an i.i.d. collection of random variables taking values in $\mathbb{Z}^{2(d-1)}$ satisfying the following properties.

(a) For any $n \geq 1$,

$$
\mathbb{P}\left(\left\|\psi_{1}^{\mathbf{u}}\right\|_{1, d-1}+\left\|\psi_{1}^{\mathbf{v}}\right\|_{1, d-1} \geq n\right) \leq C_{15} \exp \left(-C_{16} n\right)
$$

where $C_{15}$ and $C_{16}$ are as in (22).

(b) The marginal distribution of each co-ordinate of $\psi_{1}^{\mathbf{u}}$ as well as $\psi_{1}^{\mathbf{v}}$ is symmetric. Further, they are all same. More precisely, with $\psi_{l}^{\mathbf{u}}(j)$ and $\psi_{l}^{\mathbf{v}}(j)$ being the $j$ th co-ordinate of $\psi_{l}^{\mathbf{u}}$ and $\psi_{l}^{\mathbf{v}}$ respectively, $\mathbb{P}\left(\psi_{1}^{\mathbf{u}}(j)=r\right)=$ $\mathbb{P}\left(\psi_{1}^{\mathbf{u}}(j)=-r\right)=\mathbb{P}\left(\psi_{1}^{\mathbf{v}}(j)=r\right)=\mathbb{P}\left(\psi_{1}^{\mathbf{v}}(j)=-r\right)=\mathbb{P}\left(\psi_{1}^{\mathbf{u}}(1)=r\right)$ for all $j=1,2, \ldots, d-1$ and $r \geq 1$.

(c) $\mathbb{E}\left[\left(\psi_{1}^{\mathbf{u}}(i)\right)^{m_{1}}\left(\psi_{1}^{\mathbf{v}}(j)\right)^{m_{2}}\right]$ is independent of $i, j$ and depends only on $m_{1}, m_{2}$ and $\mathbb{E}\left[\left(\psi_{1}^{\mathbf{u}}(i)\right)^{m_{1}}\left(\psi_{1}^{\mathbf{v}}(j)\right)^{m_{2}}\right]=0$ if at least one of $m_{1}, m_{2}$ is odd.

Proof: Noting that $T_{l}^{(\mathrm{Ind})}(\mathbf{u}, \mathbf{v})$ represents the occurrence of a renewal event and using the fact that the families of i.i.d. random variables $\left\{Y_{t}^{(\mathbf{u})}: t \geq 1\right\}$ and $\left\{Y_{t}^{(\mathbf{v})}: t \geq 1\right\}$ are independent, we conclude that $\left\{\left(\psi_{l}^{\mathbf{u}}, \psi_{l}^{\mathbf{v}}\right): l \geq 1\right\}$ is a family of i.i.d. random variables. Further, $\left\|\psi_{1}^{(\mathbf{u})}\right\|_{1, d-1}+\left\|\psi_{1}^{(\mathbf{v})}\right\|_{1, d-1} \leq W_{1}^{(\text {Ind })}(\mathbf{u}, \mathbf{v})$. Using (22) , we conclude part (a).

Using the fact that $Y_{t}^{(\mathbf{u})}$ is symmetric in each co-ordinate, we conclude $\mathbb{P}\left(\psi_{1}^{\mathbf{u}}(j)=\right.$ $r)=\mathbb{P}\left(\psi_{1}^{\mathbf{u}}(j)=-r\right)=\mathbb{P}\left(\psi_{1}^{\mathbf{v}}(j)=r\right)=\mathbb{P}\left(\psi_{1}^{\mathbf{v}}(j)=-r\right)$ for any $r \geq 1$ for all $j=1, \ldots, d-1$. Further, $Y_{t}^{(\mathbf{u})}$ is rotation invariant along any of the first $d-1$ co-ordinates and hence $\mathbb{P}\left(\psi_{1}^{\mathbf{u}}(j)=r\right)=\mathbb{P}\left(\psi_{1}^{\mathbf{u}}(1)=r\right)$ for $j=2,3, \ldots, d-1$. This proves (b).

To study the joint distribution of $\left(\psi_{1}^{\mathbf{u}}(i), \psi_{1}^{\mathbf{v}}(j)\right)$, we observe that we may apply the rotation operator independently for both families $\left\{U_{\mathbf{w}}^{\mathbf{u}}\right\}$ and $\left\{U_{\mathbf{w}}^{\mathbf{v}}\right\}$ of uniform random variables, so that the $i$ th co-ordinate after rotation becomes the first coordinate for $\left\{U_{\mathbf{w}}^{\mathbf{u}}\right\}$ and $j$ th co-ordinate after rotation becomes the first co-ordinate for $\left\{U_{\mathbf{w}}^{\mathbf{v}}\right\}$. The distribution of $\left(Y_{t}^{(\mathbf{u})}(i), Y_{t}^{(\mathbf{v})}(j)\right)$ after rotation remains unchanged, which implies that the joint distribution of $\left(\psi_{1}^{\mathbf{u}}(i), \psi_{1}^{\mathbf{v}}(j)\right)$ is independent of $i, j$. Thus, $\mathbb{E}\left[\left(\psi_{1}^{\mathbf{u}}(i)\right)^{m_{1}}\left(\psi_{1}^{\mathbf{v}}(j)\right)^{m_{2}}\right]$ is independent of choice of $i, j$. If we fix the realizations for one family of uniform random variables and reflect the realizations of the 
other family along some co-ordinate, the distribution of $\left(Y_{t}^{(\mathbf{u})}(i), Y_{t}^{(\mathbf{v})}(j)\right)$ remains unaltered. Therefore, we have $\mathbb{P}\left(\left(\psi_{1}^{\mathbf{u}}(i), \psi_{1}^{\mathbf{v}}(j)\right)=(l, k)\right)=\mathbb{P}\left(\left(\psi_{1}^{\mathbf{u}}(i), \psi_{1}^{\mathbf{v}}(j)\right)=\right.$ $(l,-k))$ for any $l, k \in \mathbb{Z}$. This proves $(\mathrm{c})$.

Remark 3.5. Let $\xi_{l}:=\psi_{l}^{\mathbf{v}}-\psi_{l}^{\mathbf{u}}$ for $l \geq 1$ and set $S_{0}:=\overline{\mathbf{v}}-\overline{\mathbf{u}}$ and for $n \geq$ $1, S_{n}:=S_{0}+\sum_{l=1}^{n} \xi_{l}$. From the above Proposition, we conclude that the process $\left\{S_{n}: n \geq 0\right\}$ is an isotropic, symmetric random walk starting from $\overline{\mathbf{v}}-\overline{\mathbf{u}}$ on $\mathbb{Z}^{d-1}$ with i.i.d. steps, each step having exponentially decaying tail probability. Clearly, $\mathbb{P}\left(\xi_{l}=\mathbf{0}\right) \geq p^{2}$ so that the random walk is also aperiodic. This will be used in the next section.

\subsection{Coupling of joint process and independent process}

In this subsection, we describe a coupling of two independent paths with the joint paths starting from $\mathbf{u}, \mathbf{v}$ with $\mathbf{u}(d)=\mathbf{v}(d)$. Without loss of generality, we may assume $\mathbf{u}(d)=0$. Set $d_{\min }:=\|\mathbf{u}-\mathbf{v}\|_{1}$. As in the last subsection, we start with two independent collections of i.i.d. uniform random variables $\left\{U_{\mathbf{w}}^{\mathbf{u}}: \mathbf{w} \in\right.$ $\left.\mathbb{Z}^{d}, \mathbf{w}(d)>0\right\}$ and $\left\{U_{\mathbf{w}}^{\mathbf{v}}: \mathbf{w} \in \mathbb{Z}^{d}, \mathbf{w}(d)>0\right\}$, and construct two independent paths, $\left\{g_{n}^{\text {(Ind) }}(\mathbf{u}): n \geq 0\right\}$ and $\left\{g_{n}^{(\text {Ind) }}(\mathbf{v}): n \geq 0\right\}$, starting from $\mathbf{u}$ and $\mathbf{v}$ respectively.

Fix $r<d_{\min } / 2$ and another independent collection of uniform random variables $\left\{U_{\mathbf{w}}: \mathbf{w} \in \mathbb{Z}^{d}, \mathbf{w}(d)>0\right\}$. We define a new collection of uniform random variables $\left\{\tilde{U}_{\mathbf{w}}: \mathbf{w} \in \mathbb{Z}^{d}, \mathbf{w}(d)>0\right\}$ by

$$
\tilde{U}_{\mathbf{w}}:= \begin{cases}U_{\mathbf{w}}^{\mathbf{u}} & \text { if }\|\overline{\mathbf{w}}-\overline{\mathbf{u}}\|_{1, d-1}<r \\ U_{\mathbf{w}}^{\mathbf{v}} & \text { if }\|\overline{\mathbf{w}}-\overline{\mathbf{v}}\|_{1, d-1}<r \\ U_{\mathbf{w}} & \text { otherwise. }\end{cases}
$$

Using the collection $\left\{\tilde{U}_{\mathbf{w}}: \mathbf{w} \in \mathbb{Z}^{d}, \mathbf{w}(d)>0\right\}$, we construct the joint paths (as in Section 2) from the points $\mathbf{u}$ and $\mathbf{v}$ until its first simultaneous regeneration time $T_{1}(\mathbf{u}, \mathbf{v})$ of joint paths from $\mathbf{u}$ and $\mathbf{v}$.

Now, as defined in (19) , let $T_{1}^{(\text {Ind) }}(\mathbf{u}, \mathbf{v})$ be the first simultaneous regeneration time of two independent processes and $N_{1}^{\mathbf{u}}$ and $N_{1}^{\mathbf{v}}$ be the number of individual regenerations of the independent paths starting from $\mathbf{u}$ and $\mathbf{v}$ respectively. With the width of the explored region for two independent processes, as defined in (21), we consider the event where the total width of the explored region until the first simultaneous regeneration time of the two independent paths is less than $r$ (see Figure 51). More precisely, we consider the event

$$
A^{\text {Good }}(r):=\left\{W_{1}^{(\text {Ind })}(\mathbf{u}, \mathbf{v}) \leq r\right\} .
$$

On the event $A^{\text {Good }}(r)$ consider the following segments of paths:-

(i) the joint path process $\left(g_{n}(\mathbf{u}), g_{n}(\mathbf{v})\right)$ started simultaneously from $(\mathbf{u}, \mathbf{v})$, using 

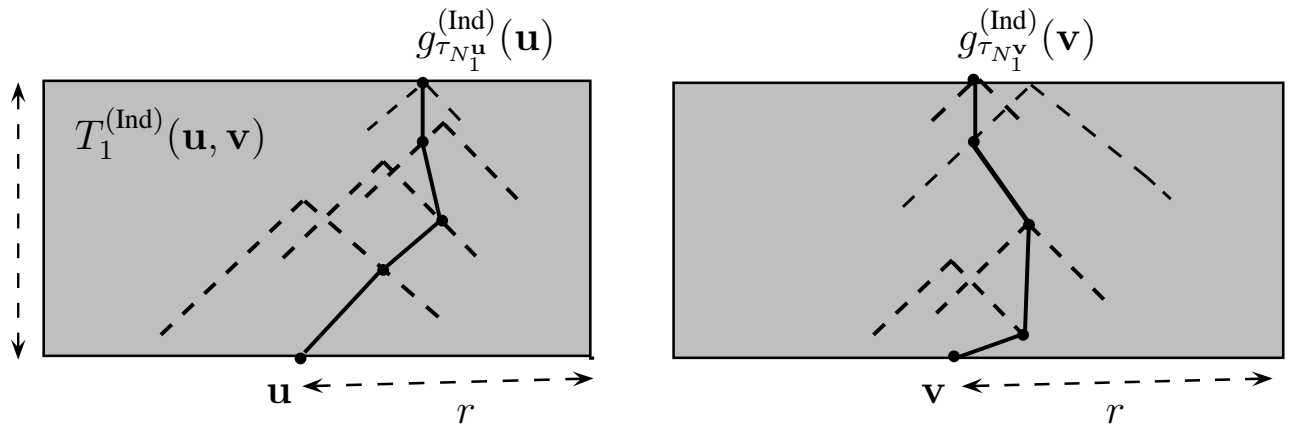

Figure 5: The shaded regions represent part of the cylinders (up to $T_{1}^{(\mathrm{Ind})}(\mathbf{u}, \mathbf{v})$ ) of width $r$ around $\mathbf{u}$ and $\mathbf{v}$. In the left cylinder we use the collection $\left\{U_{\mathbf{w}}^{\mathbf{u}}\right\}$, on the right cylinder we use the collection $\left\{U_{\mathbf{w}}^{\mathbf{v}}\right\}$ and in the remaining region, we use $\left\{U_{\mathbf{w}}\right\}$.

the collection $\left\{\tilde{U}_{\mathbf{w}}: \mathbf{w} \in \mathbb{Z}^{d}, \mathbf{w}(d)>0\right\}$, until the first simultaneous regeneration time $T_{1}(\mathbf{u}, \mathbf{v})$ of joint paths; and

(ii) the independent paths $\left\{g_{n}^{\text {(Ind) }}(\mathbf{u}): n \geq 0\right\}$, constructed using only the collection $\left\{U_{\mathbf{w}}^{\mathbf{u}}: \mathbf{w} \in \mathbb{Z}^{d}, \mathbf{w}(d)>0\right\}$, and $\left\{g_{n}^{\text {(Ind) }}(\mathbf{v}): n \geq 0\right\}$, constructed using only the collection $\left\{U_{\mathbf{w}}^{\mathbf{v}}: \mathbf{w} \in \mathbb{Z}^{d}, \mathbf{w}(d)>0\right\}$, until the first simultaneous regeneration of the independent paths $T_{1}^{(\text {Ind) }}(\mathbf{u}, \mathbf{v})$.

These two segments coincide as geometric paths (i.e. line segments in $\mathbb{R}^{2}$ ) although they may be indexed differently. Therefore, we must have

$$
T_{1}^{(\text {Ind })}(\mathbf{u}, \mathbf{v})=T_{1}(\mathbf{u}, \mathbf{v})
$$

and hence we have,

$$
\begin{aligned}
& \mathbb{P}\left[\left(\bar{g}_{\tau_{1}(\mathbf{u}, \mathbf{v})}(\mathbf{u}), \bar{g}_{\tau_{1}(\mathbf{u}, \mathbf{v})}(\mathbf{v})\right)=\left(\overline{\mathbf{u}}+\psi_{1}^{\mathbf{u}}, \overline{\mathbf{v}}+\psi_{1}^{\mathbf{v}}\right)\right] \\
& \geq \mathbb{P}\left(A^{\text {Good }}(r)\right) \\
& \geq 1-C_{15} \exp \left(-C_{16} r\right) .
\end{aligned}
$$

Finally, using the Markov property, we can use this coupling for each subsequent joint regeneration step. The new value of $d_{\min }$ for the $l$ th regeneration has to be computed from the position of the processes at the $l-1$ th joint regeneration and the value of $r$ has to be chosen accordingly.

\section{Trees and Forest}

In this section we prove Theorem 1.1. For $d=2,3$, we need to prove that for any $\mathbf{u}, \mathbf{v} \in V$, the paths $\pi^{\mathbf{u}}$ and $\pi^{\mathbf{v}}$ coincide eventually, i.e., $\pi^{\mathbf{u}}(t)=\pi^{\mathbf{v}}(t)$ for all $t \geq t_{0}$ for some $t_{0}<\infty$. 
First, we claim that it is enough to prove that $\pi^{\mathbf{u}}$ and $\pi^{\mathbf{v}}$ coincide eventually for $\mathbf{u}, \mathbf{v} \in V$ with $\mathbf{u}(d)=\mathbf{v}(d)$.

Indeed, for $\mathbf{u}, \mathbf{v} \in V$ with $\mathbf{u}(d)<\mathbf{v}(d)$ we have, from (25),

$$
\begin{gathered}
\mathbb{P}\left[\bigcap_{\mathbf{w} \in V, \mathbf{u}(d)=\mathbf{w}(d)}\left\{\text { the paths } \pi^{\mathbf{u}} \text { and } \pi^{\mathbf{w}} \text { coincide eventually }\right\}\right]=1 ; \\
\mathbb{P}\left[\bigcap_{\mathbf{w}^{\prime} \in V, \mathbf{w}^{\prime}(d)=\mathbf{v}(d)}\left\{\text { the paths } \pi^{\mathbf{v}} \text { and } \pi^{\mathbf{w}^{\prime}} \text { coincide eventually }\right\}\right]=1 .
\end{gathered}
$$

Further, $\mathbb{P}\left[\right.$ there exist $\mathbf{w}, \mathbf{w}^{\prime} \in V$ with $\left.\mathbf{w}(d)=\mathbf{u}(d), \mathbf{w}^{\prime}(d)=\mathbf{v}(d), h(\mathbf{w})=\mathbf{w}^{\prime}\right]=1$. Since, the intersection of these three events has probability $1, \pi^{\mathbf{u}}$ and $\pi^{\mathbf{v}}$ meet.

Now, to prove that for any two vertices $\mathbf{u}$ and $\mathbf{v}$ with $\mathbf{u}(d)=\mathbf{v}(d)$, the paths coincide eventually, we show that $\mathbb{P}\left(Z_{l}(\mathbf{u}, \mathbf{v})=0\right.$ for some $\left.l \geq 0\right)=1$ where $Z_{l}$ is as in (15). Recall, at the beginning of Section 3, we had observed that $\left\{Z_{l}(\mathbf{u}, \mathbf{v}): l \geq 0\right\}$ is a Markov chain taking values in $\mathbb{Z}^{d-1}$ with $\overline{\mathbf{0}} \in \mathbb{Z}^{d-1}$ being its only absorbing state.

\section{$4.1 \mathrm{~d}=2$}

Proposition 4.1. The paths of $\chi$ do not cross each other almost surely.

Proof: We present a proof based on Figure 6, a formal, though cumbersome, proof may be written on these lines.

Let $\mathbf{u}, \mathbf{v} \in \mathbb{Z}^{2}$ be as in Figure 6 with $\mathbf{u}(1) \leq \mathbf{v}(1)$ (otherwise we just interchange the roles of $\mathbf{u}$ and $\mathbf{v})$. We show that the edges $\langle\mathbf{u}, h(\mathbf{u})\rangle$ and $\langle\mathbf{v}, h(\mathbf{v})\rangle$ do not cross.

Let $S^{o}\left(\mathbf{u},\|\mathbf{u}-h(\mathbf{u})\|_{1}\right)=\left\{\mathbf{w} \in \mathbb{Z}:\|\mathbf{u}-\mathbf{w}\|_{1} \leq\|\mathbf{u}-h(\mathbf{u})\|_{1}\right.$ and $\left.\mathbf{w}(2) \geq \mathbf{u}(2)\right\}$ be the triangle $S^{+}\left(\mathbf{u},\|\mathbf{u}-h(\mathbf{u})\|_{1}\right)$ (defined at the beginning of Section 2) extended at the base by an extra set of edges on the horizontal line containing $\mathbf{u}$. Also let $\bar{S}^{o}(\mathbf{u}), \bar{S}^{+}(\mathbf{u}) \subseteq \mathbb{R}^{2}$ be the smallest simply connected closed triangle in $\mathbb{R}^{2}$ containing $S^{o}\left(\mathbf{u},\|\mathbf{u}-h(\mathbf{u})\|_{1}\right), S^{+}\left(\mathbf{u},\|\mathbf{u}-h(\mathbf{u})\|_{1}\right)$ respectively. The three linear segments of the triangle $\bar{S}^{o}(\mathbf{u})$ are appropriately called the base, the left boundary and the right boundary of it. Consider the case when $h(\mathbf{u})$ is a vertex on the left boundary of $\bar{S}^{+}(\mathbf{u})$. A similar argument may be given when $h(\mathbf{u})$ is a vertex on the right boundary of $\bar{S}^{+}(\mathbf{u})$.

In case $\bar{S}^{+}(\mathbf{u}) \cap \bar{S}^{+}(\mathbf{v})=\emptyset$, then $\langle\mathbf{u}, h(\mathbf{u})\rangle$ and $\langle\mathbf{v}, h(\mathbf{v})\rangle$ do not cross because they lie in $\bar{S}^{o}(\mathbf{u})$ and $\bar{S}^{o}(\mathbf{v})$ respectively.

On the other hand if $\bar{S}^{+}(\mathbf{u}) \cap \bar{S}^{+}(\mathbf{v}) \neq \emptyset$ then we note that -

(a) if the left boundary of $\bar{S}^{o}(\mathbf{u})$ has non-empty intersection with $\bar{S}^{o}(\mathbf{v})$, then, $h(\mathbf{u})$ being open, the left boundaries of $\bar{S}^{o}(\mathbf{u})$ and $\bar{S}^{o}(\mathbf{v})$ must have an overlap 


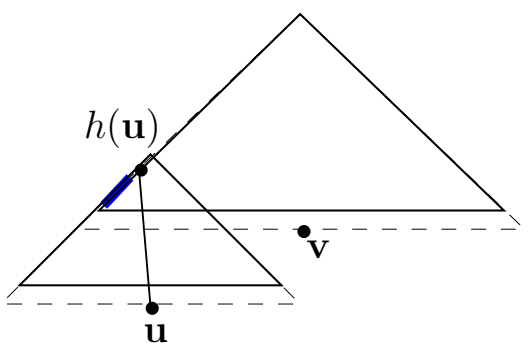

(a)

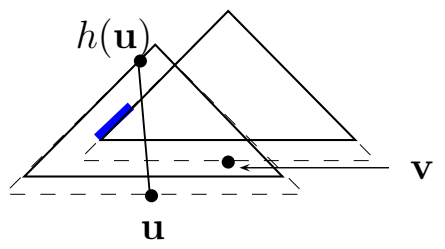

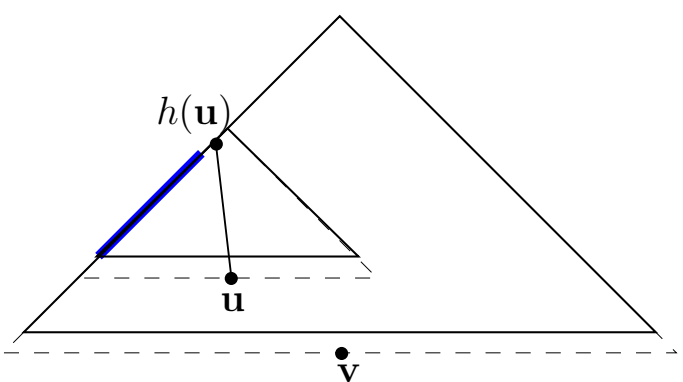

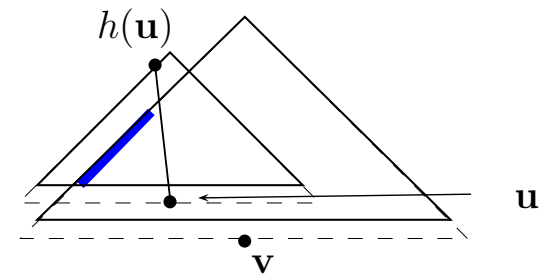

(b)

Figure 6: For the edges from $\mathbf{u}$ and $\mathbf{v}$ to cross, the location of $h(\mathbf{v})$ must be on the bold part of the appropriate triangle.

(see Figure 6 (a)). For the edges $\langle\mathbf{u}, h(\mathbf{u})\rangle$ and $\langle\mathbf{v}, h(\mathbf{v})\rangle$ to cross, $h(\mathbf{v})$ must necessarily be a lattice point, which is not $h(\mathbf{u})$, but lies on the interior of this overlap and below $h(\mathbf{u})$. Note that $h(\mathbf{v})$ being in the interior of this overlap violates the minimality of $U_{h(\mathbf{u})}$.

(b) if the left boundary of $\bar{S}^{\circ}(\mathbf{u})$ has empty intersection with $\bar{S}^{\circ}(\mathbf{v})$ (see Figure [6 (b)), then, for the edges $\langle\mathbf{u}, h(\mathbf{u})\rangle$ and $\langle\mathbf{v}, h(\mathbf{v})\rangle$ to cross, $h(\mathbf{v})$ must lie on the part of the left boundary of $\bar{S}^{o}(\mathbf{v})$ which is in the interior of $\bar{S}^{o}(\mathbf{u})$. However, this is not possible because the vertices in the interior of $\bar{S}^{o}(\mathbf{u})$ are closed.

This completes the proof of the proposition.

For the proof of Theorem 1.1 in the case $d=2$ we consider the process constructed from the two vertices $\mathbf{u}$ and $\mathbf{v}$ with $\mathbf{u}(2)=\mathbf{v}(2)$. Without loss of generality, we may assume that $\mathbf{u}(1)>\mathbf{v}(1)$. Since the paths $\left\{g_{n}(\mathbf{u}): n \geq 0\right\}$ and $\left\{g_{n}(\mathbf{v}): n \geq 0\right\}$ do not cross each other, from Corollary 3.1.1 we have that $\left\{Z_{l}(\mathbf{u}, \mathbf{v})=g_{\tau_{l}(\mathbf{u}, \mathbf{v})}(\mathbf{u})(1)-g_{\tau_{l}(\mathbf{u}, \mathbf{v})}(\mathbf{v})(1): l \geq 0\right\}$ is a non-negative martingale. By the martingale convergence theorem, there exists a random variable $Z_{\infty}$ such that $Z_{l}(\mathbf{u}, \mathbf{v}) \rightarrow Z_{\infty}$ a.s. as $l \rightarrow \infty$. Also, 0 being the only absorbing state of the Markov chain $\left\{Z_{l}(\mathbf{u}, \mathbf{v}): l \geq 0\right\}$ we have $Z_{\infty}=0$ a.s. and hence $Z_{l}(\mathbf{u}, \mathbf{v})=0$ for some $l$ a.s. This proves Theorem 1.1 for $d=2$. 


\section{$4.2 d=3$}

We show that Foster's criterion (see Asmussen [3], Proposition 5.3 of Chapter I, page 18), used in Gangopadhyay et al. [13, is applicable here. We start with the process constructed from the vertices $\mathbf{u}, \mathbf{v} \in \mathbb{Z}^{3}$ with $\mathbf{u}(3)=\mathbf{v}(3)$ and consider the process $Z_{l}=Z_{l}(\mathbf{u}, \mathbf{v})$ where $Z_{l}$ is as defined in (15). Also, changing the transition probability of $Z_{l}$ from the state $\mathbf{0}=(0,0)$ in any reasonable way, so that the state $\mathbf{0}$ is no longer absorbing, we make the Markov chain $\left\{Z_{l}(\mathbf{u}, \mathbf{v}): l \geq 0\right\}$ irreducible. With a slight abuse of notation, we continue to denote the modified chain by $\left\{Z_{l}(\mathbf{u}, \mathbf{v}): l \geq 0\right\}$ and it is enough to show that the modified chain is recurrent,

We now show that if the points are far apart, $Z_{l}(\mathbf{u}, \mathbf{v})$ can be approximated in expectation by the independent process.

Proposition 4.2. For any $\mathbf{u}, \mathbf{v}$ as above and $\mathrm{x} \in \mathbb{Z}^{2}$ and $m \geq 1$, we have

$$
\begin{aligned}
& \mid \mathbb{E}\left[\left(\left\|Z_{l+1}(\mathbf{u}, \mathbf{v})\right\|_{2}^{2}-\|\mathrm{x}\|_{2}^{2}\right)^{m} \mid Z_{l}(\mathbf{u}, \mathbf{v})=\mathrm{x}\right] \\
& -\mathbb{E}\left[\left(\left\|\left(\overline{\mathbf{u}}+\psi_{1}^{\mathbf{u}}\right)-\left(\overline{\mathbf{v}}+\psi_{1}^{\mathbf{v}}\right)\right\|_{2}^{2}-\|\mathrm{x}\|_{2}^{2}\right)^{m}\right] \mid \leq C_{17}^{(m)} \exp \left(-C_{18}^{(m)}\|\mathrm{x}\|_{2}\right)
\end{aligned}
$$

where $C_{17}^{(m)}$ and $C_{18}^{(m)}$ are positive constants, depending on $m$, and $\|\cdot\|_{2}$ denotes the standard Euclidean norm.

Proof: Since our model is spatially translation invariant and $Z_{l}(\mathbf{u}, \mathbf{v})$ is a time homogeneous Markov chain, we may take $\mathbf{v}=\mathbf{0}=(0,0,0)$ and $\mathbf{u}=(\mathrm{x}, 0)$ and $l=0$.

Now, we use the coupling described in Subsection 3.3, with $k=2$ and $r=$ $d_{\min } / 3=(|\mathrm{x}(1)|+|\mathrm{x}(2)|) / 3$. Observe that $\left\|Z_{1}(\mathbf{u}, \mathbf{v})-\mathrm{x}\right\|_{2} \leq\left\|Z_{1}(\mathbf{u}, \mathbf{v})-\mathrm{x}\right\|_{1} \leq$ $W_{1}(\mathbf{u}, \mathbf{v})$ and $\left\|\psi_{1}^{\mathbf{u}}-\psi_{1}^{\mathbf{v}}\right\|_{2} \leq\left\|\psi_{1}^{\mathbf{u}}-\psi_{1}^{\mathbf{v}}\right\|_{1} \leq W_{1}^{(\text {Ind })}(\mathbf{u}, \mathbf{v})$, where $W_{1}(\mathbf{u}, \mathbf{v})$ and $W_{1}^{\text {(Ind) }}(\mathbf{u}, \mathbf{v})$ are as defined in (9) and (21) respectively. Also, on the event $\left(A^{\text {Good }}(r)\right)^{c}$, we have $W_{1}(\mathbf{u}, \mathbf{v})>d_{\min } / 3=(|\mathrm{x}(1)|+|\mathrm{x}(2)|) / 3$ and $W_{1}^{(\mathrm{Ind})}(\mathbf{u}, \mathbf{v})>d_{\min } / 3=$ $(|\mathrm{x}(1)|+|\mathrm{x}(2)|) / 3$. Thus, with $\psi_{1}^{\mathbf{u}}$ and $\psi_{1}^{\mathbf{v}}$ as in (23), from the definition of $A^{\mathrm{Good}}(r)$ 
and equation (24), we have

$$
\begin{aligned}
& \mid \mathbb{E}\left[\left(\left\|Z_{1}(\mathbf{u}, \mathbf{v})\right\|_{2}^{2}-\|\mathrm{x}\|_{2}^{2}\right)^{m} \mid Z_{0}(\mathbf{u}, \mathbf{v})=\mathrm{x}\right] \\
& -\mathbb{E}\left[\left(\left\|\left(\overline{\mathbf{u}}+\psi_{1}^{\mathbf{u}}\right)-\left(\overline{\mathbf{v}}+\psi_{1}^{\mathbf{v}}\right)\right\|_{2}^{2}-\|\mathrm{x}\|_{2}^{2}\right)^{m}\right] \mid \\
& =\mid \mathbb{E}\left[\left(\left\|Z_{1}(\mathbf{u}, \mathbf{v})\right\|_{2}^{2}-\|\mathbf{x}\|_{2}^{2}\right)^{m} \mathbf{1}\left(\left(A^{\mathrm{Good}}(r)\right)^{c}\right) \mid Z_{0}(\mathbf{u}, \mathbf{v})=\mathrm{x}\right] \\
& -\mathbb{E}\left[\left(\left\|\left(\overline{\mathbf{u}}+\psi_{1}^{\mathbf{u}}\right)-\left(\overline{\mathbf{v}}+\psi_{1}^{\mathbf{v}}\right)\right\|_{2}^{2}-\|\mathrm{x}\|_{2}^{2}\right)^{m} \mathbf{1}\left(\left(A^{\mathrm{Good}}(r)\right)^{c}\right)\right] \mid \\
& \leq \mathbb{E}\left[2^{m}\left(\left\|Z_{1}(\mathbf{u}, \mathbf{v})\right\|_{2}^{2 m}+\|\mathrm{x}\|_{2}^{2 m}\right) \mathbf{1}\left(\left(A^{\operatorname{Good}}(r)\right)^{c}\right)\right] \\
& +\mathbb{E}\left[2^{m}\left(\left\|\left(\psi_{1}^{\mathbf{u}}-\psi_{1}^{\mathbf{v}}\right)+\mathrm{x}\right\|_{2}^{2 m}+\|\mathrm{x}\|_{2}^{2 m}\right) \mathbf{1}\left(\left(A^{\operatorname{Good}}(r)\right)^{c}\right)\right] \\
& \leq 2^{m} \mathbb{E}\left[\left(\|\mathrm{x}\|_{2}^{2 m}+2^{2 m}\left[\left\|Z_{1}(\mathbf{u}, \mathbf{v})-\mathbf{x}\right\|_{2}^{2 m}+\|\mathbf{x}\|_{2}^{2 m}\right]\right) \mathbf{1}\left(\left(A^{\text {Good }}(r)\right)^{c}\right)\right] \\
& +2^{m} \mathbb{E}\left[\left(\|\mathrm{x}\|_{2}^{2 m}+2^{2 m}\left[\left\|\psi_{1}^{\mathbf{u}}-\psi_{1}^{\mathbf{v}}\right\|_{2}^{2 m}+\|\mathbf{x}\|_{2}^{2 m}\right]\right) \mathbf{1}\left(\left(A^{\mathrm{Good}}(r)\right)^{c}\right)\right] \\
& \leq 2^{4 m}\left[\|\mathrm{x}\|_{2}^{2 m} \mathbb{P}\left(\left(A^{\text {Good }}(r)\right)^{c}\right)+\mathbb{E}\left[\left(W_{1}(\mathbf{u}, \mathbf{v})\right)^{2 m} \mathbf{1}\left(W_{1}(\mathbf{u}, \mathbf{v})>d_{\min } / 3\right)\right]\right. \\
& \left.+\mathbb{E}\left[\left(W_{1}^{(\mathrm{Ind})}(\mathbf{u}, \mathbf{v})\right)^{2 m} \mathbf{1}\left(W_{1}^{(\mathrm{Ind})}(\mathbf{u}, \mathbf{v})>d_{\min } / 3\right)\right]\right] \\
& \leq C_{17}^{(m)} \exp \left(-C_{18}^{(m)}\|\mathrm{x}\|_{2}\right)
\end{aligned}
$$

for a proper choice of $C_{17}^{(m)}, C_{18}^{(m)}>0$.

Using the properties of $\psi_{1}^{\mathbf{u}}$ and $\psi_{1}^{\mathbf{v}}$ from Proposition 3.4, we can compute the moments of $\left\|\left(\overline{\mathbf{u}}+\psi_{1}^{\mathbf{u}}\right)-\left(\overline{\mathbf{v}}+\psi_{1}^{\mathbf{v}}\right)\right\|_{2}^{2}-\|\mathrm{x}\|_{2}^{2}$. It is easy to check that

$$
\begin{aligned}
& \mathbb{E}\left[\left(\left\|\left(\overline{\mathbf{u}}+\psi_{1}^{\mathbf{u}}\right)-\left(\overline{\mathbf{v}}+\psi_{1}^{\mathbf{v}}\right)\right\|_{2}^{2}-\|\mathrm{x}\|_{2}^{2}\right)\right]=4 \mathbb{E}\left[\left(\psi_{1}^{\mathbf{u}}(1)\right)^{2}\right]=\alpha \text { (say) } \\
& \mathbb{E}\left[\left(\left\|\left(\overline{\mathbf{u}}+\psi_{1}^{\mathbf{u}}\right)-\left(\overline{\mathbf{v}}+\psi_{1}^{\mathbf{v}}\right)\right\|_{2}^{2}-\|\mathrm{x}\|_{2}^{2}\right)^{2}\right] \geq 8 \mathbb{E}\left[\left(\psi_{1}^{\mathbf{u}}(1)\right)^{2}\right]\|\mathrm{x}\|_{2}^{2}=2 \alpha\|\mathrm{x}\|_{2}^{2} ; \\
& \mathbb{E}\left[\left(\left\|\left(\overline{\mathbf{u}}+\psi_{1}^{\mathbf{u}}\right)-\left(\overline{\mathbf{v}}+\psi_{1}^{\mathbf{v}}\right)\right\|_{2}^{2}-\|\mathrm{x}\|_{2}^{2}\right)^{3}\right]=O\left(\|\mathrm{x}\|_{2}^{2}\right) .
\end{aligned}
$$

The proofs follow from straightforward calculations and have been relegated to the Appendix.

Now, we consider $f: \mathbb{Z}^{2} \rightarrow[0, \infty)$ defined by $f(\mathrm{x})=\sqrt{\log \left(1+\|\mathrm{x}\|_{2}^{2}\right)}$. Clearly, $f(\mathrm{x}) \rightarrow \infty$ as $\|\mathrm{x}\|_{2} \rightarrow \infty$. Using Taylor's expansion of the function $h(t)=$ $\sqrt{\log (1+t)}$ and observing that the fourth derivative of $h$ is always negative, we 
have

$$
\begin{aligned}
& \mathbb{E}\left[f\left(Z_{1}(\mathbf{u}, \mathbf{v})\right)-f\left(Z_{0}(\mathbf{u}, \mathbf{v})\right) \mid Z_{0}(\mathbf{u}, \mathbf{v})=\mathrm{x}\right] \\
& =\mathbb{E}\left[h\left(\left\|\left(Z_{1}(\mathbf{u}, \mathbf{v})\right)\right\|_{2}^{2}\right)-h\left(\left\|Z_{0}(\mathbf{u}, \mathbf{v})\right\|_{2}^{2}\right) \mid Z_{0}(\mathbf{u}, \mathbf{v})=\mathrm{x}\right] \\
& \leq \sum_{m=1}^{3} \frac{h^{(m)}\left(\|\mathrm{x}\|_{2}^{2}\right)}{m !} \mathbb{E}\left[\left(\left\|Z_{1}(\mathbf{u}, \mathbf{v})\right\|_{2}^{2}-\|\mathrm{x}\|_{2}^{2}\right)^{m} \mid Z_{0}(\mathbf{u}, \mathbf{v})=\mathrm{x}\right] \\
& \leq \sum_{m=1}^{3} \frac{h^{(m)}\left(\|\mathrm{x}\|_{2}^{2}\right)}{m !} \mathbb{E}\left[\left(\left\|\left(\overline{\mathbf{u}}+\psi_{1}^{\mathbf{u}}\right)-\left(\overline{\mathbf{v}}+\psi_{1}^{\mathbf{v}}\right)\right\|_{2}^{2}-\|\mathrm{x}\|_{2}^{2}\right)^{m}\right] \\
& \quad+\sum_{m=1}^{3} \frac{\left|h^{(m)}\left(\|\mathrm{x}\|_{2}^{2}\right)\right|}{m !} C_{17}^{(m)} \exp \left(-C_{18}^{(m)}\|\mathbf{x}\|_{2}\right)
\end{aligned}
$$

where $h^{(m)}$ represents the $m$ th derivative of $h$. Plugging in the expressions for $h^{(m)}$ and the moments given in (26) , (27) and (28), we have that the first sum above is bounded by $-\alpha\|\mathrm{x}\|_{2}^{2} /\left[8\left(1+\|\mathrm{x}\|_{2}^{2}\right)^{2}\left(\log \left(1+\left.\|\mathrm{x}\|\right|_{2} ^{2}\right)\right)^{3 / 2}\right]$ for all large $\|\mathrm{x}\|_{2}$ and the second sum is bounded by $C_{19} \exp \left(-C_{20}\|\mathrm{x}\|_{2}\right)$ for a proper choice of the constants $C_{19}$ and $C_{20}$. This yields that

$$
\mathbb{E}\left[f\left(Z_{l+1}(\mathbf{u}, \mathbf{v})\right)-f\left(Z_{l}(\mathbf{u}, \mathbf{v})\right) \mid Z_{l}(\mathbf{u}, \mathbf{v})=\mathrm{x}\right]<0
$$

for $\|\mathrm{x}\|_{2}$ large enough. This implies that $Z_{l}(\mathbf{u}, \mathbf{v})$ is recurrent and completes the proof for $d=3$.

\section{$4.3 d \geq 4$}

We present the proof for $d=4$; the argument being similar for $d>4$. To show that $\mathbb{P}(G$ has infinitely many distinct trees $)=1$, it is enough to prove that $\mathbb{P}(G$ has at least $m$ distinct trees $)=1$ for any $m \geq 2$. The probability measure $\mathbb{P}$ is ergodic as it is a product measure given by i.i.d. uniform random variables on each vertex of $\mathbb{Z}^{d}$. Clearly, for any $m \geq 2$, the event $\{G$ has at least $m$ distinct trees $\}$ is translation invariant under the group of translations and hence $\mathbb{P}(G$ has at least $m$ distinct trees $)$ is either 0 or 1 . So, it suffices to show that $\mathbb{P}(G$ has at least $m$ distinct trees) $>0$.

We first show the above for $m=2$. It is enough to exhibit two open vertices such that the paths from those two vertices do not meet with positive probability. We follow the same ideas as in [13] to achieve this; however there is one crucial change. In [13], each unit increase in the fourth axis represents an unit increase in time co-ordinate. For our model, the time taken for the joint regeneration of paths starting from two vertices is taken to be a unit of time. More precisely, starting with two open vertices $\mathbf{u}$ and $\mathbf{v}$ having the same fourth co-ordinate, at 
the first joint regeneration time of the paths from $\mathbf{u}$ and $\mathbf{v}$, we think of the time co-ordinate as having increased by a unit and at each joint regeneration thereafter, the time co-ordinate increases by one unit. At these joint regenerations, the fourth co-ordinates for both paths are equal. At the $l$ th regeneration, the paths have not yet met if $Z_{l}(\mathbf{u}, \mathbf{v}) \neq(0,0,0)$. Since the paths coalesce once they meet, it is enough to prove that with positive probability they do not meet at any of the joint regeneration times, i.e., $\mathbb{P}\left(Z_{l}(\mathbf{u}, \mathbf{v}) \neq(0,0,0)\right.$ for all $\left.l \geq 0\right)>0$ for some pair of open vertices $\mathbf{u}$ and $\mathbf{v}$.

Our strategy is to let the joint paths, from $\mathbf{u}$ and $\mathbf{v}$, evolve for $n^{4}$ joint regeneration times where $\mathbf{u}$ and $\mathbf{v}$ are sufficiently far apart (of order $n$ ). Then, with a very high probability the paths have travelled further away (of order $n^{2}$ ). Using the Markov property at the regeneration times, we may now start the paths from these new vertices and continue this process. We make this more precise in the following Proposition. For $\epsilon>0$, define the event

$$
A_{n, \epsilon}(\mathbf{u}, \mathbf{v}):=\left\{Z_{n^{4}}(\mathbf{u}, \mathbf{v}) \in D_{n^{2(1+\epsilon)}} \backslash D_{n^{2(1-\epsilon)}}\right\},
$$

where $D_{r}:=\left\{\mathbf{x} \in \mathbb{Z}^{3}:\|\mathbf{x}\|_{1} \leq r\right\}$. We show

Proposition 4.3. For $0<\epsilon<\frac{1}{3}$, there exist constants $C_{21}, \beta>0$ and $n_{0} \geq 1$ such that, for all $n \geq n_{0}$,

$$
\inf _{\overline{\mathbf{v}} \in \overline{\mathbf{u}}+D_{n^{1+\epsilon}} \backslash D_{n^{1-\epsilon}}} \mathbb{P}\left(A_{n, \epsilon}(\mathbf{u}, \mathbf{v}) \mid \mathbf{u}, \mathbf{v} \in V\right) \geq 1-C_{21} n^{-\beta} .
$$

Assuming Proposition 4.3, we first prove the result. Fix $\epsilon<1 / 3$ and choose $\mathbf{u}^{0}=(0,0,0,0)$ and $\mathbf{u}^{1}=\left(n_{0}, 0,0,0\right)$ where $n_{0}$ is as above. Let $E_{2}$ be the event that both $\mathbf{u}^{0}, \mathbf{u}^{1} \in V$, so that $\mathbb{P}\left(E_{2}\right)=p^{2}>0$. Clearly, $n_{0}^{1-\epsilon}<\| \mathbf{u}^{0}-$ $\mathbf{u}^{1} \|_{1}<n_{0}^{1+\epsilon}$. We show that $\mathbb{P}\left(Z_{l}\left(\mathbf{u}^{0}, \mathbf{u}^{1}\right) \neq(0,0,0)\right.$ for all $\left.l \geq 1 \mid E_{2}\right)>0$. For $j \geq 1$, set $r_{j}=\sum_{i=0}^{j-1}\left(n_{0}^{2^{i}}\right)^{4}$. Since $(0,0,0)$ is an absorbing state, we have $\mathbb{P}\left(Z_{l}\left(\mathbf{u}^{0}, \mathbf{u}^{1}\right) \neq(0,0,0)\right.$ for all $\left.l \geq 1 \mid E_{2}\right)=\lim _{j \rightarrow \infty} \mathbb{P}\left(Z_{r_{j}}\left(\mathbf{u}^{0}, \mathbf{u}^{1}\right) \neq(0,0,0) \mid\right.$

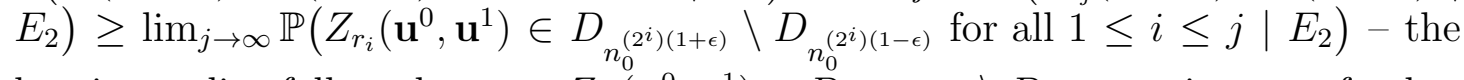
last inequality follows because $Z_{r_{i}}\left(\mathbf{u}^{0}, \mathbf{u}^{1}\right) \in D_{n_{0}^{\left(2^{i}\right)(1+\epsilon)}} \backslash D_{n_{0}^{\left(2^{i}\right)(1-\epsilon)}}$ imposes further restrictions on the Markov chain. For any $j \geq 1$, we have

$$
\begin{aligned}
\mathbb{P} & \left(Z_{r_{i}}\left(\mathbf{u}^{0}, \mathbf{u}^{1}\right) \in D_{n_{0}^{\left(2^{i}\right)(1+\epsilon)}} \backslash D_{n_{0}^{\left(2^{i}\right)(1-\epsilon)}} \text { for all } 1 \leq i \leq j \mid E_{2}\right) \\
= & \mathbb{P}\left(Z_{r_{j}}\left(\mathbf{u}^{0}, \mathbf{u}^{1}\right) \in D_{n_{0}^{\left(2^{j}\right)(1+\epsilon)}} \backslash D_{n_{0}^{\left(2^{j}\right)(1-\epsilon)}} \mid Z_{r_{j-1}}\left(\mathbf{u}^{0}, \mathbf{u}^{1}\right) \in D_{n_{0}^{\left(2^{j-1}\right)(1+\epsilon)}} \backslash D_{n_{0}^{\left(2^{j-1}\right)(1-\epsilon)}}\right) \\
\quad & \mathbb{P}\left(Z_{r_{j}}\left(\mathbf{u}^{0}, \mathbf{u}^{1}\right) \in D_{n_{0}^{\left(2^{i}\right)(1+\epsilon)}} \backslash D_{n_{0}^{\left(2^{i}\right)(1-\epsilon)}} \text { for all } 1 \leq i \leq j-1 \mid E_{2}\right)
\end{aligned}
$$

Now, using the Markov property of $Z_{l}\left(\mathbf{u}^{0}, \mathbf{u}^{1}\right)$, Proposition 4.3 and the translation invariance of our model, we see that the conditional probability in the second line 


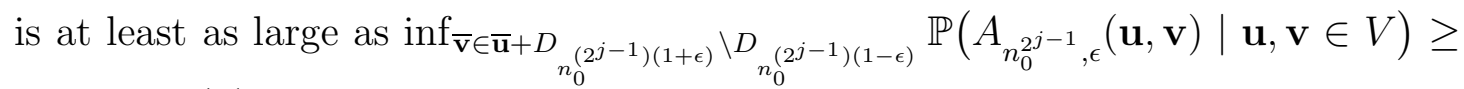
$1-C_{21}\left(n_{0}^{2^{j-1}}\right)^{-\beta}$. By repeating this argument, we conclude that

$$
\mathbb{P}\left(Z_{r_{j}}\left(\mathbf{u}^{0}, \mathbf{u}^{1}\right) \neq(0,0,0)\right) \geq \prod_{i=1}^{j}\left[1-C_{21}\left(n_{0}^{2^{i-1}}\right)^{-\beta}\right] \rightarrow \prod_{i=1}^{\infty}\left[1-C_{21}\left(n_{0}^{2^{i-1}}\right)^{-\beta}\right] .
$$

Therefore, $\mathbb{P}(G$ has at least 2 distinct trees $) \geq p^{2} \prod_{i=1}^{\infty}\left[1-C_{21}\left(n_{0}^{2^{i-1}}\right)^{-\beta}\right]>0$.

The above calculations hold for any pair of points which satisfy the initial condition. We now use this to prove that $\mathbb{P}(G$ has at least $m$ distinct trees $)>0$ for any $m \geq 2$. Fix $\delta>0$ such that $m(m-1) \delta / 2<1$. Note that $\prod_{i=1}^{\infty}[1-$ $\left.C_{21}\left(n^{2^{i-1}}\right)^{-\beta}\right] \rightarrow 1$ as $n \rightarrow \infty$. Now, we choose $n_{1}>n_{0}$ so large that $\prod_{i=1}^{\infty}[1-$ $\left.C_{21}\left(n_{1}^{2^{i-1}}\right)^{-\beta}\right]>1-\delta$ and $m<n_{1}^{\epsilon}$ where $\epsilon$ and $n_{0}$ are as above. Now, consider the points $\mathbf{u}^{i}=\left((i-1) n_{1}, 0,0,0\right)$ for $i=1,2, \ldots, m$. Clearly, all of them have the same fourth co-ordinate and $n_{1}^{1-\epsilon}<n_{1} \leq\left\|\mathbf{u}^{i}-\mathbf{u}^{j}\right\|_{1} \leq m n_{1}<n_{1}^{1+\epsilon}$. Let $E_{m}$ be the event that all the points $\mathbf{u}^{1}, \ldots, \mathbf{u}^{m}$ are open. Then $\mathbb{P}\left(E_{m}\right)=p^{m}>0$. We now consider the event $A_{i, j}$ that the paths from $\mathbf{u}^{i}$ and $\mathbf{u}^{j}$ do not meet for $i>j$. From above calculations and by our choice of $n_{1}$, we have $\mathbb{P}\left(A_{i, j} \mid E_{m}\right)>1-\delta$. Further, we consider the intersection of all the events $A_{i, j}$ for $1 \leq j<i \leq m$. Clearly, $\mathbb{P}\left(\cap_{1 \leq j<i \leq m} A_{i, j} \mid E_{m}\right) \geq 1-m(m-1) \delta / 2$ so that $\mathbb{P}(G$ has at least $m$ distinct trees $) \geq p^{m}(1-m(m-1) \delta / 2)>0$.

To prove Proposition 4.3, we define a new event where paths are constructed by using independent uniform random variables of their own and then use the coupling described in Subsection 3.3. Consider the event

$$
\begin{aligned}
& A_{n, \epsilon}^{(\operatorname{Ind})}(\mathbf{u}, \mathbf{v}):=\left\{\overline{\mathbf{v}}+\sum_{l=1}^{n^{4}} \psi_{l}^{\mathbf{v}} \in \overline{\mathbf{u}}+\sum_{l=1}^{n^{4}} \psi_{l}^{\mathbf{u}}+D_{n^{2(1+\epsilon)}} \backslash D_{n^{2(1-\epsilon)}},\right.
\end{aligned}
$$

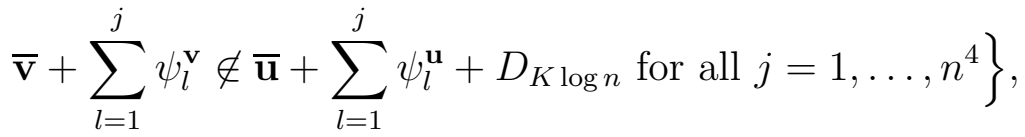

where $K$ is a suitably chosen large constant. We have

Proposition 4.4. For $0<\epsilon<\frac{1}{3}$, there exists $n_{0}$ such that

$$
\inf _{\overline{\mathbf{v}} \in \overline{\mathbf{u}}+D_{n^{1+\epsilon}} \backslash D_{n^{1-\epsilon}}} \mathbb{P}\left(A_{n, \epsilon}^{(\mathrm{Ind})}(\mathbf{u}, \mathbf{v})\right) \geq 1-C_{22} n^{-\alpha},
$$

for some constant $C_{22}, \alpha>0$ and for all $n \geq n_{0}$.

First we prove Proposition 4.3 assuming the result of Proposition 4.4 .

Proof of Proposition 4.3. We employ the coupling described earlier in Subsection 3.3 , on the event $A_{n, \epsilon}^{(\text {Ind })}(\mathbf{u}, \mathbf{v})$ defined above. This time we will continue 
the coupling step by step for $n^{4}$ simultaneous regeneration steps of independent paths. At each step we choose $r=K \log n / 3$ and say that the coupling is successful at step $j$ if the event $A^{\text {Good }}(r)$ occurs. We do the coupling at step $j+1$ if the coupling is successful at step $j$. Note, if the coupling is successful at every step $j=1, \ldots, n^{4}$, we have, for $j=1,2, \ldots, n^{4}$,

$$
\overline{\mathbf{u}^{0}}+\sum_{l=1}^{j} \psi_{l}^{\mathbf{u}^{0}}=\bar{g}_{\tau_{j}\left(\mathbf{u}^{0}, \mathbf{v}^{0}\right)}\left(\mathbf{u}^{0}\right) \text { and } \overline{\mathbf{v}^{0}}+\sum_{l=1}^{j} \psi_{l}^{\mathbf{v}^{0}}=\bar{g}_{\tau_{j}\left(\mathbf{u}^{0}, \mathbf{v}^{0}\right)}\left(\mathbf{v}^{0}\right) .
$$

Therefore, we get

$\mathbb{P}\left(A_{n, \epsilon}\left(\mathbf{u}^{0}, \mathbf{v}^{0}\right)\right) \geq \mathbb{P}\left(A_{n, \epsilon}^{(\mathrm{Ind})}\left(\mathbf{u}^{0}, \mathbf{v}^{0}\right) \cap\left\{\right.\right.$ Coupling is successful for $\left.\left.j=1,2, \ldots, n^{4}\right\}\right)$.

Using the Markov property and the estimate of the coupling being successful, given in (24), we obtain, for all sufficiently large $n$,

$$
\mathbb{P}\left(A_{n, \epsilon}\left(\mathbf{u}^{0}, \mathbf{v}^{0}\right)\right) \geq 1-C_{22} n^{-\alpha}-C_{15} n^{4} \exp \left(-C_{16} K \log n / 3\right) \geq 1-C_{21} n^{-\beta}
$$

for suitable choice of $\beta>0$ and $C_{21}$. This proves the proposition 4.3.

Finally, we indicate the steps for proving Proposition 4.4. By Remark 3.5, $\left\{S_{j}=\overline{\mathbf{v}}-\overline{\mathbf{u}}+\sum_{l=1}^{j} \psi_{l}^{\mathbf{v}}-\psi_{l}^{\mathbf{u}}: j \geq 1\right\}$ is an aperiodic, isotropic, symmetric random walk on $\mathbb{Z}^{3}$ starting from $\overline{\mathbf{v}}-\overline{\mathbf{u}}$. The event $\mathbb{P}\left(A_{n, \epsilon}\left(\mathbf{u}^{0}, \mathbf{v}^{0}\right)\right)$ is not satisfied if any of the following occurs : (a) the random walk travels too far, i.e., $\left\{S_{n^{4}} \notin D_{n^{2(1+\epsilon)}}\right\}$ or the random walk travels too little, i.e., $\left\{S_{n^{4}} \in D_{n^{2(1-\epsilon)}}\right)$ or (c) it comes too close to a given point at distance of order $n$, i.e., $\left\{S_{j} \in-(\overline{\mathbf{v}}-\overline{\mathbf{u}})+D_{K \log n}\right.$, for some $\left.1 \leq j \leq n^{4}\right\}$. For an aperiodic, isotropic, symmetric random walk, it can be shown that each of these events have small probability. For more details, we refer the reader to Lemma 3.3 of [13].

\section{Brownian Web}

In this section we prove Theorem 1.2 . We begin by recalling that the Brownian web takes values in the metric space $\mathcal{H}$ equipped with the Hausdorff metric $d_{\mathcal{H}}$ where $\mathcal{H}$ is the space of compact subsets of the path space ( $\Pi, d_{\Pi}$ ) (see the discussion in the paragraphs after the statement of Theorem 1.1 in Section 1). As introduced earlier, for any $n \geq 1$, the collection of scaled paths $\mathcal{X}_{n}(\gamma, \sigma)$ is obtained from $G$ with normalization constants $\gamma, \sigma$ and we had remarked that the closure of $\mathcal{X}_{n}(\gamma, \sigma)$ in $\left(\Pi, d_{\Pi}\right)$ denoted by $\overline{\mathcal{X}}_{n}(\gamma, \sigma)$ is a $\left(\mathcal{H}, \mathcal{B}_{\mathcal{H}}\right)$ valued random variable.

We need some more notation. For a subset $\Gamma \subseteq \Pi$ of paths and for $t \in \mathbb{R}$ let $\Gamma^{t}:=\left\{\pi \in \Gamma: \sigma_{\pi} \leq t\right\}$ be the set of paths which start 'below' $t$. For $t>0$ and 
$t_{0}, a, b \in \mathbb{R}$ with $a<b$, we define two counting random variables as follows

$$
\begin{aligned}
& \eta_{\Gamma}\left(t_{0}, t ; a, b\right):=\#\left\{\pi\left(t_{0}+t\right): \pi \in \Gamma^{t_{0}} \text { and } \pi\left(t_{0}\right) \in[a, b]\right\} \text { and } \\
& \hat{\eta}_{\Gamma}\left(t_{0}, t ; a, b\right):=\#\left\{\pi\left(t_{0}+t\right): \pi \in \Gamma^{t_{0}} \text { and } \pi\left(t_{0}+t\right) \in[a, b]\right\} .
\end{aligned}
$$

Theorem 2.2 in Fontes et al. [11] provided a criteria for a sequence of $\left(\mathcal{H}, \mathcal{B}_{\mathcal{H}}\right)$ valued random variables with non-crossing paths to converge weakly to the Brownian web. In the following we denote, the standard Brownian motion starting from $\mathbf{x}$ by $B^{\mathbf{x}}$ and standard coalescing Brownian motions starting from $\mathbf{x}^{1}, \ldots, \mathbf{x}^{k}$ respectively, by $\left(W^{\mathbf{x}^{1}}, \ldots, W^{\mathbf{x}^{k}}\right)$.

Theorem 5.1. [11] Suppose $\xi_{1}, \xi_{2}, \ldots$ are $\left(\mathcal{H}, B_{\mathcal{H}}\right)$ valued random variables with non-crossing paths. Assume that the following conditions hold.

$\left(I_{1}\right)$ For all $\mathbf{y} \in \mathbb{R}^{2}$, there exist $\zeta_{n}^{\mathbf{y}} \in \xi_{n}$ such that for any finite set of points $\mathbf{x}^{1}, \ldots, \mathbf{x}^{k}$ from a deterministic countable dense set $\mathcal{D}$ of $\mathbb{R}^{2}$, as $n \rightarrow \infty$, $\left(\zeta_{n}^{\mathbf{x}^{1}}, \ldots, \zeta_{n}^{\mathbf{x}^{k}}\right) \Rightarrow\left(W^{\mathbf{x}^{1}}, \ldots, W^{\mathbf{x}^{k}}\right)$.

$\left(B_{1}\right)$ For all $t>0, \lim \sup _{n \rightarrow \infty} \sup _{\left(a, t_{0}\right) \in \mathbb{R}^{2}} \mathbb{P}\left(\eta_{\xi_{n}}\left(t_{0}, t ; a, a+\epsilon\right) \geq 2\right) \rightarrow 0$ as $\epsilon \downarrow 0$.

$\left(B_{2}\right)$ For all $t>0, \frac{1}{\epsilon} \lim \sup _{n \rightarrow \infty} \sup _{\left(a, t_{0}\right) \in \mathbb{R}^{2}} \mathbb{P}\left(\eta_{\xi_{n}}\left(t_{0}, t ; a, a+\epsilon\right) \geq 3\right) \rightarrow 0$ as $\epsilon \downarrow 0$.

Then $\xi_{n}$ converges in distribution to the standard Brownian web $\mathcal{W}$.

The convergence in $\left(I_{1}\right)$ occurs in the space $\Pi^{k}$. Note that the convergence in $\Pi$ implies that the starting points converge as points in $\mathbb{R}^{2}$ and the paths converge uniformly on the compact sets of time.

In Theorem 1.4 and Lemma 6.1 of Newman et al. [17], it was further proved that the condition $\left(B_{2}\right)$ can be replaced by $\left(E_{1}^{\prime}\right)$ where

$\left(E_{1}^{\prime}\right)$ if $\mathcal{Z}^{t_{0}}$ is any subsequential limit of $\left\{\mathcal{X}_{n}^{t_{0}}: n \geq 1\right\}$ for $t_{0} \in \mathbb{R}$, then for all $t, a, b \in \mathbb{R}$ with $t>0$ and $a<b, \mathbb{E}\left[\hat{\eta}_{\mathcal{Z}^{t_{0}}}\left(t_{0}, t ; a, b\right)\right] \leq \mathbb{E}\left[\hat{\eta}_{\mathcal{W}}\left(t_{0}, t ; a, b\right)\right]=\frac{b-a}{\sqrt{t \pi}}$.

It is worthwhile mentioning here that for a sequence of $\left(\mathcal{H}, \mathcal{B}_{\mathcal{H}}\right)$ valued random variables $\xi_{n}$ with non-crossing paths, property $\left(I_{1}\right)$ implies tightness (see Proposition B.2 in the Appendix of [11]) and hence such a subsequential limit $\mathcal{Z}^{t_{0}}$ exists. Thus, to prove Theorem 1.2 we need to show that for some $\gamma(p)>0$ and $\sigma(p)>0$ the sequence $\overline{\mathcal{X}}_{n}(\gamma, \sigma)$ satisfies the conditions $\left(I_{1}\right),\left(B_{1}\right)$ and $\left(E_{1}^{\prime}\right)$ and hence converges to the standard Brownian web. 


\subsection{Verification of condition $\left(I_{1}\right)$}

We proceed by the method of induction and follow a mixture of argument of Ferrari et al. [10] and Coletti et al. [7]. In Section 2, we proved a regeneration property for any single path, which we use in Proposition 5.2 to show the convergence of this path (appropriately scaled) to a Brownian motion. For showing the joint convergence of more than one path, we use the fact that the paths behave (almost) independently when they are separated by a large distance and, when they come close to each other, they coalesce very quickly. This idea was initially introduced by Ferrari et al. [10]. It should be noted here that the dependency structure of our model is quite different from that of [10] where paths are independent when they are separated by a fixed distance. Later Coletti et al. [7] modified it to deal with long range interactions and we use a similar approach to prove the joint convergence of paths.

We first recall that for a path $\pi^{\mathbf{u}}$ and $\gamma, \sigma>0$, the scaled path is defined by $\pi_{n}^{\mathbf{u}}=\pi_{n}^{\mathbf{u}}(\gamma, \sigma):\left[\mathbf{u}(2) / n^{2} \gamma, \infty\right] \rightarrow[-\infty, \infty]$ such that $\pi_{n}^{\mathbf{u}}(t)=\pi\left(n^{2} \gamma t\right) / n \sigma$. Note that the distribution of the path $\pi^{\mathbf{u}}$ depends only on uniform random variables in $\{y>\mathbf{u}(2)\}$ and is independent of the open/closed status of $\mathbf{u}$. We first show that the scaled path starting from the origin converges to the standard Brownian motion.

Proposition 5.2. There exist $\gamma:=\gamma(p)$ and $\sigma:=\sigma(p)$ such that as $n \rightarrow \infty$,

$$
\pi_{n}^{\mathbf{0}} \Rightarrow B^{\mathbf{0}} \text { in }\left(\Pi, d_{\Pi}\right) .
$$

Proof: We use the fact that the path in between the regeneration steps can be broken up into i.i.d. pieces. For a path $\pi$ we define the modified path $\tilde{\pi}$ which is linear between successive regeneration points of $\pi$. Using Proposition 2.8 we have that the displacements between successive regeneration times are independent and have exponential moments - this allows an application of Donsker's invariance principle to the modified path to prove the convergence to the Brownian motion.

Let $\tau_{j}$ and $T_{j}$ denote the $j$ th regeneration step and time respectively for the path starting from $\mathbf{0}$ (see (11) and (10)). Remembering that $g_{j}(\mathbf{0})$ is the position of the path starting from $\mathbf{0}$ after the $j$ th step, let $Y_{j}=Y_{j}^{(\mathbf{0})}=\bar{g}_{\tau_{j}}(\mathbf{0})-\bar{g}_{\tau_{j-1}}(\mathbf{0})=$ $g_{\tau_{j}}(\mathbf{0})(1)-g_{\tau_{j-1}}(\mathbf{0})(1)($ see $(13))$. We define a piecewise linear path $\tilde{\pi}$ as follows: for $T_{j} \leq t<T_{j+1}, j \geq 0$,

$$
\tilde{\pi}(t):=g_{\tau_{j}}(\mathbf{0})(1)+\frac{t-T_{j}}{T_{j+1}-T_{j}}\left(g_{\tau_{j+1}}(\mathbf{0})(1)-g_{\tau_{j}}(\mathbf{0})(1)\right)
$$

and its diffusively scaled version $\tilde{\pi}_{n}$ by

$$
\tilde{\pi}_{n}(t)=\tilde{\pi}_{n}(\gamma, \sigma)(t):=\frac{1}{n \sigma} \tilde{\pi}\left(n^{2} \gamma t\right)
$$


for $t \geq 0$. Next we define another stochastic process, $S$ on $[0, \infty)$ as follows: for $j \leq t<j+1, j \geq 0$,

$$
S(t)=T_{j}+(t-j)\left(T_{j+1}-T_{j}\right) .
$$

Clearly, $S(t)$ is a strictly increasing process. Hence, $t \mapsto S(t)$ admits an inverse $S^{-1}(t)$ which is also strictly increasing. The process $S(t)$ denotes the time change required to track the path $\tilde{\pi}$. More precisely, we have, for $t \geq 0$,

$$
\tilde{\pi}_{n}(t)=X_{n}\left(S^{-1}\left(n^{2} \gamma t\right) / n^{2}\right) .
$$

where the process $X_{n}=X_{n}(\gamma, \sigma)$ on $[0, \infty)$ is defined as follows: $X_{n}(0)=0$ and for $t>0$,

$$
X_{n}(t):=\frac{1}{n \sigma}\left[\left(n^{2} \gamma t-\left\lfloor n^{2} \gamma t\right\rfloor\right) Y_{\left\lfloor n^{2} \gamma t\right\rfloor+1}+\sum_{i=1}^{\left\lfloor n^{2} \gamma t\right\rfloor} Y_{i}\right] .
$$

From Remark 2.9, $Y_{i}$ 's are symmetric and i.i.d., so that $\mathbb{E}\left(Y_{1}\right)=0$. Thus, from Donsker's invariance principle, it follows that, for

$$
\sigma=\sigma_{0}:=\sqrt{\operatorname{Var}\left(Y_{1}\right)}
$$

the process $X_{n}$ converges weakly to the standard Brownian motion starting from 0 .

Let $N(t)$ be the number of the renewals for the process $S(t)$ up to time $t$, i.e., $N(t)=\left\lfloor S^{-1}(t)\right\rfloor$ so that, $N(t) \leq S^{-1}(t) \leq N(t)+1$. By the renewal theorem (see Theorem 4.4.1 of Durrett [9]), $S^{-1}\left(n^{2} \gamma t\right) / n^{2} \rightarrow g(t):=\frac{\gamma t}{\mathbb{E}\left(T_{1}\right)}, t \geq 0$ almost surely. Taking

$$
\gamma=\gamma_{0}:=\mathbb{E}\left[T_{1}\right]
$$

we conclude that

$$
\tilde{\pi}_{n} \Rightarrow B^{0}
$$

Finally to conclude the result, it is enough to show that, for any $s>0$ and $\epsilon>0$

$$
\begin{aligned}
& \mathbb{P}\left[\sup \left\{\left|\tilde{\pi}_{n}(t)-\pi_{n}^{\mathbf{0}}(t)\right|: t \in[0, s]\right\}>\epsilon\right] \\
= & \mathbb{P}\left[\sup \left\{\left|\tilde{\pi}(t)-\pi^{\mathbf{0}}(t)\right|: t \in\left[0, n^{2} \gamma s\right]\right\}>n \sigma \epsilon\right] \rightarrow 0
\end{aligned}
$$

as $n \rightarrow \infty$. From the definition of $W_{j}(\mathbf{0})$ (see (9)), for any $t \in\left[T_{j}, T_{j+1}\right]$ we have $\left|\tilde{\pi}(t)-\pi^{\mathbf{0}}(t)\right| \leq 2 W_{j+1}$ for all $j \geq 0$. Since $N\left(n^{2} \gamma s\right) \leq\left\lfloor n^{2} \gamma s\right\rfloor$, we have

$$
\begin{aligned}
& \mathbb{P}\left[\sup \left\{\left|\tilde{\pi}(t)-\pi^{\mathbf{0}}(t)\right|: t \in\left[0, n^{2} \gamma s\right]\right\}>n \sigma \epsilon\right] \\
\leq & \mathbb{P}\left[2 \max \left\{W_{j}(\mathbf{0}): j=1, \ldots,\left\lfloor n^{2} \gamma s\right\rfloor\right\}>n \sigma \epsilon\right] \\
\leq & \left\lfloor n^{2} \gamma s\right\rfloor \mathbb{P}\left(2 W_{1}(\mathbf{0})>n \sigma \epsilon\right) \rightarrow 0 \text { as } n \rightarrow \infty,
\end{aligned}
$$


where the last step follows from Proposition 2.8, This completes the proof.

Henceforth, we assume that we are working with $\gamma=\gamma_{0}$ and $\sigma=\sigma_{0}$ and for the ease of writing we drop $(\gamma, \sigma)$ from our notation unless required.

By translation invariance of our model, we have

$$
\left\{g_{m}\left(\mathbf{u}_{n}\right): m \geq 0\right\} \stackrel{d}{=} \mathbf{u}_{n}+\left\{g_{m}(\mathbf{0}): m \geq 0\right\} .
$$

Using Proposition 5.2, we conclude the following corollary:

Corollary 5.2.1. For any $\mathbf{u} \in \mathbb{R}^{2}$ and a sequence $\left\{\mathbf{u}_{n} \in \mathbb{Z}^{2}: n \geq 1\right\}$ such that $\left(\mathbf{u}_{n}(1) / n \sigma, \mathbf{u}_{n}(2) / n^{2} \gamma\right) \rightarrow \mathbf{u}$ as $n \rightarrow \infty$, we have

$$
\pi_{n}^{\mathbf{u}_{n}} \Rightarrow B^{\mathbf{u}} \text { in }\left(\Pi, d_{\pi}\right)
$$

We now show that, if two paths start close to each other on the $\mathrm{x}$-axis, they converge to the same Brownian motion.

Proposition 5.3. Let $\mathbf{u}_{n}=\left(u_{n}, 0\right), \mathbf{v}_{n}=\left(v_{n}, 0\right) \in \mathbb{Z}^{2}$ be such that $u_{n}<0<v_{n}$ and $\left(v_{n}-u_{n}\right) / n \rightarrow 0$. Then,

$$
\left(\pi_{n}^{\mathbf{u}_{n}}, \pi_{n}^{\mathbf{v}_{n}}\right) \Rightarrow\left(B^{\mathbf{0}}, B^{\mathbf{0}}\right) .
$$

Proof: By Corollary 5.2.1, $\pi_{n}^{\mathbf{u}_{n}} \Rightarrow B^{\mathbf{0}}$ and $\pi_{n}^{\mathbf{v}_{n}} \Rightarrow B^{\mathbf{0}}$. Therefore, $\left\{\pi_{n}^{\mathbf{u}_{n}}: n \geq 1\right\}$ and $\left\{\pi_{n}^{\mathbf{v}_{n}}: n \geq 1\right\}$ are both tight in $\left(\Pi, d_{\Pi}\right)$, and hence $\left\{\left(\pi_{n}^{\mathbf{u}_{n}}, \pi_{n}^{\mathbf{v}_{n}}\right): n \geq 1\right\}$ is tight in the product space. Now, consider any convergent subsequence and assume that $(B, \tilde{B})$ is the subsequential limit. Since $\pi_{n}^{\mathbf{u}_{n}} \Rightarrow B^{\mathbf{0}}$ and $\pi_{n}^{\mathbf{v}_{n}} \Rightarrow B^{\mathbf{0}}$, both $B$ and $\tilde{B}$ are standard Brownian motions starting from the origin. Using Skorohod's representation theorem, we can couple so that the convergence is almost sure. Furthermore, by the non-crossing property of the path family, $\pi_{n}^{\mathbf{u}_{n}}(s) \leq \pi_{n}^{\mathbf{v}_{n}}(s)$ for all $s \geq 0$. Hence, we have that $B(s) \leq \tilde{B}(s)$ for all $s \geq 0$. This implies that $B(s)=\tilde{B}(s)$ for all $s \geq 0$ almost surely.

For verifying condition $\left(I_{1}\right)$, we require an estimate on the displacements of paths in the presence of some information. Our next proposition estimates the distance traversed by a path (either laterally or vertically) in terms of the height of the region whose information is known.

Proposition 5.4. Let $\mathcal{R} \subseteq \mathbb{H}\left(m^{\beta}\right)$ for some $m \geq 1$ and $0<\beta<1 / 2$. For the path $\pi^{\mathbf{0}}$ staring from the origin $\mathbf{0}=(0,0)$, given any configuration on $\mathcal{R}$ and any $\delta>2 \beta$ we have

$$
\mathbb{P}\left(\sup \left\{\left|\pi^{\mathbf{0}}(s)\right|: 0 \leq s \leq m^{\beta}\right\} \geq m^{\delta} \mid\left\{U_{\mathbf{w}}: \mathbf{w} \in \mathcal{R}\right\}\right) \leq C_{23} m^{\beta} \exp \left(-C_{24} m^{\beta}\right)
$$

where $C_{23}$ and $C_{24}$ are positive constants. 
Proof: Consider the horizontal line $\left\{y=\left\lfloor m^{\beta}\right\rfloor+1\right\}$ lying above the region $\mathcal{R}$. For the construction of the path $\pi^{\mathbf{0}}$ there is no information regarding the configuration on the lattice points on or above this line. Consider the following events:

$$
\begin{aligned}
& E_{m}^{(1)}:=\bigcap_{i=1}^{\left\lfloor m^{\beta}\right\rfloor+1} \bigcup_{u=2(i-1)\left\lfloor m^{\beta}\right\rfloor+1}^{(2 i-1)\left\lfloor m^{\beta}\right\rfloor}\left\{\left(u,\left\lfloor m^{\beta}\right\rfloor+1\right) \in V\right\} ; \\
& E_{m}^{(2)}:=\bigcap_{i=1}^{\left\lfloor m^{\beta}\right\rfloor+1} \bigcup_{u=-(2 i-1)\left\lfloor m^{\beta}\right\rfloor}^{-2(i-1)\left\lfloor m^{\beta}\right\rfloor-1}\left\{\left(u,\left\lfloor m^{\beta}\right\rfloor+1\right) \in V\right\} .
\end{aligned}
$$

On the event $E_{m}^{(1)}\left(E_{m}^{(2)}\right)$, there are one or more open vertices in each of the blocks $\left[2(i-1)\left\lfloor m^{\beta}\right\rfloor+1,(2 i-1)\left\lfloor m^{\beta}\right\rfloor\right] \times\left\{\left\lfloor m^{\beta}\right\rfloor+1\right\}$ (respectively from $[-(2 i-$ 1) $\left.\left.\left\lfloor m^{\beta}\right\rfloor,-2(i-1)\left\lfloor m^{\beta}\right\rfloor-1\right] \times\left\{\left\lfloor m^{\beta}\right\rfloor+1\right\}\right)$ of size $\left\lfloor m^{\beta}\right\rfloor$ for $i=1,2, \ldots,\left\lfloor m^{\beta}\right\rfloor+1$. Clearly, $E_{m}:=E_{m}^{(1)} \cap E_{m}^{(2)}$ depends only on the uniform random variables on $\left\{y=\left\lfloor m^{\beta}\right\rfloor+1\right\}$ and hence, is independent of the history. Further, $\mathbb{P}\left(E_{m}^{(1)}\right)=$ $\mathbb{P}\left(E_{m}^{(2)}\right)=\left(1-(1-p)^{\left\lfloor m^{\beta}\right\rfloor}\right)^{\left\lfloor m^{\beta}\right\rfloor+1} \geq 1-\left(\left\lfloor m^{\beta}\right\rfloor+1\right)(1-p)^{\left\lfloor m^{\beta}\right\rfloor}$. Therefore $\mathbb{P}\left(E_{m}^{c}\right) \leq C_{23} m^{\beta} \exp \left(-C_{24} m^{\beta}\right)$ for suitable choice of $C_{23}$ and $C_{24}$.

Fix any $\delta \in(2 \beta, 1)$. Let $l=\min \left\{j: h^{j}(\mathbf{0})(2) \geq\left\lfloor m^{\beta}\right\rfloor+1\right\}$. Note that at every step the path moves a distance at least 1 in the $y$ co-ordinate, hence $l \leq\left\lfloor m^{\beta}\right\rfloor+1$. To complete the proof, it is enough to show that on the set $E_{m}$, we have $\left\{h^{j}(\mathbf{0}): 0 \leq j \leq l\right\} \subseteq\left[-m^{\delta}, m^{\delta}\right] \times\left[0,2\left(\left\lfloor m^{\beta}\right\rfloor+1\right)\right]$.

On the event $E_{m}$, the existence of an open vertex in the first block $\left[1,\left\lfloor m^{\beta}\right\rfloor\right] \times$ $\left\{\left\lfloor m^{\beta}\right\rfloor+1\right\}$ ensures that $\left\|h^{1}(\mathbf{0})\right\|_{1} \leq 2 m^{\beta}+1$. The construction of the set $E_{m}$ ensures that this argument can be repeated for each of the steps until the $l$ th step of the path, i.e. the step when the path crosses $\left\{y=\left\lfloor m^{\beta}\right\rfloor+1\right\}$. Since $l \leq\left\lfloor m^{\beta}\right\rfloor+1$, we have that until the $l$ th step the path stays inside the rectangle $\left[-\left(2\left\lfloor m^{\beta}\right\rfloor+1\right)\left\lfloor m^{\beta}\right\rfloor,\left(2\left\lfloor m^{\beta}\right\rfloor+1\right)\left\lfloor m^{\beta}\right\rfloor\right] \times\left[0,2\left(\left\lfloor m^{\beta}\right\rfloor+1\right)\right]$.

The proposition follows for any $\delta>2 \beta$.

Returning to the verification of condition $\left(I_{1}\right)$ we start with a map $o_{n}: \mathbb{R}^{2} \rightarrow V$ given by

$$
o_{n}(\mathbf{z})=\mathbf{z}_{n}
$$

where $\mathbf{z}_{n}(1):=\min \left\{j+\lfloor n \sigma \mathbf{z}(1)\rfloor: j \geq 0,\left(j+\lfloor n \sigma \mathbf{z}(1)\rfloor,\left\lfloor n^{2} \gamma \mathbf{z}(2)\right\rfloor\right)\right.$ is open $\}$ and $\mathbf{z}_{n}(2):=\left\lfloor n^{2} \gamma \mathbf{z}(2)\right\rfloor$. We now define the path $\zeta_{n}^{\mathbf{z}} \in \mathcal{X}_{n}$ as follows

$$
\zeta_{n}^{\mathbf{z}}:=\pi_{n}^{o_{n}(\mathbf{z})} \text {, for any } \mathbf{z} \in \mathbb{R}^{2} .
$$

Corollary 5.2 .1 proves Condition $\left(I_{1}\right)$ for $k=1$.

We proceed to prove it for $k \geq 2$, assuming that it is true for $k-1$. Fix $\mathbf{x}^{1}, \ldots, \mathbf{x}^{k} \in \mathbb{R}^{2}$, and without loss of generality we assume $\mathbf{x}^{k}(2)=\min \left\{\mathbf{x}^{i}(2): 1 \leq\right.$ $i \leq k\}=0$. 
The strategy we adopt is to show that until the time when the $k$ th path comes close to one of the other $(k-1)$ paths, it can be approximated by an independent path with the same distribution as itself, and after that time, it quickly coalesces with the path which is close to it and both of them converge to the same Brownian motion.

Following the ideas introduced in Ferrari et al. [10], we consider the product metric space $\left(\Pi^{k}, d_{\Pi}^{k}\right)$ where

$$
d_{\Pi}^{k}\left(\left(\pi_{1}, \ldots, \pi_{k}\right),\left(\theta_{1}, \ldots, \theta_{k}\right)\right):=\sum_{i=1}^{k} d_{\Pi}\left(\pi_{i}, \theta_{i}\right) .
$$

We define a subset $A$ of $\Pi^{k}$ as follows:

$A=\left\{\left(\pi_{1}, \ldots, \pi_{k}\right) \in \Pi^{k}:\right.$ such that

a) $\pi_{k}\left(\sigma_{\pi_{j}}\right) \neq \pi_{j}\left(\sigma_{\pi_{j}}\right)$ for all $j \neq k$;

b) $t^{k}:=\inf \left\{t>\max \left\{\sigma_{\pi_{i}}, \sigma_{\pi_{k}}\right\}: \pi_{i}(t)=\pi_{k}(t)\right.$ for some $\left.1 \leq i \leq k-1\right\}<\infty$;

c) for any $\delta>0$ there exist $1 \leq i \leq k-1, t^{k}-\delta<t<t^{k}<s<t^{k}+\delta$ such that $\left(\pi_{k}(t)-\pi_{i}(t)\right)\left(\pi_{k}(s)-\pi_{i}(s)\right)<0$ where $\pi_{i}\left(t^{k}\right)=\pi_{k}\left(t^{k}\right)$ and $\pi_{j}\left(t^{k}\right) \neq \pi_{k}\left(t^{k}\right)$ for all $\left.1 \leq j<i\right\}$.

Note that $A$ consists of all $k$-tuples of continuous paths such that the $k$ th path intersects at least one of the other $k-1$ paths $\pi_{1}, \ldots, \pi_{k-1}$ and it immediately crosses one particular such path. Let $B^{\mathrm{x}^{k}}$ be a standard Brownian motion starting at $\mathbf{x}^{k}$ and independent of $W^{\mathbf{x}^{1}}, \ldots, W^{\mathbf{x}^{k-1}}$, the independent coalescing Brownian motions starting from $\mathbf{x}^{1}, \ldots, \mathbf{x}^{k-1}$. From the path property of independent Brownian motions, we have

$$
\mathbb{P}\left[\left(W^{\mathbf{x}^{1}}, \ldots, W^{\mathbf{x}^{k-1}}, B^{\mathbf{x}^{k}}\right) \in A\right]=1 .
$$

We define a coalescence map $f: \Pi^{k} \rightarrow \Pi^{k}$ as follows:

$$
f\left(\pi_{1}, \ldots, \pi_{k}\right):= \begin{cases}\left(\pi_{1}, \ldots, \pi_{k-1}, \bar{\pi}_{k}\right) & \text { for }\left(\pi_{1}, \ldots, \pi_{k}\right) \in A \\ \left(\pi_{1}, \ldots, \pi_{k}\right) & \text { otherwise }\end{cases}
$$

with

$$
\bar{\pi}_{k}(t):= \begin{cases}\pi_{k}(t) & \text { for } t \leq t^{k} \\ \pi_{i}(t) & \text { for } t>t^{k}\end{cases}
$$


where $i$ is the index such that $\pi_{i}\left(t^{k}\right)=\pi_{k}\left(t^{k}\right)$ and $\pi_{j}\left(t^{k}\right) \neq \pi_{k}\left(t^{k}\right)$ for all $1 \leq j<i$. It follows that

$$
f\left(W^{\mathbf{x}^{1}}, \ldots, W^{\mathbf{x}^{k-1}}, B^{\mathbf{x}^{k}}\right) \stackrel{d}{=}\left(W^{\mathbf{x}^{1}}, \ldots, W^{\mathbf{x}^{k}}\right) .
$$

Next, we define a sequence of subsets of $\Pi^{k}$ where the $k$ th path comes close to one of the $k-1$ paths and a sequence of 'coalescing functions'. The idea of the subsets of $\Pi^{k}$ and coalescing functions is motivated from Ferrari et al. [10. Ferrari et al. considered a sequence of subsets of $\Pi^{k}$ where any two of the $k$ paths come close to each other and defined a sequence of coalescing maps such that before coalescing those two paths are independent. As we proceed by method of induction, we consider subsets of $\Pi^{k}$ where the $k$ th path comes close to one of the $k-1$ paths. Our coalescing map ensures that the probability that before coalescence the $k$ th path is independent of the $k-1$ paths converges to 1 .

We fix $\alpha \in(0,1 / 2)$ for the rest of this section. For $n \geq 1$, define

$$
\begin{gathered}
A_{n}^{\alpha}=\left\{\left(\pi_{1}, \ldots, \pi_{k}\right) \in \Pi^{k}:\right. \text { such that } \\
t_{n}^{k}:=\inf \left\{t \geq \max \left\{\sigma_{\pi_{i}}, \sigma_{\pi_{k}}\right\}:\left|\pi_{i}(t)-\pi_{k}(t)\right| \leq n^{\alpha-1}\right. \\
\quad \text { for some } 1 \leq i \leq k-1\}<\infty\} .
\end{gathered}
$$

We now define the ' $\alpha$-coalescence map' $f_{n}^{(\alpha)}: \Pi^{k} \rightarrow \Pi^{k}$, as follows:

$$
f_{n}^{(\alpha)}\left(\pi_{1}, \ldots, \pi_{k}\right):= \begin{cases}\left(\pi_{1}, \ldots, \pi_{k-1}, \bar{\pi}_{k}\right) & \text { for }\left(\pi_{1}, \ldots, \pi_{k}\right) \in A_{n}^{\alpha} \\ \left(\pi_{1}, \ldots, \pi_{k}\right) & \text { otherwise }\end{cases}
$$

with

$$
\bar{\pi}_{k}(t):= \begin{cases}\pi_{k}(t) & \text { for } t \leq t_{n}^{k} \\ \pi_{k}\left(t_{n}^{k}\right)+\frac{\left(t-t_{n}^{k}\right)}{s_{n}^{k}-t_{n}^{k}}\left[\pi_{i}\left(s_{n}^{k}\right)-\pi_{k}\left(t_{n}^{k}\right)\right] & \text { for } t_{n}^{k}<t \leq s_{n}^{k} \\ \pi_{i}(t) & \text { for } t>s_{n}^{k}\end{cases}
$$

where $s_{n}^{k}=\left(\left\lfloor n^{2} \gamma t_{n}^{k}\right\rfloor+1\right) /\left(n^{2} \gamma\right)$ and $i$ is the index such that $\left|\pi_{i}\left(t_{n}^{k}\right)-\pi_{k}\left(t_{n}^{k}\right)\right| \leq n^{\alpha-1}$ and $\left|\pi_{j}\left(t_{n}^{k}\right)-\pi_{k}\left(t_{n}^{k}\right)\right|>n^{\alpha-1}$ for all $1 \leq j<i$.

Before proceeding, we state the following deterministic lemma (which is a slightly stronger version of Lemma 19 of Coletti et al. [7]). The proof of this lemma has been relegated to the Appendix and it will be used later in the proof of Proposition 5.6 .

Lemma 5.5. Let $\left(\pi_{1}, \ldots, \pi_{k}\right) \in A$ and $\left\{\left(\pi_{1, n}, \ldots, \pi_{k, n}\right): n \geq 1\right\} \subseteq \Pi^{k}$ be such that for all $1 \leq i \leq k, d_{\Pi}\left(\pi_{i, n}, \pi_{i}\right) \rightarrow 0$ as $n \rightarrow \infty$. Then, for $n$ large enough, we have $\left(\pi_{1, n}, \ldots, \pi_{k, n}\right) \in A_{n}^{\alpha}$ and $\lim _{n \rightarrow \infty} t_{n}^{k}=t^{k}$, where $t^{k}, t_{n}^{k}$ are as defined above. Further,

$$
d_{\Pi}^{k}\left(f_{n}^{(\alpha)}\left(\pi_{1, n}, \ldots, \pi_{k, n}\right), f\left(\pi_{1}, \ldots, \pi_{k}\right)\right) \rightarrow 0 \text { as } n \rightarrow \infty .
$$


We now describe a construction which will be used to prove the general case. Let $\left\{U_{\mathbf{w}}^{r}: \mathbf{w} \in \mathbb{Z}^{2}\right\}$ and $\left\{U_{\mathbf{w}}^{g}: \mathbf{w} \in \mathbb{Z}^{2}\right\}$ be two independent collections of i.i.d. $U(0,1)$ random variables. Given a set of points $\mathbf{x}^{1}, \ldots, \mathbf{x}^{k} \in \mathbb{R}^{2}$, let $\left\{\left(\mathbf{x}_{n}^{1}, \ldots, \mathbf{x}_{n}^{k}\right)\right.$ : $n \geq 1\}$ be such that for all $i=1,2, \ldots, k, \mathbf{x}_{n}^{i} \in \mathbb{Z}^{2}$ for $n \geq 1$ with $\mathbf{x}_{n}^{i}(2) \geq 0$ and $\left(\mathbf{x}_{n}^{i}(1) / n \sigma, \mathbf{x}_{n}^{i}(2) / n^{2} \gamma\right) \rightarrow \mathbf{x}^{i}$ as $n \rightarrow \infty$. We construct the paths $\pi^{1}, \ldots, \pi^{k-1}$ starting from $\mathbf{x}_{n}^{1}, \ldots, \mathbf{x}_{n}^{k-1}$ using only the collection $\left\{U_{\mathbf{w}}^{r}: \mathbf{w} \in \mathbb{Z}^{2}\right\}$, while, for the construction of the path $\tilde{\pi}^{k}$ starting from $\mathbf{x}_{n}^{k}$ we use the collection $\left\{U_{\mathbf{w}}^{g}: \mathbf{w} \in \mathbb{Z}^{2}\right\}$. From the independence of the collections of uniform random variables, the scaled paths $\left(\pi_{n}^{1}, \ldots, \pi_{n}^{k-1}\right)$ and the scaled path $\tilde{\pi}_{n}^{k}$ are independent. Further,

$$
\left(\pi_{n}^{1}, \ldots, \pi_{n}^{k-1}\right) \stackrel{d}{=}\left(\pi_{n}^{\mathbf{x}_{n}^{1}}, \ldots, \pi_{n}^{\mathbf{x}_{n}^{k-1}}\right) \quad \text { and } \quad \tilde{\pi}_{n}^{k} \stackrel{d}{=} \pi_{n}^{\mathbf{x}_{n}^{k}} .
$$

Next, we consider the region $E^{(r)}$ which is explored by the paths $\pi^{1}, \ldots, \pi^{k-1}$, constructed using the collection $\left\{U_{\mathbf{w}}^{r}: \mathbf{w} \in \mathbb{Z}^{2}\right\}$ only. On the complement set of $E^{(r)}$, we consider the collection $\left\{U_{\mathbf{w}}^{g}: \mathbf{w} \in \mathbb{Z}^{2}\right\}$ and construct the path, $\pi^{k}$, starting from $\mathbf{x}_{n}^{k}$. More precisely, the set

$$
E^{(r)}:=\bigcup_{1 \leq i \leq k-1} \bigcup_{m \geq 0} S^{+}\left(h^{m}\left(\mathbf{x}_{n}^{i}\right),\left\|h^{m}\left(\mathbf{x}_{n}^{i}\right)-h^{m+1}\left(\mathbf{x}_{n}^{i}\right)\right\|_{1}\right)
$$

represents the explored region by the paths $\pi^{1}, \ldots, \pi^{k-1}$ using the collection $\left\{U_{\mathbf{w}}^{r}\right.$ : $\left.\mathbf{w} \in \mathbb{Z}^{2}\right\}$ only. We define $\left\{U_{\mathbf{w}}^{\text {mixed }}: \mathbf{w} \in \mathbb{Z}^{2}\right\}$ by

$$
U_{\mathbf{w}}^{\text {mixed }}:= \begin{cases}U_{\mathbf{w}}^{r} & \text { if } \mathbf{w} \in E^{(r)} \\ U_{\mathbf{w}}^{g} & \text { otherwise. }\end{cases}
$$

Let $\pi^{k}$ be the path starting from $\mathbf{x}_{n}^{k}$ constructed using the collection $\left\{U_{\mathbf{w}}^{\text {mixed }}\right.$ : $\left.\mathbf{w} \in \mathbb{Z}^{2}\right\}$. We also observe that the distribution of $\pi^{k}$, given the realization of the uniform random variables in $E^{(r)}$, is the same as the conditional distribution of $\pi^{\mathbf{x}_{n}^{k}}$ given the paths $\pi^{\mathbf{x}_{n}^{1}}, \ldots, \pi^{\mathbf{x}_{n}^{k-1}}$. Hence from the above observation and definition (31),

$$
\left(\pi_{n}^{1}, \ldots, \pi_{n}^{k-1}, \pi_{n}^{k}\right) \stackrel{d}{=}\left(\pi_{n}^{\mathbf{x}_{n}^{1}}, \ldots, \pi_{n}^{\mathbf{x}_{n}^{k-1}}, \pi_{n}^{\mathbf{x}_{n}^{k}}\right) .
$$

Proposition 5.6. We have, as $n \rightarrow \infty$,

$$
\begin{aligned}
& \text { (a) } f_{n}^{(\alpha)}\left(\pi_{n}^{1}, \ldots, \pi_{n}^{k-1}, \tilde{\pi}_{n}^{k}\right) \Rightarrow\left(W^{\mathbf{x}^{1}}, \ldots, W^{\mathbf{x}^{k}}\right) ; \\
& \text { (b) } f_{n}^{(\alpha)}\left(\pi_{n}^{1}, \ldots, \pi_{n}^{k-1}, \pi_{n}^{k}\right) \Rightarrow\left(W^{\mathbf{x}^{1}}, \ldots, W^{\mathbf{x}^{k}}\right) \\
& \text { (c) }\left(\pi_{n}^{1}, \ldots, \pi_{n}^{k-1}, \pi_{n}^{k}\right) \Rightarrow\left(W^{\mathbf{x}^{1}}, \ldots, W^{\mathbf{x}^{k}}\right) .
\end{aligned}
$$

Since $\left(o_{n}(\mathbf{z})(1) /(n \sigma), o_{n}(\mathbf{z})(2) /\left(n^{2} \gamma\right)\right) \rightarrow \mathbf{z}$ almost surely, where $o_{n}(\mathbf{z})$ is defined in (30), by (c) of the proposition above, we have

$$
\left(\zeta_{n}^{\mathbf{x}^{1}}, \ldots, \zeta_{n}^{\mathbf{x}^{k}}\right) \Rightarrow\left(W^{\mathbf{x}^{1}}, \ldots, W^{\mathbf{x}^{k}}\right),
$$


which verifies $\left(I_{1}\right)$.

Proof: From Corollary 5.2.1 and (36) it follows that the scaled path $\tilde{\pi}_{n}^{k}$ converges in distribution to $B^{\mathbf{x}^{k}}$, the standard Brownian motion starting at $\mathbf{x}^{k}$. Using the induction hypothesis and (36) we have that $\left(\pi_{n}^{1}, \ldots, \pi_{n}^{k-1}\right)$ converges in distribution to $\left(W^{\mathbf{x}^{1}}, \ldots, W^{\mathbf{x}^{k-1}}\right)$. From the independence of paths we have $\left(\pi_{n}^{1}, \ldots, \pi_{n}^{k-1}, \tilde{\pi}_{n}^{k}\right)$ converges in distribution to $\left(W^{\mathbf{x}^{1}}, \ldots, W^{\mathbf{x}^{k-1}}, B^{\mathbf{x}^{k}}\right)$ where $B^{\mathbf{x}^{k}}$ is independent of $\left(W^{\mathbf{x}^{1}}, \ldots, W^{\mathbf{x}^{k-1}}\right)$. Now Lemma 5.5 and (32) enable us to use the extended continuous mapping theorem (see Theorem 4.27 in Kallenberg [15]) to conclude that

$$
f_{n}^{(\alpha)}\left(\pi_{n}^{1}, \ldots, \pi_{n}^{k-1}, \tilde{\pi}_{n}^{k}\right) \Rightarrow f\left(W^{\mathbf{x}^{1}}, \ldots, W^{\mathbf{x}^{k-1}}, B^{\mathbf{x}^{k}}\right) \stackrel{d}{=}\left(W^{\mathbf{x}^{1}}, \ldots, W^{\mathbf{x}^{k}}\right)
$$

where the last relation follows from (33). This proves (a).

For $(b)$, let $f_{n}^{(\alpha)}\left(\pi_{n}^{1}, \ldots, \pi_{n}^{k-1}, \pi_{n}^{k}\right)=\left(\pi_{n}^{1}, \ldots, \pi_{n}^{k-1}, \bar{\pi}_{n}^{k}\right)$ and $f_{n}^{(\alpha)}\left(\pi_{n}^{1}, \ldots, \pi_{n}^{k-1}\right.$, $\left.\tilde{\pi}_{n}^{k}\right)=\left(\pi_{n}^{1}, \ldots, \pi_{n}^{k-1}, \overline{\bar{\pi}}_{n}^{k}\right)$. Note that $d_{\Pi}^{k}\left(\left(\pi_{n}^{1}, \ldots, \pi_{n}^{k-1}, \bar{\pi}_{n}^{k}\right),\left(\pi_{n}^{1}, \ldots, \pi_{n}^{k-1}, \overline{\bar{\pi}}_{n}^{k}\right)\right)=$ $d_{\Pi}\left(\bar{\pi}_{n}^{k}, \overline{\bar{\pi}}_{n}^{k}\right)$. Hence it is enough to show that $d_{\Pi}\left(\bar{\pi}_{n}^{k}, \overline{\bar{\pi}}_{n}^{k}\right) \stackrel{\mathbb{P}}{\rightarrow} 0$ as $n \rightarrow \infty$. Since both the paths start at the same point, it is enough to prove that,

$$
\text { for any } t>0, \sup \left\{\left|\bar{\pi}_{n}^{k}(s)-\overline{\bar{\pi}}_{n}^{k}(s)\right|: 0 \leq s \leq t\right\} \stackrel{\mathbb{P}}{\rightarrow} 0 \text { as } n \rightarrow \infty \text {. }
$$

Towards this end, we show that on a set whose probability converges to $1, \bar{\pi}_{n}^{k}(s)$ and $\overline{\bar{\pi}}_{n}^{k}(s)$ agree for $s \in[0, t]$.

For any $s>0$ and $i=1,2, \ldots, k$, set

$$
l_{i}^{(s)}=l_{i}^{(s)}(n):=\min \left\{j \geq 0: T_{j}\left(\mathbf{x}_{n}^{i}\right) \geq n^{2} \gamma s\right\}
$$

where $T_{j}(\mathbf{u})$ is the $j$ th regeneration time for path starting from $\mathbf{u}$ defined in (10). Here $l_{i}^{(s)}$ stands for the number of regenerations needed for the $i$ th path $\pi^{i}$ (starting from $\mathbf{x}_{n}^{i}$ ) to cross the line $\left\{y=n^{2} \gamma s\right\}$. Now, for $0<\beta<\alpha$ and any $s>0$, define the event

$$
A_{n}^{(s)}:=\left\{W_{j}\left(\mathbf{x}_{n}^{i}\right)<n^{\beta}: \text { for all } 1 \leq i \leq k, 1 \leq j \leq l_{i}^{(s)}\right\} .
$$

with $W_{j}(\mathbf{u})$ as defined in (9)). On the event $A_{n}^{(s)}$, each of the regeneration steps till the $l_{i}^{(s)}$ th regeneration of all the $k$ paths, is of length at most $n^{\beta}$. Since $l_{i}^{(s)} \leq\left\lfloor n^{2} \gamma s\right\rfloor+1$, and using the fact that the individual regenerations are i.i.d., having bounds on tail probabilities given in Proposition 2.8, we have, as $n \rightarrow \infty$,

$$
\begin{aligned}
\mathbb{P}\left(\left(A_{n}^{(s)}\right)^{c}\right) & =\mathbb{P}\left(\bigcup_{1 \leq i \leq k} \bigcup_{1 \leq j \leq l_{i}^{(s)}}\left\{W_{j}\left(\mathbf{x}_{n}^{i}\right) \geq n^{\beta}\right\}\right) \\
& \leq k\left(\left\lfloor n^{2} \gamma s\right\rfloor+1\right) \mathbb{P}\left(W_{1}(\mathbf{0}) \geq n^{\beta}\right) \rightarrow 0 .
\end{aligned}
$$

If $t_{n}^{k} \geq t$, on the event $A_{n}^{(t)}$, 
(a) from the definition of $t_{n}^{k}$, for $s \leq n^{2} \gamma t$, we have $\min _{1 \leq i \leq k-1}\left|\tilde{\pi}^{k}(s)-\pi^{i}(s)\right| \geq$ $n^{\alpha}$

(b) the restriction on the sizes of the regeneration steps by $n^{\beta}$ together with the choice $\beta<\alpha$ ensures that the path $\tilde{\pi}^{k}$ has not explored the region $E^{(r)}$ before it crosses $\left\{y=n^{2} \gamma t\right\}$.

Thus the paths $\tilde{\pi}^{k}$ and $\pi^{k}$ agree on $\left[0, n^{2} \gamma t\right]$ and so $\bar{\pi}_{n}^{k}(s)=\overline{\bar{\pi}}_{n}^{k}(s)$ for $0 \leq s \leq t$.

If $t_{n}^{k} \leq t$, on the event $A_{n}^{\left(t_{n}^{k}\right)}$, we have

(a) from the definition of $t_{n}^{k}$, for every $s \leq n^{2} \gamma t_{n}^{k}$, we have $\min _{1 \leq i \leq k-1} \mid \tilde{\pi}^{k}(s)-$ $\pi^{i}(s) \mid \geq n^{\alpha}$,

(b) the restriction on the sizes of the regeneration steps by $n^{\beta}$ together with the choice $\beta<\alpha$ ensures that the path $\tilde{\pi}^{k}$ has not explored the region $E^{(r)}$ before it crosses $\left\{y=n^{2} \gamma t_{n}^{k}\right\}$.

Thus the paths $\tilde{\pi}^{k}$ and $\pi^{k}$ agree on $\left[0, n^{2} \gamma t_{n}^{k}\right]$ and thereby $\bar{\pi}^{k}(s)=\overline{\bar{\pi}}^{k}(s)$ for $0 \leq$ $s \leq t_{n}^{k}$. The rest of the path $\pi^{k}$ (from $n^{2} \gamma t_{n}^{k}$ onwards) depends only on the position $\tilde{\pi}^{k}\left(n^{2} \gamma t_{n}^{k}\right)$ and the paths $\left(\pi^{1}, \ldots, \pi^{k-1}\right)$ and hence, by the definition of the $\alpha$-coalescing map, we have

$$
f_{n}^{(\alpha)}\left(\pi_{n}^{1}, \ldots, \pi_{n}^{k-1}, \pi_{n}^{k}\right)(s)=f_{n}^{(\alpha)}\left(\pi_{n}^{1}, \ldots, \pi_{n}^{k-1}, \tilde{\pi}_{n}^{k}\right)(s)
$$

for $s \in\left[t_{n}^{k}, t\right]$. Hence, $\bar{\pi}_{n}^{k}(s)=\overline{\bar{\pi}}_{n}^{k}(s)$ for $0 \leq s \leq t$.

Since $A_{n}^{(t)} \subseteq A_{n}^{\left(t_{n}^{k}\right)}$ when $t_{n}^{k} \leq t$, (39) along with the two observations above implies (38). This completes the proof of part (b).

For $(c)$, we show that $d_{\Pi}^{k}\left(\left(\pi_{n}^{1}, \ldots, \pi_{n}^{k-1}, \bar{\pi}_{n}^{k}\right),\left(\pi_{n}^{1}, \ldots, \pi_{n}^{k-1}, \pi_{n}^{k}\right)\right)=d_{\Pi}\left(\bar{\pi}_{n}^{k}, \pi_{n}^{k}\right) \stackrel{\mathbb{P}}{\rightarrow}$ 0 as $n \rightarrow \infty$. Again it is enough to prove,

$$
\text { for any } t>0, \sup \left\{\left|\pi_{n}^{k}(s)-\bar{\pi}_{n}^{k}(s)\right|: 0 \leq s \leq t\right\} \stackrel{\mathbb{P}}{\rightarrow} 0 \text { as } n \rightarrow \infty \text {. }
$$

Suppose $i_{0}:=\min \left\{j:\left|\pi_{n}^{k}\left(t_{n}^{k}\right)-\pi_{n}^{j}\left(t_{n}^{k}\right)\right| \leq n^{\alpha-1}\right\}$, i.e. $\pi_{n}^{i_{0}}$ is the path with the minimum index which comes $n^{\alpha-1}$ close to $\pi_{n}^{k}$. Note that $\bar{\pi}_{n}^{k}(s)$ is obtained by a linear interpolation between $\pi_{n}^{k}\left(t_{n}^{k}\right)$ and $\pi_{n}^{i_{0}}\left(s_{n}^{k}\right)$ for $s \in\left[t_{n}^{k}, s_{n}^{k}\right]$ and $\bar{\pi}_{n}^{k}(s)=\pi_{n}^{i_{0}}(s)$ for $s \in\left[s_{n}^{k}, \infty\right)$ where $s_{n}^{k}=\left(\left\lfloor n^{2} \gamma t_{n}^{k}\right\rfloor+1\right) /\left(n^{2} \gamma\right)$. Since paths in $\mathcal{X}_{n}$ are noncrossing almost surely, we have $\bar{\pi}_{n}^{k}(s) \in\left[\pi_{n}^{i_{0}}(s), \pi_{n}^{k}(s)\right]$ for $\left[t_{n}^{k}, \infty\right)$. Also, note that both the paths $\pi_{n}^{k}$ and $\bar{\pi}_{n}^{k}$ start at the same point and agree till $t_{n}^{k}$. Thus (40) holds for $t_{n}^{k} \geq t$

If $t_{n}^{k}<t$, we have

$$
\sup \left\{\left|\pi_{n}^{k}(s)-\bar{\pi}_{n}^{k}(s)\right|: 0 \leq s \leq t\right\} \leq \sup \left\{\left|\pi_{n}^{k}(s)-\pi_{n}^{i_{0}}(s)\right|: t_{n}^{k} \leq s \leq t\right\} .
$$


Again, we restrict ourselves to the event $A_{n}^{\left(t_{n}^{k}\right)}$. Let $\Delta_{n}$ be the set of vertices explored by the paths until all of them have crossed the line $\left\{y=\left\lfloor n^{2} \gamma t_{n}^{k}\right\rfloor\right\}$, i.e.,

$$
\Delta_{n}=\bigcup_{1 \leq i \leq k} \bigcup_{m=0}^{\substack{\tau_{i}^{\left(t_{n}^{k}\right)}-1 \\ l^{\prime}}} S^{+}\left(h^{m}\left(\mathbf{x}_{n}^{i}\right),\left\|h^{m}\left(\mathbf{x}_{n}^{i}\right)-h^{m+1}\left(\mathbf{x}_{n}^{i}\right)\right\|_{1}\right)
$$

We observe that, on the event $A_{n}^{\left(t_{n}^{k}\right)}$, the set $\Delta_{n}$ is contained in $\mathbb{H}\left(\left\lfloor n^{2} \gamma t_{n}^{k}\right\rfloor+\left\lfloor n^{\beta}\right\rfloor\right)$. Now we choose two points $u_{n}, v_{n} \in \mathbb{Z}$ such that $u_{n}<\pi^{i_{0}}\left(\left\lfloor n^{2} \gamma t_{n}^{k}\right\rfloor\right), \pi^{k}\left(\left\lfloor n^{2} \gamma t_{n}^{k}\right\rfloor\right)<$ $v_{n}$ and $\left(v_{n}-u_{n}\right) / n \rightarrow 0$. Since $\left|\pi^{i_{0}}\left(\left\lfloor n^{2} \gamma t_{n}^{k}\right\rfloor\right)-\pi^{k}\left(\left\lfloor n^{2} \gamma t_{n}^{k}\right\rfloor\right)\right| \leq n^{\alpha-1}$, such a choice of $u_{n}, v_{n}$ is possible for $n$ large. Set $\mathbf{u}_{n}:=\left(u_{n},\left\lfloor n^{2} \gamma t_{n}^{k}\right\rfloor\right)$ and $\mathbf{v}_{n}:=\left(v_{n},\left\lfloor n^{2} \gamma t_{n}^{k}\right\rfloor\right)$. We consider scaled paths $\pi_{n}^{\mathbf{u}_{n}}$ and $\pi_{n}^{\mathbf{v}_{n}}$ and by noncrossing property of the paths, we see that the paths $\pi_{n}^{i_{0}}$ and $\pi_{n}^{k}$ lie between the paths $\pi_{n}^{\mathbf{u}_{n}}$ and $\pi_{n}^{\mathbf{v}_{n}}$ from $t_{n}^{k}$ onwards, so that

$$
\sup \left\{\left|\pi_{n}^{k}(s)-\pi_{n}^{i_{0}}(s)\right|: t_{n}^{k} \leq s \leq t\right\} \leq \sup \left\{\left|\pi_{n}^{\mathbf{v}_{n}}(s)-\pi_{n}^{\mathbf{u}_{n}}(s)\right|: t_{n}^{k} \leq s \leq t\right\} .
$$

Fix any $\delta \in(2 \beta, 1)$ and consider the points $\mathbf{u}_{n}^{\prime}=\left(u_{n}-\left\lfloor n^{\delta}\right\rfloor-1,\left\lfloor n^{2} \gamma t_{n}^{k}\right\rfloor+\right.$ $\left.\left\lfloor n^{\beta}\right\rfloor+1\right)$ and $\mathbf{v}_{n}^{\prime}=\left(v_{n}+\left\lfloor n^{\delta}\right\rfloor+1,\left\lfloor n^{2} \gamma t_{n}^{k}\right\rfloor+\left\lfloor n^{\beta}\right\rfloor+1\right)$. Let

$$
\begin{aligned}
& F_{n}\left(\mathbf{u}_{n}\right):=\left\{\sup \left\{\left|\pi^{\mathbf{u}_{n}}(s)-u_{n}\right|:\left\lfloor n^{2} \gamma t_{n}^{k}\right\rfloor \leq s \leq\left\lfloor n^{2} \gamma t_{n}^{k}\right\rfloor+n^{\beta}\right\} \leq n^{\delta}\right\}, \\
& F_{n}\left(\mathbf{v}_{n}\right):=\left\{\sup \left\{\left|\pi^{\mathbf{v}_{n}}(s)-v_{n}\right|:\left\lfloor n^{2} \gamma t_{n}^{k}\right\rfloor \leq s \leq\left\lfloor n^{2} \gamma t_{n}^{k}\right\rfloor+n^{\beta}\right\} \leq n^{\delta}\right\} .
\end{aligned}
$$

By Proposition 5.4 (taking $\mathcal{R}=\Delta_{n}$ and translating so that $\mathbf{u}_{n}$ is the origin) we have $\mathbb{P}\left(F_{n}\left(\mathbf{u}_{n}\right)\right) \rightarrow 1$. Similarly, $\mathbb{P}\left(F_{n}\left(\mathbf{v}_{n}\right)\right) \rightarrow 1$.

On the set $F_{n}\left(\mathbf{u}_{n}\right) \cap F_{n}\left(\mathbf{v}_{n}\right)$, we have that $\mathbf{u}_{n}^{\prime}(1) \leq \pi^{\mathbf{u}_{n}}\left(\left\lfloor n^{2} \gamma t_{n}^{k}\right\rfloor+\left\lfloor n^{\beta}\right\rfloor+\right.$ 1), $\pi^{\mathbf{v}_{n}}\left(\left\lfloor n^{2} \gamma t_{n}^{k}\right\rfloor+\left\lfloor n^{\beta}\right\rfloor+1\right) \leq \mathbf{v}_{n}^{\prime}(1)$ and hence, by non-crossing property of paths, $\pi^{\mathbf{u}_{n}^{\prime}}(s) \leq \pi^{\mathbf{u}_{n}}(s) \leq \pi^{\mathbf{v}_{n}}(s) \leq \pi^{\mathbf{v}_{n}^{\prime}}(s)$ for all $s>\left\lfloor n^{2} \gamma t_{n}^{k}\right\rfloor+\left\lfloor n^{\beta}\right\rfloor+1$. Using the estimate in Proposition 5.4, we have

$$
\begin{aligned}
& \sup \left\{\left|\pi_{n}^{\mathbf{u}_{n}}(s)-\pi_{n}^{\mathbf{v}_{n}}(s)\right|: t_{n}^{k} \leq s \leq t\right\} \\
& \leq\left(\left|u_{n}-v_{n}\right|+2 n^{\delta}\right) /(n \sigma)+\sup \left\{\left|\pi_{n}^{\mathbf{u}_{n}^{\prime}}(s)-\pi_{n}^{\mathbf{v}_{n}^{\prime}}(s)\right|: t_{n}^{k}+n^{\beta} /\left(n^{2} \gamma\right) \leq s \leq t\right\} .
\end{aligned}
$$

The paths starting from $\mathbf{u}_{n}^{\prime}$ and $\mathbf{v}_{n}^{\prime}$ depend only on the uniform random variables defined on $\left\{y>\left\lfloor n^{2} \gamma t_{n}^{k}\right\rfloor+\left\lfloor n^{\beta}\right\rfloor+1\right\}$ and hence independent of the realizations in the set $\Delta_{n}$. Using translation invariance of our model and Proposition 5.3 we conclude that

$$
\sup \left\{\left|\pi_{n}^{\mathbf{u}_{n}^{\prime}}(s)-\pi_{n}^{\mathbf{v}_{n}^{\prime}}(s)\right|: t_{n}^{k}+n^{\beta} /\left(n^{2} \gamma\right) \leq s \leq t\right\} \stackrel{\mathbb{P}}{\rightarrow} 0
$$

This proves the proposition. 


\subsection{Verification of $\left(B_{1}\right)$ and $\left(E_{1}^{\prime}\right)$}

The verification of condition $\left(B_{1}\right)$ is standard and follows from the same argument as in Ferrari et al. [10]. Recall that for $t_{0} \in \mathbb{R}, t>0$ and $-\infty<a<b<\infty$ and for $\Gamma \subseteq \Pi$

$$
\eta_{\Gamma}\left(t_{0}, t ; a, b\right)=\#\left\{\pi\left(t_{0}+t\right): \pi \in \Gamma^{t_{0}} \text { and } \pi\left(t_{0}\right) \in[a, b]\right\} .
$$

By translation invariance, it suffices to consider $\eta_{\overline{\mathcal{X}}_{n}}(0, t ; 0, \epsilon)$. Let $\mathbf{v}^{n}=((\lfloor n \sigma \epsilon\rfloor+$ $1), 0)$. As noted in [10], using $\left(I_{1}\right)$, we have $\mathbb{P}\left(\eta_{\overline{\mathcal{X}}_{n}}(0, t ; 0, \epsilon) \geq 2\right) \leq \mathbb{P}\left(\pi_{n}^{\mathbf{0}}(t) \neq\right.$ $\left.\pi_{n}^{\mathbf{v}^{n}}(t)\right) \rightarrow \mathbb{P}\left(W^{\mathbf{0}}(t) \neq W^{(\epsilon, 0)}(t)\right)=2 \phi(\epsilon / \sqrt{t})-1$.

Hence we have

$$
\limsup _{n \rightarrow \infty} \mathbb{P}\left(\eta_{\overline{\mathcal{X}}_{n}}(0, t ; 0, \epsilon) \geq 2\right) \leq 2 \Phi\left(\frac{\epsilon}{\sqrt{t}}\right)-1 \rightarrow 0
$$

as $\epsilon \rightarrow 0$ which verifies $\left(B_{1}\right)$.

In order to verify $\left(E_{1}^{\prime}\right)$, recall that

$$
\overline{\mathcal{X}}_{n}^{t_{0}}=\left\{\pi: \pi \in \overline{\mathcal{X}}_{n} \text { with } \sigma_{\pi} \leq t_{0}\right\}
$$

Since $\overline{\mathcal{X}}_{n}^{t_{0}} \subseteq \overline{\mathcal{X}}_{n}$ for any $t_{0} \in \mathbb{R}$, and $\left\{\overline{\mathcal{X}}_{n}: n \geq 1\right\}$ is tight, we have $\left\{\overline{\mathcal{X}}_{n}^{t_{0}}: n \geq 1\right\}$ is also tight. Let $\mathcal{Z}^{t_{0}}$ be a subsequential limit of $\left\{\overline{\mathcal{X}}_{n}^{t_{0}}: n \geq 1\right\}$. For ease of notation, we assume that $\left\{\overline{\mathcal{X}}_{n}^{t_{0}}: n \geq 1\right\}$ is itself the subsequence which converges to $\mathcal{Z}^{t_{0}}$.

For $\Gamma \subseteq \Pi$, let $\Gamma(s):=\left\{(\pi(s), s): \pi \in \Gamma^{s}\right\} \subseteq \mathbb{R}^{2}$. For $t>0$ define $\Gamma^{s ;(s+t)_{T}}:=$ $\left\{\pi: \sigma_{\pi}=s+t\right.$ and there exists $\pi^{\prime} \in \Gamma^{s}$ such that $\pi(u)=\pi^{\prime}(u)$ for all $\left.u \geq s+t\right\}$. Note $\Gamma^{s ;(s+t)_{T}}$ is the restriction of paths in $\Gamma^{s}$ on $[s+t, \infty)$. Following the argument in Newman et al. [17], our strategy to check $\left(E_{1}^{\prime}\right)$ is to first show that the point set $\mathcal{Z}^{t_{0}}\left(t_{0}+t\right)$ is locally finite and then using $\left(I_{1}\right)$ we show that $\left(\mathcal{Z}^{t_{0}}\right)^{t_{0} ;\left(t_{0}+t\right)_{T}}=$ $\mathcal{Z}^{t_{0} ;\left(t_{0}+t\right)_{T}}$ has the same distribution as coalescing Brownian motions $\mathcal{W}^{\mathcal{Z}^{t_{0}}\left(t_{0}+t\right)}$ starting from a random point set distributed as $\mathcal{Z}^{t_{0}}\left(t_{0}+t\right)$.

To show $\mathcal{Z}^{t_{0}}\left(t_{0}+t\right)$ is locally finite, we need to control the tail of the distribution of the coalescing time of two paths starting at the same instant of time.

Proposition 5.7. For $\mathbf{u}, \mathbf{v} \in \mathbb{Z}^{2}, \mathbf{u}(2)=\mathbf{v}(2)$ consider the process, $\left\{Z_{j}(\mathbf{u}, \mathbf{v})\right.$ : $j \geq 0\}$. We have

$$
\sup \left\{\mathbb{P}\left(Z_{j+1}(\mathbf{u}, \mathbf{v})=m \mid Z_{j}(\mathbf{u}, \mathbf{v})=m\right): m \geq 1\right\} \leq \theta
$$

for some $\theta \in(0,1)$ which is independent of $j$.

Proof : To prove Proposition 5.7, we observe that for $m \geq 3, \mathbb{P}\left(Z_{j+1}(\mathbf{u}, \mathbf{v})=\right.$ $\left.m+1 \mid Z_{j}(\mathbf{u}, \mathbf{v})=m\right) \geq(1-p)^{6} p^{3}, \mathbb{P}\left(Z_{j+1}(\mathbf{u}, \mathbf{v})=3 \mid Z_{j}(\mathbf{u}, \mathbf{v})=2\right) \geq(1-p)^{5} p^{3}$ and 
$\mathbb{P}\left(Z_{j+1}(\mathbf{u}, \mathbf{v})=2 \mid Z_{j}(\mathbf{u}, \mathbf{v})=1\right) \geq(1-p)^{4} p^{3}$ (see Figure 7$)$. Therefore, we have

$$
\begin{aligned}
& \mathbb{P}\left(Z_{j+1}(\mathbf{u}, \mathbf{v})=m \mid Z_{j}(\mathbf{u}, \mathbf{v})=m\right) \\
& \leq 1-\mathbb{P}\left(Z_{1}(\mathbf{u}, \mathbf{v})=m+1 \mid Z_{0}(\mathbf{u}, \mathbf{v})=m\right) \\
& \leq 1-\min \left\{(1-p)^{6} p^{3},(1-p)^{5} p^{3},(1-p)^{4} p^{3}\right\}=1-(1-p)^{6} p^{3} .
\end{aligned}
$$

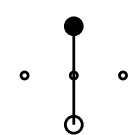

$(0,0)$

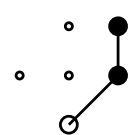

$(m, 0)$

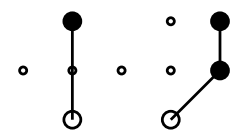

$(0,0)$

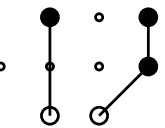

$(0,0) \quad(m, 0)$

Case : $m \geq 3$

Case : $m=2$

Case : $m=1$

Figure 7: One possible realization of the event $\left\{Z_{j+1}=m+1 \mid Z_{j}=m\right\}$. The bold vertices are open and all other vertices depicted are closed.

Now, we prove an estimate on the tail of coalescing time. We will use the following result (Theorem 4 of Coletti et al. [7])

Theorem 5.8. Let $\left\{V_{j}: j \geq 0\right\}$ be a Markov chain on the state space $\{0,1, \ldots\}$ with 0 being the only absorbing state. Further, assume that $\left\{V_{j}: j \geq 0\right\}$ is a martingale and $\sup \left\{\mathbb{P}\left(V_{1}=m \mid V_{0}=m\right): m \geq 1\right\} \leq \theta$ for some constant $\theta \in(0,1)$. Let $\tau^{V}:=\inf \left\{j \geq 1: V_{j}=0\right\}$. Then, for some constant $C_{25}$, we have

$$
\mathbb{P}\left(\tau^{V} \geq n \mid V_{0}=1\right) \leq C_{25} / \sqrt{n} \text { for all } n \geq 1 \text {. }
$$

Proposition 5.9. Fix $\mathbf{u}=(1,0), \mathbf{v}=(0,0) \in \mathbb{Z}^{2}$, let $\nu=\inf \left\{l: g_{\tau_{l}(\mathbf{u}, \mathbf{v})}(\mathbf{u})=\right.$ $\left.g_{\tau_{l}(\mathbf{u}, \mathbf{v})}(\mathbf{v})\right\}$, where $\tau_{l}(\mathbf{u}, \mathbf{v})$ is the $l$ th regeneration step as defined in (1). For the $\nu$ th regeneration time $T_{\nu}(\mathbf{u}, \mathbf{v})$ as defined in (10), there exist positive constants $C_{26}$ and $C_{27}$, such that, we have

$$
\mathbb{P}(\nu \geq t) \leq C_{26} / \sqrt{t} \quad \text { and } \quad \mathbb{P}\left(T_{\nu}(\mathbf{u}, \mathbf{v}) \geq t\right) \leq C_{27} / \sqrt{t}
$$

Proof : The process $\left\{Z_{j}(\mathbf{u}, \mathbf{v}): j \geq 0\right\}$ satisfies the conditions of Theorem 5.8 and therefore, it follows that $\mathbb{P}(\nu \geq t) \leq C_{26} / \sqrt{t}$ where $C_{26}$ is a constant.

To achieve the bound on $T_{\nu}(\mathbf{u}, \mathbf{v})$, we choose $C_{28}=1 /\left(2 \mathbb{E}\left(W^{M}\right)\right)$ where $W^{M}$ is as in Proposition 2.8. Note that, it is also the case that $T_{l}(\mathbf{u}, \mathbf{v}) \leq \sum_{i=1}^{l} W^{M}(i)$, for any $l \geq 1$, where $\left\{W^{M}(i): i \geq 1\right\}$ is an i.i.d. sequence, each having the same distribution as that of $W^{M}$ (see discussion before (10) and the statement of 
Proposition 2.8). We have,

$$
\begin{aligned}
& \mathbb{P}\left(T_{\nu}(\mathbf{u}, \mathbf{v}) \geq t\right) \leq \mathbb{P}\left(T_{\nu}(\mathbf{u}, \mathbf{v}) \geq t, \nu<C_{28} t\right)+\mathbb{P}\left(\nu \geq C_{28} t\right) \\
& \leq \mathbb{P}\left(T_{\left\lfloor C_{28} t\right\rfloor}(\mathbf{u}, \mathbf{v}) \geq t\right)+\frac{C_{26}}{\sqrt{C_{28} t}} \\
& \leq \mathbb{P}\left[\sum_{i=1}^{\left\lfloor C_{28} t\right\rfloor}\left(W^{M}(i)-\mathbb{E}\left(W^{M}(i)\right)\right) \geq t-\mathbb{E}\left(W^{M}\right)\left\lfloor C_{28} t\right\rfloor\right]+\frac{C_{26}}{\sqrt{C_{28} t}} \\
& \leq \frac{\operatorname{Var}\left(\sum_{i=1}^{\left\lfloor C_{28} t\right\rfloor} W^{M}(i)\right)}{\left[t-\mathbb{E}\left(W^{M}\right)\left\lfloor C_{28} t\right\rfloor\right]^{2}}+\frac{C_{26}}{\sqrt{C_{28} t}} \\
& \leq \frac{\left\lfloor C_{28} t\right\rfloor \mathbb{V a r}\left(W^{M}\right)}{(t / 2-1)^{2}}+\frac{C_{26}}{\sqrt{C_{28} t}} \\
& \leq \frac{C_{27}}{\sqrt{t}}
\end{aligned}
$$

for a suitable choice of constant $C_{27}$. This completes the proof.

Before we proceed further, we introduce the following notation: for any $A \subseteq \mathbb{Z}^{2}$, let $\mathcal{X}^{A}$ and $\mathcal{X}_{n}^{A}$ be the collection of paths starting at the vertices of $A$ and their scaled versions respectively, i.e., $\mathcal{X}^{A}:=\left\{\pi^{\mathbf{u}}: \mathbf{u} \in A\right\}$ and $\mathcal{X}_{n}^{A}:=\left\{\pi_{n}^{\mathbf{u}}: \mathbf{u} \in A\right\}$. Now, we prove the following proposition which is an adaptation of Lemma 2.7 of Newman et al. [17].

Proposition 5.10. For $a, b \in \mathbb{R}$ and $t>0$, for all $n \geq 1$, we have

$$
\mathbb{E}\left[\#\left(\mathcal{X}_{n}^{\mathbb{Z} \times\{0\}}(t) \cap([a, b] \times\{t\})\right)\right] \leq \frac{C_{29}(b-a)}{\sqrt{t}}
$$

where $C_{29}$ is a constant, independent of t and $a, b$.

Proof : Fix $s>0$ and let $u(s)=\mathbb{E}\left[\#\left(\mathcal{X}^{\mathbb{Z} \times\{0\}}(s) \cap([0,1) \times\{s\})\right)\right]$. Set $M \geq 1$. Then by translation invariance, we have $\mathbb{E}\left[\#\left(\mathcal{X}^{\mathbb{Z} \times\{0\}}(s) \cap([0, M) \times\{s\})\right)\right]=$ 
$M u(s)$. Now, we have

$$
\begin{aligned}
M u(s) & \leq \sum_{i=-\infty}^{\infty} \mathbb{E}\left[\#\left(\mathcal{X}^{[i M,(i+1) M) \times\{0\}}(s) \cap([0, M) \times\{s\})\right)\right] \\
& =\sum_{i=-\infty}^{\infty} \mathbb{E}\left[\#\left(\mathcal{X}^{[0, M) \times\{0\}}(s) \cap([-i M,-(i-1) M)) \times\{s\}\right)\right] \\
& =\mathbb{E}\left[\#\left(\mathcal{X}^{[0, M) \times\{0\}}(s)\right)\right] \\
& \leq M-(M-1)\left[1-\mathbb{P}\left(\pi^{(0,0)}(s) \neq \pi^{(1,0)}(s)\right)\right] \\
& \leq M-(M-1)\left[1-\frac{C_{27}}{\sqrt{s}}\right] \quad(\text { from Proposition [5.9) }) \\
& \leq 1+\frac{C_{27}(M-1)}{\sqrt{s}} .
\end{aligned}
$$

Dividing both sides by $M$ and letting $M \rightarrow \infty$, we have $u(s) \leq C_{27} / \sqrt{s}$.

For any $n \geq 1$, let $l_{n}=\lfloor n(b-a) / 2\rfloor+1$. Now, we have $\mathbb{E}\left[\#\left(\mathcal{X}_{n}^{\mathbb{Z} \times\{0\}}(t) \cap([a, b] \times\right.\right.$ $\{t\}))]=\mathbb{E}\left[\#\left(\mathcal{X}_{n}^{\mathbb{Z} \times\{0\}}(t) \cap([-(b-a) / 2,(b-a) / 2] \times\{t\})\right)\right] \leq \mathbb{E}\left[\#\left(\mathcal{X}^{\mathbb{Z} \times\{0\}}\left(n^{2} \gamma t\right) \cap\right.\right.$ $\left.\left.\left(\left[-l_{n}, l_{n}\right) \times\left\{n^{2} \gamma t\right\}\right)\right)\right]=2 l_{n} u\left(n^{2} \gamma t\right) \leq 2 C_{27} l_{n} / \sqrt{n^{2} \gamma t} \leq C_{29}(b-a) / \sqrt{t}$ for a proper choice of $C_{29}$.

Let $\left(\mathcal{P}, \rho_{\mathcal{P}}\right)$ be the space of compact subsets of $\left(\mathbb{R}_{c}^{2}, \rho\right)$ with the induced Hausdorff metric. Since $\overline{\mathcal{X}}_{n}^{t_{0}}$ converges weakly to $\mathcal{Z}^{t_{0}}$, by the continuous mapping theorem, we have that $\overline{\mathcal{X}}_{n}^{t_{0}}\left(t_{0}+t\right)$ converges weakly to $\mathcal{Z}^{t_{0}}\left(t_{0}+t\right)$ in $\left(\mathcal{P}, \rho_{\mathcal{P}}\right)$. Next we prove that $\mathcal{Z}^{t_{0}}\left(t_{0}+t\right)$ is a.s. locally finite.

Proposition 5.11. For any $t>0, \mathcal{Z}^{t_{0}}\left(t_{0}+t\right)$ is a.s. locally finite and

$$
\mathbb{E}\left[\#\left(\mathcal{Z}^{t_{0}}\left(t_{0}+t\right) \cap\left((a, b) \times\left\{t_{0}+t\right\}\right)\right)\right] \leq \frac{C_{29}(b-a)}{\sqrt{t}}
$$

for $C_{29}$ as in the previous proposition.

Proof : For the first part it is enough to consider $t_{0}=0$ and prove that $\#\left(\mathcal{Z}^{0}(t) \cap\right.$ $((-m, m) \times\{t\}))$ is finite a.s. for any $m \geq 1$. First, we observe that if $\mathbf{u}=$ $(u(1), u(2))$ is such that $u(2)<0$ and $h(\mathbf{u})(2)>0$, then it must be the case that $h(u(1), 0)=h(\mathbf{u})$. Therefore, $\#\left(\overline{\mathcal{X}}_{n}^{0}(t) \cap((-m, m) \times\{t\})\right) \leq \#\left(\mathcal{X}_{n}^{\mathbb{Z} \times\{0\}}(t) \cap\right.$ $((-m, m) \times\{t\}))$. From Proposition 5.10 ,

$$
\begin{aligned}
& \mathbb{E}\left[\liminf _{n \rightarrow \infty} \#\left(\overline{\mathcal{X}}_{n}^{0}(t) \cap((-m, m) \times\{t\})\right)\right] \\
& \leq \mathbb{E}\left[\liminf _{n \rightarrow \infty} \#\left(\mathcal{X}_{n}^{\mathbb{Z} \times\{0\}}(t) \cap((-m, m) \times\{t\})\right)\right] \\
& \leq \liminf _{n \rightarrow \infty} \mathbb{E}\left[\#\left(\mathcal{X}_{n}^{\mathbb{Z} \times\{0\}}(t) \cap((-m, m) \times\{t\})\right)\right] \\
& \leq 2 C_{29} m / \sqrt{t} .
\end{aligned}
$$


Therefore, we conclude that $\liminf _{n \rightarrow \infty} \#\left(\overline{\mathcal{X}}_{n}^{0}(t) \cap((-m, m) \times\{t\})\right)<\infty$ almost surely.

Since $\overline{\mathcal{X}}_{n}^{0}(t) \Rightarrow \mathcal{Z}^{0}(t)$ in $\left(\mathcal{P}, \rho_{\mathcal{P}}\right)$, using Skorohod's representation theorem, we may couple the processes so that the above convergence is almost sure. We now claim that, almost surely,

$$
\#\left(\mathcal{Z}^{0}(t) \cap((-m, m) \times\{t\})\right) \leq \liminf _{n \rightarrow \infty} \#\left(\overline{\mathcal{X}}_{n}^{0}(t) \cap((-m, m) \times\{t\})\right)
$$

Fix $\omega$ such that $\liminf _{n \rightarrow \infty} \#\left(\overline{\mathcal{X}}_{n}^{0}(t) \cap((-m, m) \times\{t\})\right)=l(\omega)<\infty$. For this $\omega$ we may choose a subsequence $n_{k}$ along which $\#\left(\overline{\mathcal{X}}_{n_{k}}^{0}(t) \cap((-m, m) \times\{t\})\right)=l(\omega)$ for all large $k$. If $\mathcal{Z}^{0}(t) \cap((-m, m) \times\{t\})$ has at least $l(\omega)+1$ distinct points, say, $\left\{\left(y_{i}, t\right): i=1, \ldots, l(\omega)+1\right\}$, we may choose $\delta>0$ so small that the intervals $\left(y_{i}-\delta, y_{i}+\delta\right) \subseteq(-m, m)$, for $i=1,2, \ldots, l(\omega)+1$ are mutually disjoint. Since $\overline{\mathcal{X}}_{n_{k}}^{0}(t) \cap([-m, m] \times\{t\})$ converges to $\mathcal{Z}^{0}(t) \cap([-m, m] \times\{t\})$ in $\left(\mathcal{P}, \rho_{\mathcal{P}}\right)$, each of these intervals should contain at least one point of $\overline{\mathcal{X}}_{n_{k}}^{0}(t) \cap((-m, m) \times\{t\})$ for all large $k$. This is a contradiction as for all large $k, \overline{\mathcal{X}}_{n_{k}}^{0}(t) \cap((-m, m) \times\{t\})$ has exactly $l(\omega)$ many points.

The expectation bound now follows from the above inequality and completes the proof.

Since $\mathbb{E}\left[\#\left(\mathcal{Z}^{t_{0}}\left(t_{0}+t\right) \cap\left((x-\delta, x+\delta) \times\left\{t_{0}+t\right\}\right)\right)\right] \leq 2 C_{29} \delta / \sqrt{t} \rightarrow 0$, as $\delta \rightarrow 0$, we may conclude

Corollary 5.11.1. For any $x \in \mathbb{R}, \mathbb{P}\left(\left(x, t_{0}+t\right) \in \mathcal{Z}^{t_{0}}\left(t_{0}+t\right)\right)=0$.

We now state the main proposition of this subsection which is similar to Lemma 6.3 of Newman et al. [17].

Proposition 5.12. For $t>0$, we have

$$
\mathcal{Z}^{t_{0} ;\left(t_{0}+t\right)_{T}} \stackrel{d}{=} \mathcal{W}^{\mathcal{Z}^{t_{0}}\left(t_{0}+t\right)}
$$

where $\mathcal{W}^{\mathcal{Z}^{t_{0}}\left(t_{0}+t\right)}$ is the set of paths given by coalescing Brownian motions starting from a random point set distributed as $\mathcal{Z}^{t_{0}}\left(t_{0}+t\right)$.

We first complete the proof of $\left(E_{1}^{\prime}\right)$ assuming the validity of the above proposition. For $0<\nu<t$, we have,

$$
\begin{aligned}
\mathbb{E}\left[\hat{\eta}_{\mathcal{Z}^{t_{0}}}\left(t_{0}, t ; a, b\right)\right] & =\mathbb{E}\left[\hat{\eta}_{\mathcal{Z}^{t_{0} ;\left(t_{0}+\nu\right)_{T}}}\left(t_{0}+\nu, t-\nu ; a, b\right)\right] \\
& \leq \mathbb{E}\left[\hat{\eta}_{\mathcal{W}}\left(t_{0}+\nu, t-\nu ; a, b\right)\right]=\frac{b-a}{\sqrt{\pi(t-\nu)}}
\end{aligned}
$$

Letting $\nu \rightarrow 0$ we obtain $\left(E_{1}^{\prime}\right)$. 
Before proving Proposition 5.12 we observe that the paths in $\mathcal{X}_{n}^{0 ;(t)_{T}}$ carry their own history region along with them. Hence Proposition 5.6 cannot be applied directly to obtain the finite dimensional distributions of $\mathcal{Z}^{0 ;(t)_{T}}$.

Proof of Proposition 5.12: It suffices to prove the result for $t_{0}=0$. We restrict our attention to the set of paths which start in $[-m, m] \times\{t\}$ for some $m \geq 1$. Let us denote by $\mathcal{Z}_{m}^{0 ;(t)_{T}}=\left\{\pi \in \mathcal{Z}^{0 ;(t)_{T}}: \pi(t) \in[-m, m]\right\}$ and $\mathcal{W}^{\mathcal{Z}_{m}^{t}}=$ $\mathcal{W}^{\mathcal{Z}^{0}(t) \cap([-m, m] \times\{t\})}$. Observe that it is enough to show $\mathcal{Z}_{m}^{0 ;(t)_{T}} \stackrel{d}{=} \mathcal{W}^{\mathcal{Z}_{m}^{t}}$ for any $m \geq 1$. For the rest of the section we fix $m \geq 1$.

Consider the mapping $g: \mathcal{H} \rightarrow \mathcal{H}$ given by $g(K)=\left\{\pi \in K^{0 ;(t)_{T}}: \pi(t) \in\right.$ $[-m, m]\}$. Using Corollary 5.11 .1 we have $\mathbb{P}\left(\mathcal{Z}^{0} \in D_{g}\right)=0$ where $D_{g}$ is the set of discontinuity points of the map $g$. Since $\overline{\mathcal{X}}_{n}^{0} \Rightarrow \mathcal{Z}^{0}$, from Theorem 5.1 of Billingsley [6] we have $g\left(\overline{\mathcal{X}}_{n}^{0}\right) \Rightarrow g\left(\mathcal{Z}^{0}\right)=\mathcal{Z}_{m}^{0 ;(t)_{T}}$.

Now we will obtain $\mathcal{W}^{\mathcal{Z}_{m}^{t}}$ as a weak limit of $g\left(\overline{\mathcal{X}}_{n}^{0}\right)$ to establish the required equality. For any $K \in \mathcal{H}$, we consider the map $f: \mathcal{H} \rightarrow \mathcal{P}$ given by $f(K)=$ $\left\{(\pi(t), t): \sigma_{\pi} \leq t, \pi(t) \in[-m, m]\right\}$. Again, using Corollary [5.11.1, we observe that $\mathbb{P}\left(\mathcal{Z}^{0} \in D_{f}\right)=0$ where $D_{f}$ is the set of all discontinuity points of the map. For $t>0$, taking $D:=\mathcal{Z}^{0}(t) \cap([-m, m] \times\{t\})$, we have that, as $n \rightarrow \infty$,

$$
\overline{\mathcal{X}}_{n}^{0}(t) \cap([-m, m] \times\{t\}) \Rightarrow D \text { in }\left(\mathcal{P}, \rho_{\mathcal{P}}\right) .
$$

Fix $n \geq 1, \beta<1 / 2$ and $2 \beta<\delta<1$. Define the sets

$$
D_{n}:=\left\{\left(\lfloor n \sigma \mathbf{x}(1)\rfloor,\left\lfloor n^{2} \gamma t\right\rfloor+\left\lfloor n^{\beta}\right\rfloor+1\right):(\mathbf{x}(1), \mathbf{x}(2)) \in \mathcal{X}_{n}^{0}(t), \mathbf{x}(1) \in[-m, m]\right\}
$$

and $D_{n}^{\text {(scaled) }}:=\left\{\left(\mathbf{y}(1) /(n \sigma), \mathbf{y}(2) /\left(n^{2} \gamma\right)\right): \mathbf{y} \in D_{n}\right\}$. Note that $D_{n}$ (and hence $D_{n}^{\text {(scaled) })}$ is a finite set. For each $\mathbf{y}=(\mathbf{y}(1), \mathbf{y}(2)) \in D_{n}^{\text {(scaled) }}$, we have $\mathbf{x} \in$ $\mathcal{X}_{n}^{0}(t) \cap([-m, m] \times\{t\})$ such that $\|\mathbf{x}-\mathbf{y}\|_{2} \leq\left(n^{\beta}+2\right) / n$ and vice versa. Thus, $\rho_{\mathcal{P}}\left(\mathcal{X}_{n}^{0}(t) \cap([-m, m] \times\{t\}), D_{n}^{\text {(scaled) }}\right) \rightarrow 0$ almost surely. Using (42) , we conclude that $D_{n}^{\text {(scaled) }} \Rightarrow \mathcal{Z}^{0}(t) \cap([-m, m] \times\{t\})=D$ in $\left(\mathcal{P}, \rho_{\mathcal{P}}\right)$.

We show now that it is unlikely that a path $\pi \in \mathcal{X}_{n}^{0}$, which crosses the x-axis far from the origin, will cross the line $\{y=t\}$ inside $[-m, m]$. Consider the event

$$
E_{n}:=\left\{\text { there exists } \pi \in \mathcal{X}_{n}^{0} \text { with } \pi(0) \notin[-n, n] \text { and } \pi(t) \in[-m, m]\right\} .
$$

Scaling back to the original lattice and using the non-crossing property of paths, we observe that the if the paths starting from $\mathbf{u}=\left(-\left\lfloor n^{2} \sigma\right\rfloor, 0\right)$ and $\mathbf{v}=\left(\left\lfloor n^{2} \sigma\right\rfloor, 0\right)$ do not cross the segment $[-n \sigma m, n \sigma m] \times\left\{n^{2} \gamma t\right\}$, then the paths which cross the $\mathrm{x}$-axis to the left of $-n^{2} \sigma$ or to the right of $n^{2} \sigma$ will also stay away from that 
segment. Hence, we have

$$
\begin{aligned}
\mathbb{P}\left(E_{n}\right) & \leq \mathbb{P}\left(\left\{\pi^{\mathbf{u}}\left(n^{2} \gamma t\right) \geq-n \sigma m\right\} \cup\left\{\pi^{\mathbf{v}}\left(n^{2} \gamma t\right) \leq n \sigma m\right\}\right) \\
& \leq 2 \mathbb{P}\left(\pi^{\mathbf{u}}\left(n^{2} \gamma t\right) \geq-n \sigma m\right) \\
& =2 \mathbb{P}\left(\pi^{\mathbf{0}}\left(n^{2} \gamma t\right) \geq\left\lfloor n^{2} \sigma\right\rfloor-n \sigma m\right) \\
& =2 \mathbb{P}\left(\pi_{n}^{\mathbf{0}}(t) \geq\left(\left\lfloor n^{2} \sigma\right\rfloor-n \sigma m\right) /(n \sigma)\right) \\
& \rightarrow 0 \text { as } n \rightarrow \infty
\end{aligned}
$$

$\pi^{\mathbf{0}}$ being the path starting at $(0,0)$, and the last step follows from Proposition 5.2 .

Let $\tilde{\mathcal{X}}_{n}:=\left\{\pi \in \overline{\mathcal{X}}_{n}^{0}: \pi(0) \in[-n, n]\right\}$. On the event $E_{n}^{c}$, we observe that $g\left(\tilde{\mathcal{X}}_{n}\right)=g\left(\overline{\mathcal{X}}_{n}^{0}\right)$ as $\left(\mathcal{H}, d_{\mathcal{H}}\right)$-valued random variable. Hence, we have

$$
d_{\mathcal{H}}\left(g\left(\tilde{\mathcal{X}}_{n}\right), g\left(\overline{\mathcal{X}}_{n}^{0}\right)\right) \stackrel{\mathbb{P}}{\rightarrow} 0
$$

Now, we follow the paths in $g\left(\tilde{\mathcal{X}}_{n}\right)$ until they cross the line $\{y=t\}$ and consider the history that is created in doing so. Scaling back to the original lattice, for $\mathbf{u} \in \mathbb{Z}^{2}$ with $\mathbf{u}(2) \leq 0$ let $l^{\mathbf{u}}:=\min \left\{j: h^{j}(\mathbf{u})(2) \geq n^{2} \gamma t\right\}$ denote the number of steps taken by the path starting from $\mathbf{u}$ to cross the line $\left\{y=n^{2} \gamma t\right\}$. We define, the set of explored regions for paths in unscaled version of $g\left(\tilde{\mathcal{X}}_{n}\right)$, by

$$
\tilde{\Delta}_{n}:=\bigcup \bigcup_{i=0}^{l^{\mathbf{u}}-1} S^{+}\left(h^{i}(\mathbf{u}),\left\|h^{i}(\mathbf{u})-h^{i+1}(\mathbf{u})\right\|_{1}\right)
$$

where the first union is over $\mathbf{u} \in V, \mathbf{u}(2) \leq 0, \pi_{n}^{\mathbf{u}}(0) \in[-n, n], \pi_{n}^{\mathbf{u}}(t) \in[-m, m]$. Consider the event $F_{n}=\left\{\tilde{\Delta}_{n} \nsubseteq \mathbb{H}\left(\left\lfloor n^{2} \gamma t\right\rfloor+\left\lfloor n^{\beta}\right\rfloor\right)\right\}$. Assuming $\mathbb{P}\left(F_{n}\right) \rightarrow 0$ as $n \rightarrow \infty$ (which will be shown shortly), we observe that on the event $\left(E_{n} \cup F_{n}\right)^{c}$, using the fact that the vertices of $D_{n}$ lie on the line $\left\{y=\left\lfloor n^{2} \gamma t\right\rfloor+\left\lfloor n^{\beta}\right\rfloor+1\right\}$, the evolution of the paths from the set $D_{n}$ is independent of the history $\tilde{\Delta}_{n}$. This allows us to adapt Lemma 6.5 of [17] for our model and conclude

$$
\mathcal{X}_{n}^{D_{n}} \Rightarrow \mathcal{W}^{D}=\mathcal{W}^{\mathcal{Z}_{m}^{t}} \text { in }\left(\mathcal{H}, d_{\mathcal{H}}\right)
$$

The details of this argument is presented in the appendix.

To show $\mathbb{P}\left(F_{n}\right) \rightarrow 0$, we consider paths starting from each $(j, 0),-n^{2} \sigma-1 \leq$ $j \leq n^{2} \sigma+1$ and consider the region explored by these paths until they cross the line $\left\{y=n^{2} \gamma t\right\}$, i.e., set

$$
\Delta_{n}^{\prime}:=\bigcup_{j=-\left\lfloor n^{2} \sigma\right\rfloor-1}^{\left\lfloor n^{2} \sigma\right\rfloor+1} \bigcup_{i=0}^{l^{(j, 0)}-1} S^{+}\left(h^{i}(j, 0),\left\|h^{i}(j, 0)-h^{i+1}(j, 0)\right\|_{1}\right) .
$$


We observe that on the event $E_{n}^{c}$, we must have $\tilde{\Delta}_{n} \backslash \mathbb{H}(0) \subseteq \Delta_{n}^{\prime}$. Therefore, we have

$$
\begin{aligned}
& \mathbb{P}\left(\tilde{\Delta}_{n} \nsubseteq \mathbb{H}\left(\left\lfloor n^{2} \gamma t\right\rfloor+\left\lfloor n^{\beta}\right\rfloor\right)\right) \\
& \leq \mathbb{P}\left(\left\{\Delta_{n}^{\prime} \nsubseteq \mathbb{H}\left(\left\lfloor n^{2} \gamma t\right\rfloor+\left\lfloor n^{\beta}\right\rfloor\right)\right\} \cap E_{n}^{c}\right)+\mathbb{P}\left(E_{n}\right) \\
& \leq \mathbb{P}\left[\bigcup_{j=-\left\lfloor n^{2} \sigma\right\rfloor-1}^{+\left\lfloor n^{2} \sigma\right\rfloor+1} \bigcup_{i=1}^{\left\lfloor n^{2} \gamma t\right\rfloor+1}\left\{W_{i}(j, 0) \geq n^{\beta}\right\}\right]+\mathbb{P}\left(E_{n}\right) \\
& \leq\left(2\left\lfloor n^{2} \sigma\right\rfloor+3\right)\left(\left\lfloor n^{2} \gamma t\right\rfloor+1\right) \mathbb{P}\left(W_{1}(0,0) \geq n^{\beta}\right)+\mathbb{P}\left(E_{n}\right) \\
& \rightarrow 0 \text { as } n \rightarrow \infty,
\end{aligned}
$$

where the penultimate inequality follows from the fact that each path $\pi^{(j, 0)}$ can have at most $\left\lfloor n^{2} \gamma t\right\rfloor+1$ many regenerations until it crosses the line $\left\{y=n^{2} \gamma t\right\}$ and the last step follows from Proposition 2.8.

Finally, we show that $d_{\mathcal{H}}\left(\mathcal{X}_{n}^{D_{n}}, g\left(\overline{\mathcal{X}}_{n}^{0}\right)\right) \stackrel{\mathbb{P}}{\rightarrow} 0$ to complete the proof of Proposition 5.12, Consider the event that one of the paths in $g\left(\tilde{\mathcal{X}}_{n}\right)$ moves significantly far in a short period after crossing the line $\{y=t\}$. Define the event

$$
\begin{aligned}
& G_{n}:=\left\{\text { there exists } \pi \in g\left(\tilde{\mathcal{X}}_{n}\right) \text { with }|\pi(t)-\pi(s)|>n^{\delta-1} / \sigma\right. \\
& \text { for some } \left.s \in\left[t, t+\left(n^{\beta-2} / \gamma\right)\right]\right\} .
\end{aligned}
$$

We have $\#\left(g\left(\tilde{\mathcal{X}}_{n}\right)\right) \leq \#\left\{\pi(t) \in[-m, m]: \pi \in \mathcal{X}_{n}^{\mathbb{Z} \times\{0\}}\right\}$ and hence, on the event $E_{n}^{c}, \#\left(g\left(\tilde{\mathcal{X}}_{n}\right)\right) \leq 2\left\lfloor n^{2} \sigma\right\rfloor+3$. From Proposition 5.4 it follows that $\mathbb{P}\left(G_{n}\right) \leq \mathbb{P}\left(E_{n}\right)+$ $\mathbb{P}\left(F_{n}\right)+\left(2\left\lfloor n^{2} \sigma\right\rfloor+3\right) \mathbb{P}\left(\sup \left\{\left|\pi^{\mathbf{0}}(s)\right|: 0 \leq s \leq n^{\beta}\right\} \geq n^{\delta} \mid\left\{U_{\mathbf{w}}: \mathbf{w} \in \Delta_{n}^{\prime}\right\} \cap\left(F_{n}\right)^{c}\right) \leq$ $\mathbb{P}\left(E_{n}\right)+\mathbb{P}\left(F_{n}\right)+C_{23} n^{\beta} \exp \left(-C_{24} n^{\beta}\right)\left(2\left\lfloor n^{2} \sigma\right\rfloor+3\right) \rightarrow 0$ as $n \rightarrow \infty$.

The finiteness of $g\left(\overline{\mathcal{X}}_{n}^{0}\right)$ allows us to enumerate the paths in $g\left(\overline{\mathcal{X}}_{n}^{0}\right)$ as $\pi^{1}, \ldots, \pi^{N}$ for some random $N$. Let $\mathbf{x}_{j}:=\left(\left\lfloor n \sigma \pi^{j}(t)\right\rfloor,\left\lfloor n^{2} \gamma t\right\rfloor+\left\lfloor n^{\beta}\right\rfloor+1\right)$ for $j=1, \ldots, N$. Note that $\mathbf{x}_{j}$ 's need not be distinct, however $D_{n}=\left\{\mathbf{x}_{i}: 1 \leq i \leq N\right\}$ and hence $d_{\mathcal{H}}\left(\mathcal{X}_{n}^{D_{n}}, g\left(\overline{\mathcal{X}}_{n}^{0}\right)\right) \leq \max \left\{d_{\Pi}\left(\pi^{j}, \pi_{n}^{\mathbf{x}_{j}}\right): 1 \leq j \leq N\right\}$. In other words, for $s>t$ taking $M_{n}^{j}(s):=\sup \left\{\left|\pi^{j}(l)-\pi_{n}^{\mathbf{x}_{j}}(l)\right|: l \in\left[t+\left(\left\lfloor n^{\beta}\right\rfloor+1\right) /\left(n^{2} \gamma\right), s\right]\right\}$ we need to show (a) $\max \left\{M_{n}^{j}(s): 1 \leq j \leq N\right\} \stackrel{\mathbb{P}}{\rightarrow} 0$ and $(\mathrm{b}) \max \left\{\left\|\left(\pi^{j}\left(\sigma_{\pi^{j}}\right), \sigma_{\pi_{j}}\right)-\left(\pi_{n}^{\mathbf{x}_{j}}\left(\sigma_{\pi_{n}} \mathbf{x}_{j}\right), \sigma_{\pi_{n}} \mathbf{x}_{j}\right)\right\|_{2}:\right.$ $1 \leq j \leq N\} \stackrel{\mathbb{P}}{\rightarrow} 0$ as $n \rightarrow \infty$

Since $\left\|\left(\pi^{j}\left(\sigma_{\pi^{j}}\right), \sigma_{\pi_{j}}\right)-\left(\pi_{n}^{\mathbf{x}_{j}}\left(\sigma_{\pi_{n} \mathbf{x}_{j}}\right), \sigma_{\pi_{n} \mathbf{x}_{j}}\right)\right\|_{2} \leq\left(n^{\beta}+2\right) / n$, (b) follows immediately.

Clearly, $\mathbb{E}(N)=\mathbb{E}\left[\#\left(g\left(\overline{\mathcal{X}}_{n}^{0}\right)\right)\right] \leq \mathbb{E}\left[\#\left(\mathcal{X}_{n}^{\mathbb{Z} \times\{0\}}(t) \cap([-m, m] \times\{t\})\right)\right] \leq 2 C_{29} m / t$ from Proposition 5.10, So, given $\eta, \eta^{\prime}>0$, we can choose $L$ (independent of $n$ ) so large that $\mathbb{P}(N \geq L) \leq \eta^{\prime} / 2$. On the event $G_{n}^{c}$, we observe that the paths $\pi^{j}$ and $\pi_{n}^{\mathbf{x}_{j}}$ will lie between the scaled paths starting from $\left(\left\lfloor n \sigma \pi^{j}(t)\right\rfloor-\left\lfloor n^{\delta}\right\rfloor,\left\lfloor n^{2} \gamma t\right\rfloor+\left\lfloor n^{\beta}\right\rfloor+1\right)$ and $\left(\left\lfloor n \sigma \pi^{j}(t)\right\rfloor+\left\lfloor n^{\delta}\right\rfloor,\left\lfloor n^{2} \gamma t\right\rfloor+\left\lfloor n^{\beta}\right\rfloor+1\right)$. Therefore, by translation invariance, $\mathbb{P}\left(\left\{M_{j}(s) \geq \eta\right\} \cap F_{n}^{c}\right) \leq \mathbb{P}\left(\sup \left\{\left|\pi_{n}^{\mathbf{0}}(l)-\pi_{n}^{\left(2\left\lfloor n^{\delta}\right\rfloor, 0\right)}(l)\right|: 0 \leq l \leq s-t\right\} \geq \eta\right)$. Hence, 
we have that $\mathbb{P}\left(\max \left\{M_{n}^{j}(s): 1 \leq j \leq N\right\} \geq \eta\right) \leq \mathbb{P}\left(F_{n}\right)+\mathbb{P}\left(G_{n}\right)+\mathbb{P}\left[\#\left(g\left(\overline{\mathcal{X}}_{n}^{0}\right)\right) \geq\right.$ $L]+L \mathbb{P}\left(\sup \left\{\left|\pi_{n}^{\mathbf{0}}(l)-\pi_{n}^{\left(2\left\lfloor n^{\delta}\right\rfloor, 0\right)}(l)\right|: 0 \leq l \leq s-t\right\} \geq \eta\right)$. By Proposition 5.3, both the paths $\pi_{n}^{\mathbf{0}}$ and $\pi_{n}^{\left(2\left\lfloor n^{\delta}\right\rfloor, 0\right)}$ converge to the same Brownian motion. Therefore, for all large $n$, we have $\mathbb{P}\left(\max \left\{M_{n}^{j}(s): 1 \leq j \leq N\right\} \geq \eta\right)<\eta^{\prime}$. This completes the proof.

\section{Appendix}

Proof of Lemma 2.6: It suffices to prove that, for some $\alpha>0$, we have $\mathbb{E}\left(\exp \left(\alpha \tau_{M}\right)\right)<\infty$. Since $M_{n+1}$ is a function of $M_{n}$ and an independent sequence of random variables, $\left\{M_{n}: n \geq 0\right\}$ is a Markov chain. Furthermore, it is irreducible and recurrent. Using Proposition 5.5, Chapter 1 of Asmussen [3], it suffices to show that there exist a nonnegative function $f: \mathbb{N} \cup\{0\} \rightarrow \mathbb{R}^{+}, n_{0} \in \mathbb{N}$ and $r>1$ such that $f(j)>\nu$ for some $\nu>0$ and $\mathbb{E}\left[f\left(M_{1}\right) \mid M_{0}=j\right]<\infty$ for all $j \leq n_{0}$, while for $j>n_{0}, \mathbb{E}\left[f\left(M_{1}\right) \mid M_{0}=j\right] \leq f(j) / r$.

Taking $f:\{0,1,2, \ldots\} \rightarrow \mathbb{R}$ to be $f(i)=\exp (\alpha i)$, where $\alpha>0$ is small enough so that $\mathbb{E}\left[\exp \left(\alpha \theta_{1}\right)\right]<\infty$ and $\exp (-\alpha)<1 / r$, we have

$$
\begin{aligned}
& \mathbb{E}\left[\exp \left(\alpha\left(M_{1}-M_{0}\right)\right) \mid M_{0}=m\right] \\
& =\exp (-\alpha) \mathbb{P}\left(\theta_{1} \leq m\right)+\exp (-\alpha(m+1)) \mathbb{E}\left[\mathbf{1}\left(\theta_{1}>m\right) \exp \left(\alpha \theta_{1}\right)\right] \\
& <(1 / r)+\exp (-\alpha(m+1)) \mathbb{E}\left[\mathbf{1}\left(\theta_{1}>m\right) \exp \left(\alpha \theta_{1}\right)\right] \\
& \leq(1 / r) \text { for } m \text { sufficiently large. }
\end{aligned}
$$

Here the last inequality follows because $\mathbb{E}\left[\exp \left(\alpha \theta_{1}\right)\right]<\infty$ guarantees $\exp (-\alpha(m+$ 1)) $\mathbb{E}\left[\mathbf{1}\left(\theta_{1}>m\right) \exp \left(\alpha \theta_{1}\right)\right] \rightarrow 0$ as $m \rightarrow \infty$.

Proof of Lemma 2.7; We have $\mathbb{P}(N=n) \leq \mathbb{P}(N \geq n) \leq \mathbb{E}(\alpha N) \exp (-\alpha n)$. Let $\Psi$ be the moment generating function of $\theta_{1}$. Then, for all $\gamma_{0} \leq \beta, \Psi\left(\gamma_{0}\right)=$ $\mathbb{E}\left(\exp \left(\gamma_{0} \theta_{1}\right)\right)<\infty$. Since the function $\Psi\left(\gamma_{0}\right)$ is continuous at 0 and $\Psi(0)=1$, we may choose $\gamma>0$ so that $\Psi(2 \gamma) \exp (-\alpha)<1$. Now, we have

$$
\begin{aligned}
& \mathbb{E}[\exp (\gamma S)]=\mathbb{E}\left[\sum_{n=1}^{\infty} \mathbf{1}(N=n) \exp \left(\gamma \sum_{i=1}^{n} \theta_{i}\right)\right] \\
& =\sum_{n=1}^{\infty} \mathbb{E}\left[\mathbf{1}(N=n) \exp \left(\gamma \sum_{i=1}^{n} \theta_{i}\right)\right] \leq \sum_{n=1}^{\infty}[\mathbb{P}(N=n)]^{1 / 2}\left[\mathbb{E}\left(\exp \left(2 \gamma \sum_{i=1}^{n} \theta_{i}\right)\right)\right]^{1 / 2} \\
& \leq \sum_{n=1}^{\infty} \sqrt{\mathbb{E}(\alpha N)} \exp (-n \alpha / 2)[\Psi(2 \gamma)]^{n / 2}=\sqrt{\mathbb{E}(\alpha N)} \sum_{n=1}^{\infty}[\exp (-\alpha) \Psi(2 \gamma)]^{n / 2}<\infty
\end{aligned}
$$


here the first inequality follows from the Cauchy-Schwartz inequality. This completes the proof.

Proof of Lemma 3.2 : Define $L_{n}:=\max \left\{R_{n}^{(1)}, R_{n}^{(2)}\right\}$ and set $\tau^{L}:=\inf \{n \geq$ $\left.1: L_{n}=0\right\}$. Then, we have $\tau^{R}=\tau^{L}$. Again, we define a new Markov chain which dominates $L_{n}$ and satisfies the conditions of Lemma 2.6, from which we will conclude the result.

We start with 2 families of independent copies of the inter-arrival times, say $\left\{\eta_{n}^{(1)}: n \geq 1\right\}$ and $\left\{\eta_{n}^{(2)}: n \geq 1\right\}$ with $\eta_{1}^{(i)} \stackrel{d}{=} \xi_{1}^{(i)}$ for $i=1,2$. Now keeping the same notation as in the proof of Proposition 2.3, we set $W_{n}^{\text {move }}:=\left\{i: R_{n}^{(i)}=0\right.$ for $i=1,2\}$ and $W_{n}^{\text {stay }}:=\{1,2\} \backslash W_{n}^{\text {move }}$. Now, for $i \in W_{n}^{\text {move }}$, we have $S_{l_{i}(n)}^{(i)}=n$ for some $l_{i}(n) \geq 0$, and, for $i \in W_{n}^{\text {stay }}$, we have $S_{l}^{(i)} \neq n$ for every $l \geq 0$. Define

$$
J_{n+1}:=\max \left\{\max \left\{\xi_{l_{i}(n)+1}^{(i)}: i \in W_{n}^{\text {move }}\right\}, \max \left\{\eta_{n+1}^{(i)}: i \in W_{n}^{\text {stay }}\right\}\right\}
$$

and

$$
M_{0}:=0 \text { and } M_{n+1}:=\max \left\{M_{n}, J_{n+1}\right\}-1 \text { for } n \geq 0 .
$$

We now claim $M_{n} \geq L_{n}$ for all $n \geq 0$. We have $M_{0}=L_{0}=0$. Assume that the result holds for $n$ and we have

$$
L_{n+1}=\max \left\{L_{n}, \max \left\{\xi_{l_{i}(n)+1}^{(i)}: i \in W_{n}^{\operatorname{move}}\right\}\right\}-1 \leq \max \left\{M_{n}, J_{n+1}\right\}-1=M_{n+1} .
$$

The assumptions imposed on $\xi_{n}^{(i)}$ imply that the Markov chain satisfies the conditions of Lemma 2.6 and the result follows from that.

Calculations for $(\underline{\mathbf{2 6}}),(\mathbf{2 7})$ and $(\mathbf{2 8})$ : For all $m \geq 1$ we have

$$
\begin{aligned}
\mathbb{E} & {\left[\left(\left\|\left(\overline{\mathbf{u}^{0}}+\psi_{1}^{\mathbf{u}^{0}}\right)-\left(\overline{\mathbf{v}^{0}}+\psi_{1}^{\mathbf{v}^{0}}\right)\right\|_{2}^{2}-\|\mathrm{x}\|_{2}^{2}\right)^{m}\right] } \\
=\mathbb{E} & {\left[\left(\left\|\left(\mathrm{x}(1)+\psi_{1}^{\mathbf{u}^{0}}(1)-\psi_{1}^{\mathbf{v}^{0}}(1), \mathrm{x}(2)+\psi_{1}^{\mathbf{u}^{0}}(2)-\psi_{1}^{\mathbf{v}^{0}}(2)\right)\right\|_{2}^{2}-\|\mathrm{x}\|_{2}^{2}\right)^{m}\right] } \\
=\mathbb{E} & {\left[\left(\left(\psi_{1}^{\mathbf{u}^{0}}(1)\right)^{2}+\left(\psi_{1}^{\mathbf{u}^{0}}(2)\right)^{2}+\left(\psi_{1}^{\mathbf{v}^{0}}(1)\right)^{2}+\left(\psi_{1}^{\mathbf{v}^{0}}(2)\right)^{2}-2 \psi_{1}^{\mathbf{u}^{0}}(1) \psi_{1}^{\mathbf{v}^{0}}(1)\right.\right.} \\
& \left.\left.\quad-2 \psi_{1}^{\mathbf{u}^{0}}(2) \psi_{1}^{\mathbf{v}^{0}}(2)+2 \mathrm{x}(1)\left(\psi_{1}^{\mathbf{u}^{0}}(1)-\psi_{1}^{\mathbf{v}^{0}}(1)\right)+2 \mathrm{x}(2)\left(\psi_{1}^{\mathbf{u}^{0}}(2)-\psi_{1}^{\mathbf{v}^{0}}(2)\right)\right)^{m}\right] .
\end{aligned}
$$

From Proposition 3.4 we have $\mathbb{E}\left[\left(\psi_{1}^{\mathbf{u}^{0}}\left(j_{1}\right)\right)^{m_{1}}\left(\psi_{1}^{\mathbf{v}^{0}}\left(j_{2}\right)\right)^{m_{2}}\right]=0$ at least one of $m_{1}, m_{2}$ is odd. Hence for $m=1$ we have,

$$
\begin{aligned}
& \mathbb{E}\left[\left\|\left(\overline{\mathbf{u}^{0}}+\psi_{1}^{\mathbf{u}^{0}}\right)-\left(\overline{\mathbf{v}^{0}}+\psi_{1}^{\mathbf{v}^{0}}\right)\right\|_{2}^{2}-\|\mathrm{x}\|_{2}^{2}\right] \\
& =\mathbb{E}\left[\left(\psi_{1}^{\mathbf{u}^{0}}(1)\right)^{2}+\left(\psi_{1}^{\mathbf{u}^{0}}(2)\right)^{2}+\left(\psi_{1}^{\mathbf{v}^{0}}(1)\right)^{2}+\left(\psi_{1}^{\mathbf{v}^{0}}(2)\right)^{2}\right]=4 \mathbb{E}\left[\left(\psi_{1}^{\mathbf{u}^{0}}(1)\right)^{2}\right] .
\end{aligned}
$$


For $m=2$ using Proposition 3.4 we have

$$
\begin{aligned}
\mathbb{E} & {\left[\left(\left\|\left(\overline{\mathbf{u}^{0}}+\psi_{1}^{\mathbf{u}^{0}}\right)-\left(\overline{\mathbf{v}^{0}}+\psi_{1}^{\mathbf{v}^{0}}\right)\right\|_{2}^{2}-\|\mathrm{x}\|_{2}^{2}\right)^{2}\right] } \\
= & \mathbb{E}\left[\left(\left(\left(\psi_{1}^{\mathbf{u}^{0}}(1)\right)^{2}+\left(\psi_{1}^{\mathbf{u}^{0}}(2)\right)^{2}+\left(\psi_{1}^{\mathbf{v}^{0}}(2)\right)^{2}+\left(\psi_{1}^{\mathbf{v}^{0}}(2)\right)^{2}\right)-2 \psi_{1}^{\mathbf{u}^{0}}(1) \psi_{1}^{\mathbf{v}^{0}}(1)\right.\right. \\
& \left.\left.\quad-2 \psi_{1}^{\mathbf{u}^{0}}(2) \psi_{1}^{\mathbf{v}^{0}}(2)+2 \mathrm{x}(1)\left(\psi_{1}^{\mathbf{u}^{0}}(1)-\psi_{1}^{\mathbf{v}^{0}}(1)\right)+2 \mathrm{x}(2)\left(\psi_{1}^{\mathbf{u}^{0}}(2)-\psi_{1}^{\mathbf{v}^{0}}(2)\right)\right)^{2}\right] \\
\geq & \mathbb{E}\left[\left(2 \mathrm{x}(1)\left(\psi_{1}^{\mathbf{u}^{0}}(1)-\psi_{1}^{\mathbf{v}^{0}}(1)\right)\right)^{2}+\left(2 \mathrm{x}(2)\left(\psi_{1}^{\mathbf{u}^{0}}(2)-\psi_{1}^{\mathbf{v}^{0}}(2)\right)\right)^{2}\right] \\
= & 4(\mathrm{x}(1))^{2} \mathbb{E}\left[\left(\psi_{1}^{\mathbf{u}^{0}}(1)\right)^{2}+\left(\psi_{1}^{\mathbf{v}^{0}}(1)\right)^{2}\right]+4(\mathrm{x}(2))^{2} \mathbb{E}\left[\left(\psi_{1}^{\mathbf{u}^{0}}(2)\right)^{2}+\left(\psi_{1}^{\mathbf{v}^{0}}(2)\right)^{2}\right] \\
= & 8\|\mathrm{x}\|_{2}^{2} \mathbb{E}\left[\left(\psi_{1}^{\mathbf{u}^{0}}(1)\right)^{2}\right] .
\end{aligned}
$$

The inequality follows from the fact that $\mathbb{E}\left[\left(\psi_{1}^{\mathbf{u}^{0}}\left(j_{1}\right)\right)^{m_{1}}\left(\psi_{1}^{\mathbf{v}^{0}}\left(j_{2}\right)\right)^{m_{2}}\right] \neq 0$ for all $1 \leq j_{1}, j_{2} \leq d-1$, only if both $m_{1}$ and $m_{2}$ are even and $\mathbb{E}\left[\psi_{1}^{\mathbf{u}^{0}}(1) \psi_{1}^{\mathbf{u}^{0}}(2)\right]=$ $\mathbb{E}\left[\psi_{1}^{\mathbf{v}^{0}}(1) \psi_{1}^{\mathbf{v}^{0}}(2)\right]=0$.

By the same logic it also follows that for $m=3$ we have

$$
\begin{aligned}
& \mathbb{E}\left[\left(\left\|\left(\overline{\mathbf{u}^{0}}+\psi_{1}^{\mathbf{u}^{0}}\right)-\left(\overline{\mathbf{v}^{0}}+\psi_{1}^{\mathbf{v}^{0}}\right)\right\|_{2}^{2}-\|\mathbf{x}\|_{2}^{2}\right)^{3}\right] \\
& =\mathbb{E}\left[\left(\left(\left(\psi_{1}^{\mathbf{u}^{0}}(1)\right)^{2}+\left(\psi_{1}^{\mathbf{u}^{0}}(2)\right)^{2}+\left(\psi_{1}^{\mathbf{v}^{0}}(2)\right)^{2}+\left(\psi_{1}^{\mathbf{v}^{0}}(2)\right)^{2}\right)-2 \psi_{1}^{\mathbf{u}^{0}}(1) \psi_{1}^{\mathbf{v}^{0}}(1)\right.\right. \\
& \left.\left.-2 \psi_{1}^{\mathbf{u}^{0}}(2) \psi_{1}^{\mathbf{v}^{0}}(2)+2 \mathrm{x}(1)\left(\psi_{1}^{\mathbf{u}^{0}}(1)-\psi_{1}^{\mathbf{v}^{0}}(1)\right)+2 \mathrm{x}(2)\left(\psi_{1}^{\mathbf{u}^{0}}(2)-\psi_{1}^{\mathbf{v}^{0}}(2)\right)\right)^{3}\right] \\
& =12 \mathbb{E}\left[\left(\left(\psi_{1}^{\mathbf{u}^{0}}(1)\right)^{2}+\left(\psi_{1}^{\mathbf{u}^{0}}(2)\right)^{2}+\left(\psi_{1}^{\mathbf{v}^{0}}(1)\right)^{2}+\left(\psi_{1}^{\mathbf{v}^{0}}(2)\right)^{2}-2\left(\psi_{1}^{\mathbf{u}^{0}}(1) \psi_{1}^{\mathbf{v}^{0}}(1)+\right.\right.\right. \\
& \left.\left.\psi_{1}^{\mathbf{u}^{0}}(2) \psi_{1}^{\mathbf{v}^{0}}(2)\right)\right)\left(\left(\mathrm{x}(1)\left(\psi_{1}^{\mathbf{u}^{0}}(1)-\psi_{1}^{\mathbf{v}^{0}}(1)\right)\right)^{2}+\left(\mathrm{x}(2)\left(\psi_{1}^{\mathbf{u}^{0}}(2)-\psi_{1}^{\mathbf{v}^{0}}(2)\right)\right)^{2}\right)+ \\
& \text { terms free of } \mathrm{x} \text { ] } \\
& =24\|\mathrm{x}\|_{2}^{2} \mathbb{E}\left[\left(\psi_{1}^{\mathbf{u}^{0}}(1)\right)^{4}+\left(\psi_{1}^{\mathbf{u}^{0}}(1) \psi_{1}^{\mathbf{u}^{0}}(2)\right)^{2}+4\left(\psi_{1}^{\mathbf{u}^{0}}(1) \psi_{1}^{\mathbf{v}^{0}}(1)\right)^{2}\right]+\text { terms free of } \mathbf{x} \\
& =O\left(\|\mathrm{x}\|_{2}^{2}\right) \text { as }\|\mathrm{x}\|_{2} \rightarrow \infty \text {. }
\end{aligned}
$$

Proof of Lemma 5.5: Let $i \in\{1, \ldots, k-1\}$ be such that $\pi_{i}\left(t^{k}\right)=\pi_{k}\left(t^{k}\right)$ and $\pi_{j}\left(t^{k}\right) \neq \pi_{k}\left(t^{k}\right)$ for all $1 \leq j<i$. Fix $\epsilon$ such that $0<\epsilon<t^{k}-\max \left\{\sigma_{\pi_{i}}, \sigma_{\pi_{k}}\right\}$. Given $\eta>0$ let $P_{i}, P_{k} \subseteq \mathbb{R}^{2}$ be defined as

$$
\begin{aligned}
& P_{i}(\eta)=\left\{(x, u):\left\|(x, u)-\left(\pi_{i}(s), s\right)\right\|_{1} \leq \eta \text { for some } \sigma_{\pi_{i}} \leq s \leq t^{k}-\epsilon\right\} \\
& P_{k}(\eta)=\left\{(x, u):\left\|(x, u)-\left(\pi_{k}(s), s\right)\right\|_{1} \leq \eta \text { for some } \sigma_{\pi_{k}} \leq s \leq t^{k}-\epsilon\right\},
\end{aligned}
$$

i.e. $P_{i}$ and $P_{k}$ are the regions obtained by $\eta$-fattening the paths $\pi_{i}$ and $\pi_{k}$ respectively. Since $t^{k}=\inf \left\{s: \pi_{i}(s)=\pi_{k}(s)\right\}>\max \left\{\sigma_{\pi_{i}}, \sigma_{\pi_{k}}\right\}$ therefore we may first choose $0<\eta<\epsilon / 2$ such that

$$
d\left(P_{i}(\eta), P_{k}(\eta)\right):=\inf \left\{\|(x, u)-(y, v)\|_{1}:(x, u) \in P_{i}(\eta),(y, v) \in P_{k}(\eta)\right\}>\eta .
$$

Next, since $d_{\Pi}\left(\pi_{i, n}, \pi_{i}\right) \rightarrow 0$ and $d_{\Pi}\left(\pi_{k, n}, \pi_{k}\right) \rightarrow 0$ as $n \rightarrow \infty$ we may choose $n_{0} \geq 1$ such that $\eta>n_{0}^{\alpha-1}$ and, for all $n \geq n_{0}$, the following hold: 
(a) $\sigma_{\pi_{i, n}} \leq t^{k}-\epsilon$ and $\sigma_{\pi_{i, n}} \leq t^{k}-\epsilon$,

(b) $\left\{\left(\pi_{i, n}(s), s\right): \sigma_{\pi_{i, n}} \leq s \leq t^{k}-\epsilon\right\} \subseteq P_{i}(\eta)$ and $\left\{\left(\pi_{k, n}(s), s\right): \sigma_{\pi_{k, n}} \leq s \leq\right.$ $\left.t^{k}-\epsilon\right\} \subseteq P_{k}(\eta)$

Since $P_{i}$ and $P_{k}$ are separated by a minimum distance $\eta$, we have $t_{n}^{k} \geq t^{k}-\epsilon$ for all $n \geq n_{0}$ and hence $\liminf _{n \rightarrow \infty} t_{n}^{k} \geq t^{k}-\epsilon$.

Now assume that $\pi_{k}\left(t^{k}-\epsilon\right)>\pi_{i}\left(t^{k}-\epsilon\right)$. For the other case the argument is exactly similar. Fix $s \in\left[t^{k}, t^{k}+\epsilon\right]$, such that $\pi_{i}(s)-\pi_{k}(s)=\nu>0$. For $n_{0}$ as above, choose $n_{1}>n_{0}$ such that for all $n \geq n_{1}$ we have $\sup _{t \in\left[t^{k}-\epsilon, t^{k}+\epsilon\right]}\left|\pi_{j, n}(t)-\pi_{j}(t)\right|<\nu / 4$ for $j=i, k$. For $n>n_{1}, \pi_{i, n}(s)-\pi_{k, n}(s) \geq \pi_{i}(s)-\pi_{k}(s)-\left|\pi_{i, n}(s)-\pi_{i}(s)\right|-\mid \pi_{k, n}(s)-$ $\pi_{k}(s) \mid>\nu / 2>0$; and our choice of $n_{1}$ ensures that $\pi_{k, n}\left(t^{k}-\epsilon\right)-\pi_{i, n}\left(t^{k}-\epsilon\right)>0$. Thus, $\pi_{i, n}$ and $\pi_{k, n}$ cross each other before time $t^{k}+\epsilon$ and hence $\lim _{\sup _{n \rightarrow \infty}} t_{n}^{k} \leq$ $t^{k}+\epsilon$. This completes the proof of first part of the Lemma.

Since $t_{n}^{k} \rightarrow t^{k}$ as $n \rightarrow \infty, d_{\Pi}\left(\pi_{i}, \pi_{i, n}\right) \rightarrow 0$ and $d_{\Pi}\left(\pi_{k}, \pi_{k, n}\right) \rightarrow 0$ as $n \rightarrow \infty$, to show $d_{\Pi}^{k}\left(f_{n}^{(\alpha)}\left(\pi_{1, n}, \ldots, \pi_{k, n}\right), f\left(\pi_{1}, \ldots, \pi_{k}\right)\right) \rightarrow 0$ as $n \rightarrow \infty$ it suffices to how that $\sup _{t \in\left[t^{k}-\epsilon, t^{k}+\epsilon\right]}\left|\bar{\pi}_{k, n}(t)-\bar{\pi}_{k}(t)\right| \rightarrow 0$ as $n \rightarrow \infty$.

For $0<\beta<\epsilon$, writing

$$
\begin{aligned}
& \sup _{t \in\left[t^{k}-\epsilon, t^{k}+\epsilon\right]}\left|\bar{\pi}_{k, n}(t)-\bar{\pi}_{k}(t)\right| \leq \sup _{t \in\left[t^{k}-\epsilon, t^{k}-\beta\right]}\left|\pi_{k, n}(t)-\pi_{k}(t)\right| \\
& \quad+\sup _{t \in\left[t^{k}-\beta, t^{k}+\beta\right]}\left|\bar{\pi}_{k, n}(t)-\bar{\pi}_{k}(t)\right|+\sup _{t \in\left[t^{k}+\beta, t^{k}+\epsilon\right]}\left|\pi_{i, n}(t)-\pi_{i}(t)\right| .
\end{aligned}
$$

and observing that

(a) the first and the last terms of the expression above can be made arbitrarily small as in the first part of this proof,

(b) the middle term can be made small by choosing $\beta$ such that, for each of $j_{1}, j_{2} \in\{i, k\}, \sup _{s_{1}, s_{2} \in\left[t^{k}-\beta, t^{k}+\beta\right]}\left|\pi_{j_{1}}\left(s_{1}\right)-\pi_{j_{2}}\left(s_{2}\right)\right|$ is small and noting that $\bar{\pi}_{k, n}$ is defined by a linear interpolation between $\pi_{k, n}\left(t_{n}^{k}\right)$ and $\pi_{i, n}\left(\frac{\left\lfloor n^{2} \gamma t_{n}^{k}\right\rfloor+1}{n^{2} \gamma}\right)$.

Finally, to show (44) we first show that for any deterministic finite sets $B_{n}$ and $B$ with $B_{n} \subset \mathbb{Z}^{2}, B \subset \mathbb{R}^{2} \rho_{\mathcal{P}}\left(B_{n}^{\text {(scaled) }}, B\right) \rightarrow 0$ as $n \rightarrow \infty$ where $B_{n}^{\text {(scaled) }:=}$ $\left\{\left(\mathbf{y}(1) /(n \sigma), \mathbf{y}(2) /\left(n^{2} \gamma\right)\right): \mathbf{y} \in B_{n}\right\}$, we have $\mathcal{X}_{n}^{B_{n}}$ converges weakly to $\mathcal{W}^{B}$, i.e., coalescing Brownian motions starting from a random point set distributed as $B$. Since almost surely $\mathcal{X}^{\mathbb{Z}^{2}}$ consists of noncrossing paths only, $\left(I_{1}\right)$ implies that the family $\left\{\overline{\mathcal{X}_{n}^{\mathbb{Z}^{2}}}: n \in \mathbb{N}\right\}$ is tight, and $\mathcal{X}_{n}^{B_{n}} \subset \overline{\mathcal{X}_{n}^{\mathbb{Z}^{2}}}$ guarantees that $\left\{\mathcal{X}_{n}^{B_{n}}: n \in \mathbb{N}\right\}$ is also tight. The sequence $\left\{\overline{\mathcal{X}_{n}^{\mathbb{Z}^{2}}}: n \in \mathbb{N}\right\}$ also satisfies $\left(I_{1}\right)$ and hence satisfies $\left(B_{1}\right)$. The proof of Theorem 5.3 in [1] shows that for any subsequential limit 
$\mathcal{Z}$ of $\left\{\overline{\mathcal{X}_{n}^{\mathbb{Z}^{2}}}: n \in \mathbb{N}\right\}$ and for any deterministic $\mathbf{x} \in \mathbb{R}^{2}$ there is almost surely a unique path starting from $\mathbf{x}$ in $\mathcal{Z}$. A coupling argument then shows that the same is true for any subsequential limit $\mathcal{Z}_{B}$ of $\left\{\mathcal{X}_{n}^{B_{n}}: n \in \mathbb{N}\right\}$. The sequence $\left\{\left(\overline{\mathcal{X}_{n}^{\mathbb{Z}^{2}}}, \mathcal{X}_{n}^{B_{n}}\right): n \in \mathbb{N}\right\}$ is jointly tight and let $\left(\mathcal{Z}, \mathcal{Z}_{B}\right)$ be a subsequential limit of this sequence. By Skorohod's representation theorem we assume that we are working on a probability space such that $\left\{\left(\overline{\mathcal{X}_{n_{k}}^{\mathbb{Z}^{2}}}, \mathcal{X}_{n_{k}}^{B_{n_{k}}}\right): k \in \mathbb{N}\right\}$ converges almost surely to $\left(\mathcal{Z}, \mathcal{Z}_{B}\right)$. Since $\mathcal{X}_{n_{k}}^{B_{n_{k}}} \subseteq \overline{\mathcal{X}_{n_{k}}^{\mathbb{Z}^{2}}}$ for all $n_{k}$, if for any deterministic $\mathbf{x} \in B$, with positive probability $\mathcal{Z}_{B}$ has more than one path starting from $\mathbf{x}$ then so does $\mathcal{Z}$. Hence for all $\mathbf{x} \in B, \mathcal{Z}_{B}$ has unique path starting from $\mathbf{x}$ almost surely. Now by $\left(I_{1}\right)$ the finite dimensional distributions of $\mathcal{Z}_{B}$ are the same as that of a process of a coalescing Brownian motions. Therefore we have that $\mathcal{Z}_{B}$ has the same distribution as $\mathcal{W}^{B}$ starting from the set $B$.

For the general case, it suffices to show that $\mathbb{E}\left[f\left(\mathcal{X}_{n}^{D_{n}}\right)\right] \rightarrow \mathbb{E}\left[f\left(\mathcal{W}^{D}\right)\right]$ as $n \rightarrow$ $\infty$ for all bounded continuous $f$ on $\left(\mathcal{H}, d_{\mathcal{H}}\right)$. Let $f_{n}\left(D_{n}\right):=\mathbb{E}\left[f\left(\mathcal{X}_{n}^{D_{n}}\right) \mid D_{n}\right]$ and $f(D):=\mathbb{E}\left[f\left(\mathcal{W}^{D}\right) \mid D\right]$. By Skorohod's representation theorem we can assume that we are working on a probability space such that $D_{n} \rightarrow D$ almost surely as $n \rightarrow \infty$ in $\left(\mathcal{P}, \rho_{\mathcal{P}}\right)$. Let $\left\{U_{\mathbf{w}}^{a}: \mathbf{w} \in \mathbb{Z}^{2}\right\}$ be a collection of i.i.d. $U[0,1]$ random variables and independent of the collection $\left\{U_{\mathbf{w}}: \mathbf{w} \in \mathbb{Z}^{2}\right\}$ used to build the model. For any $A \subseteq \mathbb{Z}$, let $\mathcal{X}_{a, n}^{A}$ be the collection of scaled paths starting from $A$ constructed using $\left\{U_{\mathbf{w}}^{a}: \mathbf{w} \in \mathbb{Z}^{2}\right\}$ only. Since the evolution of the paths from $D_{n}$ is independent of $\sigma\left(D_{n}\right)$, we have

$$
\chi_{n}^{D_{n}} \mid D_{n} \stackrel{d}{=} \chi_{a, n}^{D_{n}} \text { almost surely. }
$$

From our assumptions on $D_{n}$ we have $f_{n}\left(D_{n}\right):=\mathbb{E}\left[f\left(\mathcal{X}_{n}^{D_{n}}\right) \mid D_{n}\right]=\mathbb{E}\left[f\left(\mathcal{X}_{a, n}^{D_{n}}\right)\right]$ almost surely. Then, for almost every $\omega$, by the deterministic part of this proof we have that $\mathcal{X}_{a, n}^{D_{n}(\omega)}$ converges in distribution to $\mathcal{W}^{D(\omega)}$. Hence we have almost surely $f_{n}\left(D_{n}\right) \rightarrow f(D)$ as $n \rightarrow \infty$. By the bounded convergence theorem we have $\mathbb{E}\left[f_{n}\left(D_{n}\right)\right]=\mathbb{E}\left[f\left(\mathcal{X}_{n}^{D_{n}}\right)\right] \rightarrow \mathbb{E}[f(D)]=\mathbb{E}\left[f\left(\mathcal{W}^{D}\right)\right]$ as $n \rightarrow \infty$

Acknowledgements: We thank the referee for his comments which led to a significant improvement of this paper. Kumarjit Saha is grateful to Indian Statistical Institute for a fellowship to pursue his Ph.D.

\section{References}

[1] R. Arratia. Coalescing Brownian motions on the line. Ph.D. Thesis. University of Wisconsin, Madison, 1979.

[2] R. Arratia. Coalescing Brownian motions and the voter model on $\mathbb{Z}$. Unpublished partial manuscript. 
[3] S. Asmussen. Applied Probability and Queues. Springer-Verlag, New York, 2003.

[4] S. Athreya, R. Roy and A. Sarkar. Random directed trees and forestdrainage networks with dependence. Electron. J. Prob. 13(2008) 2160-2189.

[5] F. Baccelli and C. Bordenave. The radial spanning tree of a Poisson point process. Ann. Appl. Probab. 17(2007) 305-359.

[6] P. Billingsley. Convergence of probability measures. Wiley, New York, 1968.

[7] C.F. Coletti, L.R.G. Fontes and E.S. Dias. Scaling limit for a drainage network model. J. Appl. Probab. 46(2009) 1184-1197.

[8] D. Coupier and V.C. Tran. The Directed Spanning Forest is almost surely a tree. Random Structures and Algorithms 42(2013) 59-72.

[9] R. Durrett. Probability: Theory and Examples. Cambridge Univ. Press, New York, 2010.

[10] P.A. Ferrari, L.R.G. Fontes and X.-Y. Wu. Two-dimensional Poisson Trees converge to the Brownian web. Ann. Inst. H. Poincaré Probab. Statist. 41(2005) 851-858.

[11] L.R.G. Fontes, M. Isopi, C.M. Newman and K. Ravishankar. The Brownian web: characterization and convergence. Ann. Probab. 32(2004) 2857-2883.

[12] P.A. Ferrari, C. Landim and H. Thorisson. Poisson trees, succession lines and coalescing random walks. Ann. Inst. H. Poincaré Probab. Statist. 40(2004) $141-152$.

[13] S. Gangopadhyay, R. Roy and A. Sarkar. Random oriented trees: a model of drainage networks. Ann. Appl. Probab. 14(2004) 1242-1266.

[14] H. Herrlich and G.E. Strecker. Category theory. Allyn and Bacon, Inc. Boston, 1973.

[15] O. Kallenerg. Foundations of modern probability. Springer-Verlag, New York, 2002.

[16] D.A. Levin, Y. Peres and E.L. Wilmer. Markov chains and mixing times. A. M. S., Providence, Rhode Island, 2008.

[17] C.M. Newman, K. Ravishankar and R. Sun. Convergence of coalescing nonsimple random walks to the Brownian web. Electron. J. Prob. 10(2005) 21-60. 
[18] I. Rodriguez-Iturbe and A. Rinaldo. Fractal river basins: chance and selforganization. Cambridge Univ. Press, New York, 1997.

[19] Scheidegger, A. E. A stochastic model for drainage pattern into an intramontane trench. Bull. Ass. Sci. Hydrol. 12(1967) 15-20.

[20] B. Tóth and W. Werner. The true self-repelling motion. Probab. Theory Related Fields 111(1998) 375-452.

Rahul Roy, Kumarjit Saha and Anish Sarkar Theoretical Statistics and Mathematics Unit

Indian Statistical Institute

7 S. J. S. Sansanwal Marg

New Delhi 110016, India.

rahul,kumarjit10r, anish@isid.ac.in 